\title{
Equilibrium beam distribution in an electron storage ring near linear synchrobetatron coupling resonances
}

\author{
Boaz Nash, ${ }^{*}$ Juhao Wu, and Alexander W. Chao \\ SLAC, Menlo Park, California 94025, USA \\ (Received 2 October 2005; published 8 March 2006)
}

\begin{abstract}
Linear dynamics in a storage ring can be described by the one-turn map matrix. In the case of a resonance where two of the eigenvalues of this matrix are degenerate, a coupling perturbation causes a mixing of the uncoupled eigenvectors. A perturbation formalism is developed to find eigenvalues and eigenvectors of the one-turn map near such a linear resonance. Damping and diffusion due to synchrotron radiation can be obtained by integrating their effects over one turn, and the coupled eigenvectors can be used to find the coupled damping and diffusion coefficients. Expressions for the coupled equilibrium emittances and beam distribution moments are then derived. In addition to the conventional instabilities at the sum, integer, and half-integer resonances, it is found that the coupling can cause an instability through antidamping near a sum resonance even when the symplectic dynamics are stable. As one application of this formalism, the case of linear synchrobetatron coupling is analyzed where the coupling is caused by dispersion in the rf cavity, or by a crab cavity. Explicit closed-form expressions for the sum/difference resonances are given along with the integer/half-integer resonances. The integer and half-integer resonances caused by coupling require particular care. We find an example of this with the case of a crab cavity for the integer resonance of the synchrotron tune. Whether or not there is an instability is determined by the value of the horizontal betatron tune, a unique feature of these coupling-caused integer or half-integer resonances. Finally, the coupled damping and diffusion coefficients along with the equilibrium invariants and projected emittances are plotted as a function of the betatron and synchrotron tunes for an example storage ring based on PEP-II.
\end{abstract}

DOI: $10.1103 /$ PhysRevSTAB.9.032801

\section{INTRODUCTION}

The design of an electron storage ring typically uses a lattice of bending and focusing magnets to steer and focus the beam along with rf cavities to replenish energy to the beam lost to radiation. Two important questions to ask about a given storage ring are (1) is the motion of the stored particles stable, and if so (2) what will be the distribution of particles that are stored? When nonlinear effects can be ignored, the answer to the first question can be given quite concisely. One constructs the symplectic one-turn map matrix, and if all the eigenvalues have magnitude 1, the ring is stable, otherwise there is an instability. As for the second question, the stored electron beam will reach a unique distribution determined by a balance between the diffusion and the damping effects of synchrotron radiation [1]. The beam distribution is also affected by other diffusion processes such as intrabeam scattering. In this paper, we consider only radiation effects which are often dominant.

Electron storage rings are often designed so that nominally there is no coupling among the transverse ( $x$ and $y$ ) and the longitudinal $(z)$ dimensions. The real storage ring, however, will always have some coupling: explicitly due to intentional coupling elements, such as a solenoid in a collider interaction region [2], or a crab cavity for crossing

*Electronic address: bnash@slac.stanford.edu
PACS numbers: 29.27.Bd, 29.20.Dh, 29.27.Eg, 41.85.-p

angle compensation [3], and undesirably because of small errors in the lattice. Because of this coupling, one must reexamine the answers to the questions of stability and beam distribution and see whether the small additional coupling has caused an instability and to what extent it has altered the beam distribution. This is of particular concern when the storage ring is operated near resonances where a small added coupling can cause significant effects. In the case when one is forced to operate near a resonance, it is important to understand the effect of the resonance on stability and beam distribution. Such an operation has become routine, for example, in the B-factory colliders PEP-II and KEK-B when their horizontal betatron tunes are chosen to be close to a half-integer [2,3]. In the case of KEK-B, a special concern to be addressed is due to the planned installation of a crab cavity, which is a natural agent of a linear synchrobetatron resonance.

Regarding the question of linear stability, a general solution was given in the classic paper by Courant and Snyder [4]. They showed that small coupling can cause an instability near a sum, integer, or half-integer resonance but not near a difference resonance. Once this question has been answered, however, one would also like to know the width of the instability. Courant and Snyder answer this question for a particular $x-y$ coupling perturbation. Expressions for the widths of integer and half-integer resonances have also been found for specific perturbations. For the case of synchrobetatron coupling, Hoffstaetter and 
Chao find expressions for the widths of some of the synchrobetatron coupling resonances induced by dispersion at an rf cavity or by a crab cavity [5]. In the present paper, we give general expressions for instability widths, including many of the results of Hoffstaetter and Chao for synchrobetatron coupling, but we also address the question on beam distribution.

A beam of electrons in a storage ring will reach an equilibrium due to a balance between the effects of radiation damping and diffusion [1]. In the absence of nonlinearities, this equilibrium will be a Gaussian function of the phase space coordinates. Subject to further conditions which we will discuss, the Gaussian distribution in phase space can be expressed in the form

$$
f(\vec{z})=\frac{1}{\pi^{3}\left\langle g_{1}\right\rangle\left\langle g_{2}\right\rangle\left\langle g_{3}\right\rangle} \exp \left(-\frac{g_{1}}{\left\langle g_{1}\right\rangle}-\frac{g_{2}}{\left\langle g_{2}\right\rangle}-\frac{g_{3}}{\left\langle g_{3}\right\rangle}\right)
$$

Here, the $g_{1,2,3}$ are quadratic invariants which we will write as $g_{a}=\vec{z}^{T} G_{a} \vec{z}$, with $a=1,2,3, \vec{z}$ being the phase space vector and the $T$ indicating a transpose of the vector. $\left\langle g_{1,2,3}\right\rangle$ are the average values of the invariants over the beam distribution. Under this formulation, the problem reduces to finding the form of the invariants and their average values.

To find the evolution of the $\left\langle g_{a}\right\rangle$, we consider some nonsymplectic process that causes a local change in the beam second moments $\Sigma_{j k}=\left\langle z_{j} z_{k}\right\rangle$. The net change in the $\left\langle g_{a}\right\rangle$ is then given by integrating the corresponding change in the invariant around the ring:

$$
\Delta\left\langle g_{a}\right\rangle=\oint d s \operatorname{Tr}\left[G_{a} \frac{d \Sigma}{d s}\right]
$$

with $\operatorname{Tr}$ representing the trace of a matrix. Thus, in this formulation, the nonsymplectic process acts locally on the moments, causing changes in the invariants which are global quantities, determined from the one-turn map at the given position. In this paper, we consider synchrotron radiation as the nonsymplectic process. In general, however, we could include any nonsymplectic process in this framework. For example, see [6,7] for more information on including intrabeam scattering within a fully coupled formalism.

The $\left\langle g_{a}\right\rangle$ are related to the rms beam emittances by $\left\langle g_{a}\right\rangle=2 \epsilon_{a}$. In case the problem of interest is $2 \mathrm{D}$ (4D phase space), as we will be in the following, the formulation simply changes from $a=1,2,3$ to $a=1,2$.

Formulas for the equilibrium emittances due to synchrotron radiation were given for the uncoupled case in the work by Sands [1] in terms of the so-called synchrotron radiation integrals [8]. In the general case including coupling, an algorithm was given in [9] to numerically compute the equilibrium distribution moments given the linear lattice of the storage ring. This is efficient for precise results, but loses the explicit dependence on the lattice functions that an analytic expression provides. A generalization of the synchrotron radiation integrals was given in $[10,11]$. Reference [11] extends the synchrotron radiation integrals to the case of general coupling, but does not specifically consider the case of a linear resonance. Reference [12] also discusses equilibrium distributions with coupling, including spin polarization, and develops a perturbation theory, but does not cover the case of resonances. In the present work, we consider weak coupling but focus on obtaining analytic expressions near a resonance.

As noted in [11], to compute the emittances exactly using radiation integrals, if one adds a perturbation at one point, one must recalculate all of the lattice functions everywhere. We will formulate our perturbative approach, taking advantage of its approximate nature by treating the coupling as a small perturbation, so that this is not required. Only the uncoupled lattice functions, along with quantities derived from the local perturbation will enter our final expressions.

As also noted in [11], when we are exactly on the resonance, the equilibrium beam distribution is not necessarily well described by a function of the invariants $g_{1,2,3}$ alone, and the basic assumption of our formalism breaks down. This breakdown occurs when particle motion in phase space is dominated not by its symplectic dynamics but by its damping and diffusion effects. In order that the distribution retain the form (1) near resonance, we require the condition

$$
\chi_{x, y, z} \ll|\xi|
$$

where $\chi_{x, y, z}$ are the unperturbed damping decrements (percentage reduction of the $x, y$, and $z$ amplitudes per turn) and $\xi$ is a dimensionless coupling constant to be defined later. ${ }^{1}$ This condition is satisfied over a wide range of practical situations, although it should be checked in specific cases. Reference [11] contains numerical examples relating to this issue. The authors compute the full set of equilibrium moments for a realistic lattice. Expressing these moments in the eigenbasis, one can see to what extent the approximation of Eq. (1) is valid. They do indeed find

\footnotetext{
${ }^{1}$ We argue for Eq. (3) as follows. From [11], we see that being a function of the invariants requires $\chi_{a} \ll|\delta \mu|$ where $\delta \mu$ is the distance from resonance of the eigenphase advances. We will find that $|\delta \mu|=\sqrt{\Delta \mu^{2} \pm \xi^{2}}$ where $\Delta \mu$ is basically the distance from resonance for the uncoupled system. Resonance occurs when $\Delta \mu$ and $\xi$ are of the same order. Near resonance, therefore, $|\delta \mu| \sim \xi$. We will find that the $\chi_{a}$ only change dramatically very close to the resonance, so replacing $\chi_{a}$ by $\chi_{x, y, z}$ should be reasonably good. Thus, if (3) is satisfied, we expect that Eq. (1) is a good approximation except in the extremely close vicinity of an unstable resonance. When the condition (3) fails, one could still use our expressions for coupled eigenvectors to solve for the full set of second moments explicitly.
} 
an example (near a difference resonance) where for very weak coupling, this approximation breaks down. We also note that increased periodicity in a lattice design may further improve the approximation [13].

Linear resonances have been extensively analyzed in accelerator physics. The result of Courant and Snyder previously mentioned can be reframed by saying that instabilities in linear dynamics are possible when two of the eigenvalues of the one-turn map matrix are close to equal. Let us consider the case of 4D transverse-longitudinal phase space. (We refer to this as the case of synchrobetatron coupling. The same framework will apply to the purely transverse $x-y$ coupling as well [15].) The eigenvalues of the uncoupled map are given by $e^{ \pm i \mu_{x}}$ and $e^{ \pm i \mu_{z}}$, where $\mu_{x, z}=2 \pi \nu_{x, z}$ with $\nu_{x, z}$ the horizontal (betatron) and longitudinal (synchrotron) tunes, respectively. The condition of degenerate eigenvalues amounts to either $\nu_{x} \pm$ $\nu_{z}=$ integer, or $\nu_{x}$ or $\nu_{z}$ is an integer or half-integer.

Because the eigenvalues are degenerate at a resonance, we need to consider degenerate perturbation theory. This method is commonly used in quantum mechanics. In that context, one examines how perturbations to the Hamiltonian operator change the energies (eigenvalues) and wave functions (eigenvectors). There is an analogous formulation for classical mechanics which we will develop and apply to our problem. The difference between the two cases is that in the quantum mechanics context, one perturbs a Hermitian matrix. Here we will perturb a symplectic matrix. The framework is quite analogous, but some unique features will emerge in the symplectic case such as the issue of instabilities.

We now give a brief overview of the paper. In Sec. II, we summarize and give definitions regarding the computation of the invariants and their equilibrium values starting from a general one-turn map and the local damping and diffusion from synchrotron radiation. From the one-turn map $M$, we find eigenvalues and eigenvectors. Given an eigenvector $v_{a}$, the invariant matrix is given by

$$
G_{a}=-J\left(v_{a} v_{a}^{\dagger}+v_{a}^{*} v_{a}^{T}\right) J
$$

with $*$ representing complex conjugate, $†$ representing conjugate transpose and $J$ the symplectic inner product matrix given in Eq. (13). We normalize the eigenvectors so that $\boldsymbol{v}^{j} \boldsymbol{v}_{k}=\delta_{j k}$ with $\delta_{j k}$ the Kronecker delta and $\boldsymbol{v}^{j}$ is a "covector" defined by $v^{j}=-i \operatorname{sgn}(j) v_{j}^{\dagger} J$. From the normalized eigenvectors, we also construct a symplectic matrix $U$ using them as columns.

Next we consider a nonsymplectic process which gives rise to constant damping and diffusion matrices. We find that we can describe such a process in terms of a local damping matrix $B$ and a local diffusion matrix $D$. The evolution Eq. (2) simplifies to

$$
\Delta\left\langle g_{a}\right\rangle=-2 \chi_{a}\left\langle g_{a}\right\rangle+\bar{d}_{a}
$$

where the damping decrements $\chi_{a}$ are given by

$$
\chi_{a}=\oint d s\left[A_{a a}+A_{-a-a}\right]
$$

with $A=U^{-1} B U$ and the global diffusion coefficients $\bar{d}_{a}$ are given by

$$
\bar{d}_{a}=\oint d s \operatorname{Tr}\left[G_{a} D\right]
$$

We then give explicit expressions for $B$ and $D$ for synchrotron radiation and show that we reproduce the results of Sands [1] in the uncoupled case.

In Sec. III, we formulate our perturbative approach. We expand the one-turn map and the corresponding eigenvalue equation, writing:

$$
\begin{aligned}
& M=M_{0}+M_{1}+M_{2}+\ldots, \\
& \lambda_{j}=\lambda_{j 0}+\lambda_{j 1}+\lambda_{j 2}+\ldots, \\
& v_{j}=\tilde{v}_{j 0}+v_{j 1}+v_{j 2}+\ldots
\end{aligned}
$$

We consider the case where the one-turn map is near a degeneracy and consider $M_{0}$ to be the map exactly on resonance. $\tilde{v}_{j 0}$ is a linear combination of the $v_{j 0}$, the eigenvectors of the uncoupled map which are degenerate on resonance. The perturbation picks out a specific linear combination of the degenerate eigenvectors. It will be one of the main tasks of this paper to compute $\tilde{\boldsymbol{v}}_{j 0}$. We write $M_{1}=M_{1 \mu}+M_{1 \xi}$ where $M_{1 \mu}$ is the difference from resonance and $M_{1 \xi}$ is any additional perturbation (e.g., coupling). For the case of the integer and half-integer resonance, we find that we need a second order $M_{2 \xi}$ and need to do second order degenerate perturbation theory. For each resonance, we express our results for the eigenphase advance and perturbed eigenvectors in terms of a coupling parameter $\xi$, a splitting parameter $\Delta \mu$, an average shift $\bar{\mu}$, and a phase $\phi$. These are all expressed in terms of the matrix elements $r_{j k}=\boldsymbol{v}^{j 0} P \boldsymbol{v}_{k 0}$ where $M_{1}=P M_{0}$. We find that there are instabilities for all but the difference resonance and the width is given by $\xi>\Delta \mu$.

In Sec. IV, we apply our formalism to two cases of synchrobetatron coupling: dispersion at an rf cavity and the addition of a crab cavity. We give explicit expressions for $\xi, \Delta \mu, \phi$, and $\bar{\mu}$ near each of the resonances, thereby providing analytic formulas for stop-band widths and perturbed eigenvectors.

In Sec. V, we combine the results of Sec. II and III to compute coupled expressions for the invariants $G_{a}$ and also $b_{a}$ and $d_{a}$, the local damping and diffusion coefficients for the eigenmodes. We find that in addition to the coupling mixing together the uncoupled invariants $G_{x}$ and $G_{z}$, there are additional terms $G_{c}$ which oscillate around the ring. These terms could be expressed as linear combinations of 
the extra invariants that exist for the one-turn map exactly on resonance, as discussed further in [10]. By doing our perturbation theory in terms of the eigenvectors, they naturally come out of the analysis. We then find the global damping and diffusion coefficients by integration of these quantities around the ring. The additional mixing terms are found to not contribute much to the global damping and diffusion in typical cases. One notable exception to this is the case of the integer synchrotron tune resonance. Because the synchrotron phase advances very little around the ring, the mixing term does indeed contribute to the global diffusion and one must be careful to include this term. A final notable result of this section is that one of the damping decrements $\chi_{a}$ can become negative near a sum resonance. This indicates an instability which we refer to as an "antidamping instability."

Section VI connects together the results of Secs. IV and $\mathrm{V}$ giving plots and discussion. We use the parameters for the PEP-II LER as an example storage ring to plot the coupled damping and diffusion coefficients and equilibrium emittances as a function of the betatron and synchrotron tunes near each of the resonances. We also examine the question of instability, both due to the Hamiltonian dynamics and due to antidamping.

Section VII contains the conclusion. Further derivations are contained in the appendixes. Appendix A gives derivations related to the invariants and second-moment matrix. In Appendix B we formulate our general perturbation theory. Appendix $\mathrm{C}$ proves a relationship between the coupling parameters for the sum and difference resonances. Appendix D gives further derivations related to the integer and half-integer resonances caused by coupling. Finally, we point out that summaries of many of the results of this paper can be found in Tables I-V, located in Secs. III-V. Once the basic approach is understood, the information contained in these tables would give the main results needed to apply the approach to a new type of perturbation or a new set of parameters.

\section{GENERAL COMPUTATION OF EQUILIBRIUM EMITTANCES}

Consider a general electron storage ring with a given linear lattice. Ignoring the damping and diffusion effects due to synchrotron radiation, we can describe the dynamics at any position $s$ in terms of a one-turn map $M$ :

$$
\vec{z}_{s+C}=M(s) \vec{z}_{s}
$$

where $C$ is the circumference of the ring and where $\vec{z}$ is our phase space coordinate vector (column matrix). The matrix $M$ is symplectic [4] which means

$$
M^{T} J M=J
$$

where a superscript $T$ means taking the transpose of a matrix, and $J$ is the symplectic inner product matrix

$$
J=\left(\begin{array}{cccccc}
0 & 1 & 0 & 0 & 0 & 0 \\
-1 & 0 & 0 & 0 & 0 & 0 \\
0 & 0 & 0 & 1 & 0 & 0 \\
0 & 0 & -1 & 0 & 0 & 0 \\
0 & 0 & 0 & 0 & 0 & 1 \\
0 & 0 & 0 & 0 & -1 & 0
\end{array}\right)
$$

The six eigenvectors and eigenvalues of $M$ satisfy

$$
M v_{k}=\lambda_{k} v_{k}
$$

The index $k$ runs over $\pm 1, \pm 2, \pm 3$. We normalize the eigenvectors such that

$$
v_{j}^{\dagger} J v_{k}=i \operatorname{sgn}(j) \delta_{j k},
$$

where $\operatorname{sgn}(j)$ is 1 for $j>0$ and -1 for $j<0$, and $\dagger$ means taking the complex conjugate and transpose of a matrix (or vector). This normalization condition suggests the definition of an upper indexed object

$$
v^{j} \equiv-i \operatorname{sgn}(j) v_{j}^{\dagger} J
$$

The normalization condition (15) then reads

$$
\boldsymbol{v}^{j} \boldsymbol{v}_{k}=\delta_{j k}
$$

We refer to an eigenvector $v_{k}$ with $k>0$ as a positive mode eigenvector and one with $k<0$ as a negative mode eigenvector. Note that this property of being a positive or negative mode is intrinsic to a given eigenvector and is not a property of the normalization: multiplying by a constant cannot convert a positive mode eigenvector into a negative mode, and vice versa. The positive and negative modes are related as follows:

$$
v_{-k}=i v_{k}^{*}, \quad v^{-k}=-i v^{k *} .
$$

When $M$ describes stable motion in the storage ring, the eigenvalues $\lambda_{k}$ can be expressed as

$$
\lambda_{k}=e^{i \mu_{k}}
$$

with $\mu_{k}$ a real quantity. We refer to $\mu_{k}$ as the eigenphase advance, with $\mu_{k}=2 \pi \nu_{k}$ where $\nu_{k}$ is the eigentune. The positive and negative phase advances are related to each other as

$$
\mu_{-k}=-\mu_{k}
$$

From the $v_{k}$ 's, we construct a matrix using them as columns,

$$
U=\left(\begin{array}{llllll}
v_{1} & v_{-1} & v_{2} & v_{-2} & v_{3} & v_{-3}
\end{array}\right)
$$


Given the normalization, one can show that $U$ is symplectic. We can thus consider $U$ as a (complex) canonical transformation that diagonalizes ${ }^{2} M$. In particular

$$
\begin{aligned}
U^{-1} M U & =e^{\Lambda} \\
& =\left(\begin{array}{cccccc}
e^{i \mu_{1}} & 0 & 0 & 0 & 0 & 0 \\
0 & e^{-i \mu_{1}} & 0 & 0 & 0 & 0 \\
0 & 0 & e^{i \mu_{2}} & 0 & 0 & 0 \\
0 & 0 & 0 & e^{-i \mu_{2}} & 0 & 0 \\
0 & 0 & 0 & 0 & e^{i \mu_{3}} & 0 \\
0 & 0 & 0 & 0 & 0 & e^{-i \mu_{3}}
\end{array}\right) .
\end{aligned}
$$

Let us now discuss the invariants of $M$. Let $g=\vec{z}^{T} G \vec{z}$ be a quadratic invariant of $M$, where $G$ is a symmetric matrix. To be an invariant, $g(s)$ must satisfy the condition $g(s)=$ $g(s+C)$ for all $\vec{z}$, or

$$
M^{T} G M=G .
$$

Note that linear combinations of invariants are still invariants. In the nondegenerate case, there are 3 linearly independent invariants. Given $U$, there is a natural choice for these three invariants. We express them as

$$
g_{a}=\vec{z}^{T} G_{a} \vec{z}, \quad G_{a}=J U H_{a} U^{T} J, \quad a=1,2,3
$$

with

$$
\begin{aligned}
H_{1}=\left(\begin{array}{ccc}
i \sigma_{x} & 0 & 0 \\
0 & 0 & 0 \\
0 & 0 & 0
\end{array}\right), & H_{2}=\left(\begin{array}{ccc}
0 & 0 & 0 \\
0 & i \sigma_{x} & 0 \\
0 & 0 & 0
\end{array}\right), \\
H_{3}=\left(\begin{array}{ccc}
0 & 0 & 0 \\
0 & 0 & 0 \\
0 & 0 & i \sigma_{x}
\end{array}\right), & \sigma_{x}=\left(\begin{array}{ll}
0 & 1 \\
1 & 0
\end{array}\right) .
\end{aligned}
$$

One can verify that these are invariants by using (23), and more details are given in Appendix A. These invariants can also be written directly in terms of the eigenvectors as

$$
G_{a}=-J\left(v_{a} v_{a}^{\dagger}+v_{a}^{*} v_{a}^{T}\right) J .
$$

From this expression, we note two properties of the invariants. First, they are real quantities, and second, they are independent of the overall phase of the eigenvector $v_{a}$. Further, there is no need to allow $a$ to run over negative values. If we do so, Eq. (26) shows that $G_{-a}=G_{a}$. That is, the invariants determined by the negative mode eigenvectors are identical to those from the corresponding positive mode. This is a manifestation of the relationship between $v_{k}$ and $v_{-k}$.

\footnotetext{
${ }^{2}$ Another useful canonical transformation involves taking $\sqrt{2}$ times the real and imaginary parts of the $v_{k}$ 's for the columns of $U$. This is a real canonical transformation and results in a block diagonal $M$ with blocks given by rotation matrices [14]. It is the same as that given in [16]. We discuss this transformation in Appendix A, but otherwise do not make use of it in this work.
}

The invariants $g_{a}$, expressed in terms of normalized eigenvectors in (24) and (26), turn out to be the action variables often used in perturbation theory in classical systems. To see that, let us express the one-turn map as

$$
M=e^{J S} \text {. }
$$

In this form, $S$ has the physical meaning that the effective Hamiltonian describing the one-turn motion around the observation point $s$ is given by $H=\frac{1}{2} \vec{z}^{T} S \vec{z}$. Then using

$$
\begin{aligned}
S & =-J U \Lambda U^{-1}, \\
\Lambda & =\left(\begin{array}{ccc}
i \mu_{1} \sigma_{z} & 0 & 0 \\
0 & i \mu_{2} \sigma_{z} & 0 \\
0 & 0 & i \mu_{3} \sigma_{z}
\end{array}\right), \quad \text { and } \\
\sigma_{z} & =\left(\begin{array}{cc}
1 & 0 \\
0 & -1
\end{array}\right),
\end{aligned}
$$

it follows that

$$
S=\mu_{1} G_{1}+\mu_{2} G_{2}+\mu_{3} G_{3} .
$$

The one-turn effective Hamiltonian is therefore given by

$$
H=\frac{1}{2}\left(\mu_{1} g_{1}+\mu_{2} g_{2}+\mu_{3} g_{3}\right) \text {. }
$$

For the transverse dimension, the $g_{a}$ corresponding to the betatron oscillations reduces to the Courant-Snyder invariant in the uncoupled case. ${ }^{3}$

Now, suppose that the distribution of electrons in the storage ring is given as a function of the invariants $g_{a}$. In particular, let it have a Gaussian distribution given by Eq. (1). In terms of matrices $G_{a}$, the second moments of the distribution

$$
\left\langle z_{i} z_{j}\right\rangle=\Sigma_{i j}
$$

are given by

$$
\Sigma=-\frac{1}{2}\left\langle g_{1}\right\rangle J G_{1} J-\frac{1}{2}\left\langle g_{2}\right\rangle J G_{2} J-\frac{1}{2}\left\langle g_{3}\right\rangle J G_{3} J,
$$

which we derive in Appendix A.

We have now completed the formalism to find the beam equilibrium distribution from the one-turn map $M$. The only missing quantities are $\left\langle g_{1,2,3}\right\rangle$. To find them, we will need to consider the effects of damping and diffusion, which are so far ignored.

\section{A. Damping effect}

In an electron storage ring, the stored electrons give off energy in the form of synchrotron radiation in the bending magnets that is replenished in the rf cavities. This radiation process gives rise to both a damping and a diffusion effect [1]. As mentioned, we consider the one-turn dynamics

\footnotetext{
${ }^{3}$ For the sake of this agreement, we have not included a factor of $1 / 2$ in the definition of the $g_{a}$, Eq. (24).
} 
around a point of observation $s$. We now need to integrate the actions of damping and diffusion for one turn around the same observation point. We will assume that the damping and diffusion effects are very weak, so that they do not affect the particle motion appreciably over one turn of the otherwise symplectic particle motion [see Eq. (3)].

We designate the linear map for $\vec{z}$ from position $s_{1}$ to $s_{2}$, ignoring damping and diffusion, as $T_{s_{1} \rightarrow s_{2}}$. The one-turn map around the observation position $s$ is $M=T_{s \rightarrow s+C}$, with $C$ the ring circumference.

Consider the damping effect first. The action of radiation damping occurs in the bending magnets ${ }^{4}$ and the rf cavities. It is mostly longitudinal synchrotron damping in the bending magnets and transverse betatron damping in the rf cavities. In either case, we define the damping action at position $s^{\prime}$ by a matrix $B\left(s^{\prime}\right)$ for each damping element. In this definition, the transport matrix over a damping element $T_{\text {elem }}$ is modified to become $T_{\text {elem }} \times\left[I-\int d s^{\prime} B\left(s^{\prime}\right)\right]$, where the integration of $s^{\prime}$ is over the damping element under consideration.

Assuming $B\left(s^{\prime}\right)$ is small, we keep only to first order in $B$ when integrating over one turn around position $s$. The result is that the one-turn linear map around $s$ is modified from $M$ to become

$$
M(I-\bar{B})
$$

where

$$
\bar{B}=\int_{s}^{s+C} d s^{\prime} T_{s \rightarrow s^{\prime}}^{-1} B\left(s^{\prime}\right) T_{s \rightarrow s^{\prime}}
$$

Having obtained the symplectic one-turn map $M$ around the observation point $s$ and the integrated damping matrix $\bar{B}$, the one-turn evolution of the beam distribution secondmoment matrix $\Sigma$ is given by

$$
\begin{aligned}
\Sigma(s+C) & =[M(I-\bar{B})] \Sigma(s)[M(I-\bar{B})]^{T} \\
& \approx M \Sigma(s) M^{T}-M \bar{B} \Sigma(s) M^{T}-M \Sigma(s) \bar{B}^{T} M^{T},
\end{aligned}
$$

where we have again kept only first order in the damping matrix, as we do throughout this paper.

\section{B. Diffusion effect}

We next consider the integrated action of diffusion for one turn around position $s$. Let the diffusion action be described by

$$
\frac{d \vec{z}}{d s}=\hat{\xi}
$$

where the vector $\hat{\xi}$ describes the diffusion noise, and has the property that its average over the noise ensemble vanishes, but that

\footnotetext{
${ }^{4}$ In this context, a quadrupole magnet with an off-centered beam is considered a bending magnet.
}

$$
\left\langle\hat{\xi}_{i}\left(s_{1}\right) \hat{\xi}_{j}\left(s_{2}\right)\right\rangle=D_{i j}\left(s_{1}\right) \delta\left(s_{2}-s_{1}\right) .
$$

The brackets \langle\rangle mean averaging over the ensemble of synchrotron radiation events. Quantities $D_{i j}\left(s_{1}\right)$ are the diffusion coefficients. Equation (37) says that the fluctuations from the synchrotron radiation photons form a white noise process which is delta-function correlated in time. Explicit expressions of the matrices $B(s)$ and $D(s)$ are given later in this section.

In response to the diffusion, a particle motion contains an extra term, at the observation point,

$$
\vec{z}(s)=\int_{s}^{s+C} T_{s^{\prime} \rightarrow s+C} \hat{\xi}\left(s^{\prime}\right) d s^{\prime} .
$$

Using this expression, one computes $z_{i} z_{j}$ at position $s$. Averaging $z_{i} z_{j}$ over the beam distribution and over the ensemble of possible $\hat{\xi}$ 's, we obtain an extra term contributing to the second-moment matrix $\Sigma$ for its one-turn evolution,

$$
\bar{D}(s)=\int_{s}^{s+C} d s^{\prime} T_{s^{\prime} \rightarrow s+C} D\left(s^{\prime}\right) T_{s^{\prime} \rightarrow s+C}^{T} .
$$

Physically, $\int D\left(s^{\prime}\right) d s^{\prime}$ over a storage ring element gives the contribution of diffusion to the $\Sigma$ second moments due to this element. This contribution is then transferred to position $s$ by the map $T_{s^{\prime} \rightarrow s+C}$ to give the integrated $\bar{D}$.

\section{Evolution of beam distribution}

Combining the results of (35) and (39) then gives the net one-turn evolution ${ }^{5}$

$$
\begin{aligned}
\Sigma(s+C)= & M \Sigma(s) M^{T}-M \bar{B} \Sigma(s) M^{T}-M \Sigma(s) \bar{B}^{T} M^{T} \\
& +\bar{D}(s)
\end{aligned}
$$

To find the exact equilibrium distribution, we stipulate in Eq. (40) that $\Sigma(s+C)=\Sigma(s)=\Sigma_{\mathrm{eq}}(s)$. This equation can be solved for $\Sigma_{\text {eq }}$ directly using eigenanalysis $[11,17]$. For our purposes here, however, we assume that the distribution stays a function of the invariants. The average value of the invariant is given by

$$
\left\langle g_{a}\right\rangle=\operatorname{Tr}\left(G_{a} \Sigma\right), \quad a=1,2,3 .
$$

Using (23), (40), and (41), along with the cyclic property of the trace, we obtain the evolution equation for $\left\langle g_{a}\right\rangle$,

$$
\left\langle g_{a}\right\rangle(s+C)-\left\langle g_{a}\right\rangle(s)=-2 \operatorname{Tr}\left(\bar{B} \Sigma G_{a}\right)+\operatorname{Tr}\left(G_{a} \bar{D}\right) .
$$

We then use (24) and (32) and $U^{T} G_{a} U=J H_{a} J$ (using the symplectic property of $U$ ) to obtain, after some algebra,

\footnotetext{
${ }^{5}$ Note that we have assumed that both the damping and diffusion terms are small in comparison to the symplectic evolution, so that we could compute the damping effect while ignoring the diffusion and vice versa.
} 


$$
\left\langle g_{a}\right\rangle(s+C)-\left\langle g_{a}\right\rangle(s)=-2 \chi_{a}\left\langle g_{a}\right\rangle+\bar{d}_{a},
$$

where the global eigenmode damping decrements per turn $\chi_{a}$ are given by

$$
\chi_{a}=\bar{A}_{a a}+\bar{A}_{-a-a}, \quad a=1,2,3,
$$

where

$$
\bar{A}=U^{-1} \bar{B} U,
$$

and the one-turn eigenmode diffusion coefficients $\bar{d}_{a}$ are

$$
\bar{d}_{a}=\operatorname{Tr}\left(G_{a} \bar{D}\right), \quad a=1,2,3 .
$$

From (43), we see that the equilibrium value of the invariants are given by

$$
\left\langle g_{a}\right\rangle_{\mathrm{eq}}=\frac{\bar{d}_{a}}{2 \chi_{a}}, \quad a=1,2,3 .
$$

It may be useful to express the global damping decrements $\chi_{a}$ and the global diffusion coefficients $\bar{d}_{a}$ in an integral form. Writing out (45) explicitly gives

$$
\begin{aligned}
\bar{A} & =U^{-1}(s) \bar{B}(s) U(s)=\int_{s}^{s+C} U^{-1}\left(s^{\prime}\right) B\left(s^{\prime}\right) U\left(s^{\prime}\right) d s^{\prime} \\
& \equiv \oint A\left(s^{\prime}\right) d s^{\prime},
\end{aligned}
$$

where we have used the fact that the $U$ matrix of eigenvectors transforms as $U(s)=T_{s \rightarrow s^{\prime}} U\left(s^{\prime}\right)$, and defined the local $A$ matrix in terms of the local damping matrix as ${ }^{6}$

$$
A\left(s^{\prime}\right)=U^{-1}\left(s^{\prime}\right) B\left(s^{\prime}\right) U\left(s^{\prime}\right) .
$$

The matrix $\bar{A}$ is independent of the observation position $s$, although $\bar{B}$ in general depends on $s$. We can thus write the global damping decrements as

$$
\chi_{a}=\oint\left[A_{a a}\left(s^{\prime}\right)+A_{-a-a}\left(s^{\prime}\right)\right] d s^{\prime} .
$$

We refer to the integrand as the local damping coefficient and write

$$
b_{a}=A_{a a}+A_{-a-a}
$$

so that

$$
\chi_{a}=\oint b_{a}\left(s^{\prime}\right) d s^{\prime}
$$

For the diffusion coefficients, we use (39) and (46), and the transformation property of the invariant $G_{a}\left(s^{\prime}\right)=$ $T_{s^{\prime} \rightarrow s+C}^{T} G_{a}(s) T_{s^{\prime} \rightarrow s+C}$ to derive

$$
\bar{d}_{a}=\oint \operatorname{Tr}\left[G_{a}\left(s^{\prime}\right) D\left(s^{\prime}\right)\right] d s^{\prime} \equiv \oint d_{a}\left(s^{\prime}\right) d s^{\prime},
$$

\footnotetext{
${ }^{6}$ The matrix $A$ depends on the overall phases of the eigenvectors $v_{k}$ in $U$. However, the diagonal elements are independent of these phases, and only the diagonal elements enter the evolution equations for the invariants.
}

where we have defined the local diffusion coefficient

$$
d_{a}=\operatorname{Tr}\left[G_{a} D\right] .
$$

The global diffusion coefficients $\bar{d}_{a}$ are independent of $s$. It follows that the equilibrium emittances $\frac{1}{2}\left\langle g_{a}\right\rangle_{\mathrm{eq}}$ are also global quantities, independent of $s$.

Thus, to find the equilibrium emittance, we must find the local invariants $G_{a}$ and the $U$ matrix constructed from the eigenvectors of $M$ using (21) and (24). Given these and local damping and diffusion matrices $B$ and $D$, we find the local damping and diffusion coefficients $b_{a}$ and $d_{a}$ from (49), (51), and (54). Finally, we integrate these around the ring to find the global damping decrements and global diffusion coefficients which give us the equilibrium emittances.

\section{Sum rules}

The sum of the local damping coefficients gives the trace of $A(s)$. From this we derive

$$
b_{1}+b_{2}+b_{3}=\operatorname{Tr}\left(U^{-1} B U\right)=\operatorname{Tr}(B)=b_{x}+b_{y}+b_{z},
$$

where $b_{x}, b_{y}$, and $b_{z}$ are the damping coefficients in uncoupled coordinates. Thus, regardless of the details of the Hamiltonian dynamics [contained in $U(s)$ ], the sum of the $b_{a}$ is unchanged. This is a local sum rule for the damping coefficients. Integrating this equation around the ring, we get a global sum rule,

$$
\chi_{1}+\chi_{2}+\chi_{3}=\chi_{x}+\chi_{y}+\chi_{z}
$$

where $\chi_{x, y, z}$ are the global damping decrements for the uncoupled case. Equation (56) is a manifestation of the well-known Robinson theorem [18].

Under some specific conditions, one can also obtain a sum rule for the invariants and diffusion coefficients. In particular, consider a 2D coupled storage ring. Equation (29) says that

$$
\mu_{1} G_{1}+\mu_{2} G_{2}=S .
$$

In later sections, we will formulate a perturbation theory in which we start with an uncoupled, degenerate map and consider the effects of small coupling and small deviation from degeneracy. Let us write $\mu_{x 0}$ and $\mu_{z 0}$ for the phase advances at degeneracy (e.g. $\mu_{x 0}=-\mu_{z 0}$ for sum resonances, and $\mu_{x 0}=\mu_{z 0}$ for difference resonances) and $S_{0}$ for the effective Hamiltonian matrix at the degeneracy. At the degeneracy, then, we have

$$
\mu_{x 0} G_{x}+\mu_{z 0} G_{z}=S_{0}
$$

Breaking the degeneracy and adding a small coupling amount to adding small perturbations to the one-turn map. This will add small corrections to $S_{0}$, and $\mu_{x 0, z 0}$ to first order in the perturbations. The invariants $G_{1,2}$, however can have large changes depending on the perturbation, 
as will be clear in later sections. Then, taking (57) to lowest order and setting it equal to (58), we get

$$
\mu_{x 0} G_{1}+\mu_{z 0} G_{2}=\mu_{x 0} G_{x}+\mu_{z 0} G_{z} .
$$

This is a sum rule for the invariants. For the case of the sum/difference resonance it says

$$
\begin{array}{ll}
G_{1}-G_{2}=G_{x}-G_{z} & \text { sum res, } \\
G_{1}+G_{2}=G_{x}+G_{z} & \text { dif res. }
\end{array}
$$

For the integer resonance in the longitudinal motion, we have $\mu_{z 0}=0$, in which case this sum rule tells us that to lowest order, the invariant in the $x$ dimension is unchanged, ${ }^{7}$

$$
G_{1}=G_{x} \quad \text { int } z \text { res. }
$$

Similarly,

$$
G_{2}=G_{z} \quad \text { int } x \text { res. }
$$

Now, the local diffusion coefficients $d_{a}$ are given by $d_{a}=\operatorname{Tr}\left(G_{a} D\right)$, where $D$ is the diffusion matrix. Multiplying the above sum rules on the right by the matrix $D$ and then taking a trace gives sum rules for the local diffusion coefficients. The results for the sum/difference and integer resonances are

$$
\begin{array}{rlrl}
d_{1}-d_{2} & =d_{x}-d_{z}=\text { invariant } & & \text { sum res, } \\
d_{1}+d_{2} & =d_{x}+d_{z}=\text { invariant } & & \text { dif res, } \\
d_{1} & =d_{x} & \text { int } z \text { res, } & \\
d_{2} & =d_{z} \quad \text { int } x \text { res. } &
\end{array}
$$

We can also integrate this equation around the ring to get sum rules for the global diffusion coefficients,

$$
\begin{array}{rlrl}
\bar{d}_{1}-\bar{d}_{2} & =\bar{d}_{x}-\bar{d}_{z}=\text { invariant } & & \text { sum res, } \\
\bar{d}_{1}+\bar{d}_{2} & =\bar{d}_{x}+\bar{d}_{z}=\text { invariant } & & \text { dif res, } \\
\bar{d}_{1} & =\bar{d}_{x} \quad & \text { int } z \text { res }, & \\
\bar{d}_{2} & =\bar{d}_{z} \quad \text { int } x \text { res, } &
\end{array}
$$

where the $\bar{d}_{a}$ are the integrated $d_{a}$ as given in (53).

From the sum rule (64) for sum and difference resonances (global version), and using (47), we have

$$
\begin{array}{ll}
\chi_{1}\left\langle g_{1}\right\rangle_{\mathrm{eq}}-\chi_{2}\left\langle g_{2}\right\rangle_{\mathrm{eq}}=\text { invariant } & \text { sum res, } \\
\chi_{1}\left\langle g_{1}\right\rangle_{\mathrm{eq}}+\chi_{2}\left\langle g_{2}\right\rangle_{\mathrm{eq}}=\text { invariant } & \text { dif res. }
\end{array}
$$

Since $\chi_{1,2}$ must be positive for stable motion, it follows

\footnotetext{
${ }^{7}$ One might be tempted to write the sum rule for the invariants as $\mu_{1} G_{1}+\mu_{2} G_{2}=\mu_{x} G_{x}+\mu_{z} G_{z}$ and note that near an integer $(x)$ resonance $\mu_{2} \approx \mu_{z}$ and $G_{2} \approx G_{z}$ and hence conclude that $G_{1}=\left(\mu_{x} / \mu_{1}\right) G_{x}$. This reasoning is incorrect since the sum rule is only valid to lowest order, and to lowest order $\mu_{1}=\mu_{x 0}=0$. Equation (257) later shows that this flawed reasoning misses a term.
}

that this sum rule imposes a stability condition for particle motion. For example, in case of coupling between the two transverse betatron motions, Eq. (65) implies that the motion is stable near a difference resonance and possibly unstable near a sum resonance. This is a familiar result involving the sum rule of equilibrium beam emittances [4]. The present formalism therefore contains in one framework the Robinson sum rule and the emittance sum rule near linear resonances.

In the case where the coupling near a sum/difference resonance occurs in synchrobetatron space, and the operation is above transition, we find that $\mu_{z}=-\mu_{s}$ (see later) where $\mu_{s}$ is the usual (positive) synchrotron phase advance per turn. Thus, in terms of $\mu_{s}$, there is a sign reversal in the definition of degeneracy so that a sum resonance has $\mu_{x 0}=\mu_{s}$ and difference resonance has $\mu_{x 0}=-\mu_{s}$. In terms of $\mu_{s}$, then, stability applies near a sum resonance and instability occurs near a difference resonance. This is also a familiar result [19], associated with the longitudinal negative mass above transition. In the present paper, however, we make the choice to relate our definitions of resonance to $\nu_{z}$ so that as in the case of $x-y$ coupling, the difference resonance is stable and the sum resonance is unstable. This has the advantage of permitting a uniform treatment of synchrobetatron coupling and transverse betatron coupling. To reiterate, by "sum resonance," we mean $\nu_{x}+\nu_{z}$ is near an integer, and for a "difference resonance," $\nu_{x}-\nu_{z}$ is near an integer.

\section{E. Expressions for $B(s)$ and $D(s)$}

For completeness, we give explicit expressions of $B(s)$ and $D(s)$ here for the synchrobetatron coupled case. Our coordinates are $\left(x, x^{\prime}, z, \delta\right)$ where $x$ and $z$ are the particle's horizontal and longitudinal displacements relative to the beam center, $x^{\prime}=p_{x} / p_{z}$ is the slope of the particle's motion in $x$, and $\delta=\left(p_{z}-P_{0}\right) / P_{0}$ where $P_{0}$ is the design reference momentum.

Ignoring the opening angle effect, the diffusion due to synchrotron radiation only happens in the coordinate $\delta$, and

$$
D(s)=\left(\begin{array}{cccc}
0 & 0 & 0 & 0 \\
0 & 0 & 0 & 0 \\
0 & 0 & 0 & 0 \\
0 & 0 & 0 & d(s)
\end{array}\right),
$$

where [1]

$$
d(s)=\frac{55}{48 \sqrt{3}} \alpha_{0} \frac{\gamma^{5}}{|\rho(s)|^{3}}\left(\frac{\hbar}{m c}\right)^{2},
$$

with $\alpha_{0}$ the fine structure constant, $\gamma$ the relativistic energy factor, $\rho(s)$ the bending radius of the bending magnets, $\hbar$ the reduced Planck constant, and $m$ the electron rest mass. As we see from the $\rho(s)$ in the denominator, diffusion only happens in the bending magnets. 
Later in our applications, it will be convenient to make a transformation from $\vec{z}$ to the betatron coordinates $\vec{z}_{\beta}=\mathcal{B} \vec{z}$ by a coordinate transformation

$$
\mathcal{B}=\left(\begin{array}{cccc}
1 & 0 & 0 & -\eta \\
0 & 1 & 0 & -\eta^{\prime} \\
\eta^{\prime} & -\eta & 1 & 0 \\
0 & 0 & 0 & 1
\end{array}\right)
$$

where $\eta$ and $\eta^{\prime}$ are the dispersion function and its slope at position $s$. It follows that we will need to define

$$
D_{\beta}=\mathcal{B} D \mathcal{B}^{T}=d\left(\begin{array}{cccc}
\eta^{2} & \eta \eta^{\prime} & 0 & -\eta \\
\eta \eta^{\prime} & \eta^{\prime 2} & 0 & -\eta^{\prime} \\
0 & 0 & 0 & 0 \\
-\eta & -\eta^{\prime} & 0 & 1
\end{array}\right),
$$

which we write in terms of $2 \times 2$ submatrices as

$$
D_{\beta}=\left(\begin{array}{ll}
D_{x x} & D_{x z} \\
D_{z x} & D_{z z}
\end{array}\right)
$$

Damping occurs in the bending magnets and in the rf cavity. At an rf cavity, the particle gains back the average energy by the amount $U_{0}$, which it lost due to radiation one turn around the ring. It follows that the rf cavity contributes to a damping [1]

$$
x^{\prime} \rightarrow x^{\prime}\left(1-\frac{U_{0}}{c P_{0}}\right)
$$

which could be described as

$$
B_{\text {cav }}(s)=\left(\begin{array}{cccc}
0 & 0 & 0 & 0 \\
0 & \frac{U_{0}}{c P_{0}} \delta\left(s-s_{c}\right) & 0 & 0 \\
0 & 0 & 0 & 0 \\
0 & 0 & 0 & 0
\end{array}\right),
$$

where $s_{c}$ is the location of the cavity. In case of multiple rf cavities at positions $s_{c i}$, we define

$$
b_{x}(s)=\sum_{i} \frac{U_{0 i}}{c P_{0}} \delta\left(s-s_{c i}\right),
$$

with $\sum_{i} U_{0 i}=U_{0}$, i.e., the net energy loss is $U_{0}$.

For the damping effect due to the bending magnets, we have

$$
B_{\text {bend }}(s)=\left(\begin{array}{cccc}
0 & 0 & 0 & 0 \\
0 & 0 & 0 & 0 \\
0 & 0 & 0 & 0 \\
b_{\delta x} & 0 & 0 & b_{z}
\end{array}\right) .
$$

The $b_{z}$ term comes from the fact that a higher energy electron radiates more whereas the $b_{\delta x}$ term has two parts. The first comes from the fact that a particle with a larger value of $x$ takes a longer path through the magnet and the second from the fact that the power radiated depends on the magnetic field, which in turn depends on $x$ if the vertical magnetic field strength $B_{y}$ contains a quadrupole component. In separated function dipoles, this second part van- ishes. The first is proportional to $\frac{1}{\rho}$. Specifically, we have

$$
b_{z}=P_{\gamma} c P_{0}, \quad b_{\delta x}=\frac{P_{\gamma}}{2 c E_{0}}\left(\frac{1}{\rho}+\frac{2}{B_{y}} \frac{\partial B_{y}}{\partial x}\right),
$$

where $c$ is the speed of light, $E_{0}=c P_{0}$ at high energy, is the reference energy of the particle, $P_{\gamma}$ is the instantaneous radiated power given by

$$
P_{\gamma}=\frac{e^{2} c^{3} C_{\gamma}}{2 \pi} E_{0}^{2} B_{y}^{2}
$$

$e$ is the electron charge, $r_{e}$ is the electron classical radius, and the constant

$$
C_{\gamma}=\frac{4 \pi}{3} \frac{r_{e}}{\left(m c^{2}\right)^{3}}=8.85 \times 10^{-5} \text { meter }-\mathrm{GeV}^{-3} .
$$

$P_{\gamma}$ is only nonzero in the bending magnets. This is why we have written $B_{\text {bend }}$ in Eq. (74).

Altogether, then, we have

$$
B(s)=\left(\begin{array}{cccc}
0 & 0 & 0 & 0 \\
0 & b_{x}(s) & 0 & 0 \\
0 & 0 & 0 & 0 \\
b_{\delta x}(s) & 0 & 0 & b_{z}(s)
\end{array}\right) .
$$

For later applications, we will again need to transform the damping matrix to the betatron coordinates. We then calculate

$$
\begin{aligned}
B_{\beta}(s) & =\mathcal{B B} \mathcal{B}^{-1} \\
& =\left(\begin{array}{cccc}
-\eta b_{\delta x} & 0 & 0 & -\eta\left(b_{z}+\eta b_{\delta x}\right) \\
-\eta^{\prime} b_{\delta x} & b_{x} & 0 & \eta^{\prime}\left(b_{x}-b_{z}-\eta b_{\delta x}\right) \\
0 & -b_{x} \eta & 0 & -b_{x} \eta \eta^{\prime} \\
b_{\delta x} & 0 & 0 & b_{z}+\eta b_{\delta x}
\end{array}\right) .
\end{aligned}
$$

Let us write this in terms of $2 \times 2$ submatrices as

$$
B_{\beta}=\left(\begin{array}{cc}
B_{x x} & B_{x z} \\
B_{z x} & B_{z z}
\end{array}\right)
$$

\section{F. Uncoupled ring}

A typical storage ring is designed to be uncoupled. If we use betatron coordinates, defined by $\vec{z}_{\beta}=\mathcal{B} \vec{z}$ with $\mathcal{B}$ defined in (68), then the linear one-turn map for the ring at some position $s$ is of the form

$$
M_{\text {uncoupled }}=\left(\begin{array}{cc}
M_{x} & 0 \\
0 & M_{z}
\end{array}\right) \text {. }
$$

Because $M_{x}$ and $M_{z}$ are symplectic, following Courant and Snyder, we can write them in the form

$$
\begin{aligned}
M_{x} & =\cos \mu_{x} I+\sin \mu_{x} J_{x}=e^{\mu_{x} J_{x},} \\
J_{x} & =\left(\begin{array}{cc}
\alpha_{x} & \beta_{x} \\
-\gamma_{x} & -\alpha_{x}
\end{array}\right),
\end{aligned}
$$


and

$$
\begin{aligned}
M_{z} & =\cos \mu_{z} I+\sin \mu_{z} J_{z}=e^{\mu_{z} J_{z}}, \\
J_{z} & =\left(\begin{array}{cc}
\alpha_{z} & \beta_{z} \\
-\gamma_{z} & -\alpha_{z}
\end{array}\right) .
\end{aligned}
$$

Here, $\beta_{x}, \alpha_{x}=-2 \beta_{x}^{\prime}$, and $\gamma_{x}=\left(1+\alpha_{x}^{2}\right) / \beta_{x}$ are the usual Courant-Snyder lattice parameters. They are periodic with period $C$; e.g. $\beta_{x}(s+C)=\beta_{x}(s)$. Note that adding integer multiples of $2 \pi$ to $\mu_{x}$ and $\mu_{z}$ does not change the one-turn map. We will thus, except where otherwise noted, assume that an appropriate multiple has been added (subtracted) so that

$$
\mu_{x, z} \in[-\pi, \pi]
$$

To find the corresponding lattice parameters for $z$, let us consider a model storage ring with one rf cavity. The transfer matrix for the rf cavity can then be written as

$$
T_{\text {cav }}=\left(\begin{array}{cc}
1 & 0 \\
\frac{r}{a} & 1
\end{array}\right)
$$

where $r=4 \sin ^{2} \frac{\mu_{z}}{2}$, with $\mu_{z}$ the synchrotron phase advance. ${ }^{8}$ The transfer matrix from the rf cavity to another position $s$ is

$$
T_{z \rho}(s)=\left(\begin{array}{cc}
1 & -a \check{\alpha} \\
0 & 1
\end{array}\right) .
$$

The subscript tells us that this is the longitudinal transfer matrix taking bends into account. Here, $a=C \alpha_{c}$ with $\alpha_{c}$ the momentum compaction factor, ${ }^{9}$ and

$$
\check{\alpha}=\frac{1}{a} \int_{s_{c}}^{s} \frac{\eta\left(s^{\prime}\right)}{\rho\left(s^{\prime}\right)} d s^{\prime},
$$

with $s_{c}$ the position of the rf cavity, $\eta$ the dispersion and $\rho$ the dipole bending radius. We note that $\check{\alpha}$ depends on the two positions $s_{c}$ and $s$ and ranges from 0 (when $s=s_{c}$ ) to 1 (when $s=s_{c}+C$ ). We call $\alpha_{c} \breve{\alpha}$ the partial momentum compaction. The one-turn map at an arbitrary position $s$ is then

$$
M_{z}=\left(\begin{array}{cc}
1-r \check{\alpha} & -a[1+r \check{\alpha}(\check{\alpha}-1)] \\
\frac{r}{a} & 1-r+r \check{\alpha}
\end{array}\right) .
$$

One can verify that the eigenvalues of $M_{z}$ are indeed $e^{ \pm i \mu_{z}}$. Comparing the two different forms for $M_{z}$, we

\footnotetext{
${ }^{8}$ For multiple cavities, we consider them to all be lumped into a single cavity with the correct strength to give the appropriate $\mu_{z}$

${ }^{9}$ We have assumed that the beam is well above transition. For the general case, we replace, $\alpha_{c}$ with the slip factor, $\alpha_{c}-1 / \gamma^{2}$ where $\gamma$ is the relativistic factor.
}

identify

$$
\begin{aligned}
& \beta_{z}=-\frac{a[1+\check{\alpha} r(\check{\alpha}-1)]}{\sin \mu_{z}}, \quad \gamma_{z}=-\frac{r}{a \sin \mu_{z}}, \\
& \alpha_{z}=(1-2 \check{\alpha}) \tan \frac{\mu_{z}}{2} .
\end{aligned}
$$

The synchrotron phase advance per turn $\mu_{z}$ is typically small. Taking lowest order in $\mu_{z}$ we get

$$
\beta_{z}=-\frac{a}{\mu_{z}}, \quad \gamma_{z}=-\frac{\mu_{z}}{a}, \quad \alpha_{z}=\frac{\mu_{z}}{2}(1-2 \check{\alpha}) .
$$

Note that in order to preserve the symplectic requirement that $\beta_{z} \gamma_{z}=1+\alpha_{z}^{2}$, we have to keep higher order in $\mu_{z}$ in $\beta_{z}$ and $\gamma_{z}$.

We require that $\beta_{z}$ and $\gamma_{z}$ be positive. This means that $\mu_{z}$ must be negative (assuming $a>0$, which is true above transition). Thus, designating the usual (positive) synchrotron phase as $\mu_{s}$, we have $\mu_{z}=-\mu_{s}$. In terms of $\mu_{s}$ then,

$$
\beta_{z}=\frac{a}{\mu_{s}}, \quad \gamma_{z}=\frac{\mu_{s}}{a}, \quad \alpha_{z}=\frac{-\mu_{s}}{2}(1-2 \check{\alpha}) .
$$

Given the general forms (82) and (83) $M_{x}$ and $M_{z}$, we can express the eigenvectors of $M_{\text {uncoupled }}$ as

$$
v_{x}=\frac{1}{\sqrt{2}}\left(\begin{array}{c}
\sqrt{\beta_{x}} \\
\frac{i-\alpha_{x}}{\sqrt{\beta_{x}}} \\
0 \\
0
\end{array}\right), \quad v_{z}=\frac{1}{\sqrt{2}}\left(\begin{array}{c}
0 \\
0 \\
\sqrt{\beta_{z}} \\
\frac{i-\alpha_{z}}{\sqrt{\beta_{z}}}
\end{array}\right),
$$

with corresponding eigenvalues $e^{i \mu_{x}}$ and $e^{i \mu_{z}}$, which can be checked by direct multiplication. The above $v_{x}$ and $v_{z}$ are positive modes, and the corresponding negative modes are $v_{-x}=i v_{x}^{*}$ and $v_{-z}=-i v_{z}^{*}$. Using the notation in (16), we can express the normalization as $\boldsymbol{v}^{x} \boldsymbol{v}_{x}=\boldsymbol{v}^{z} \boldsymbol{v}_{z}=$ $\boldsymbol{v}^{-x} \boldsymbol{v}_{-x}=\boldsymbol{v}^{-z} \boldsymbol{v}_{-z}=1$ and all other combinations give 0 . To be explicit, because $v_{x}$ is a positive mode (likewise $\left.v_{z}\right), v^{x}=-i v_{x}^{\dagger} J$

\section{G. Transformation of eigenvectors around ring}

Here, we consider how the eigenvectors transform around the ring. This is necessary because we will want to know how the damping and diffusion coefficients transform around the ring, and they depend on the local eigenvectors. The eigenvectors transform in the same way as the phase space coordinate $\vec{z}$ does, i.e., with the transfer matrix from position $s_{1}$ to position $s_{2}$. We express the transfer matrix in terms of the Courant-Snyder lattice functions and the phase advance from $s_{1}$ to $s_{2}, \psi_{x 12}$, which is related to $\beta_{x}(s)$ by

$$
\psi_{x 12}=\int_{s_{1}}^{s_{2}} \frac{d s}{\beta_{x}(s)} .
$$

In betatron coordinates, the transfer matrix is given by 


$$
T_{0 \beta}\left(s_{1} \rightarrow s_{2}\right) \equiv T_{12 \beta}=\left(\begin{array}{cccc}
R_{11} & R_{12} & 0 & 0 \\
R_{21} & R_{22} & 0 & 0 \\
0 & 0 & 1 & -a \check{\alpha} \\
0 & 0 & 0 & 1
\end{array}\right),
$$

where $a \check{\alpha}$ is $C$ times the partial momentum compaction factor from $s_{1}$ to $s_{2}$, and

$$
\begin{aligned}
R_{11} & =\sqrt{\frac{\beta_{2}}{\beta_{1}}}\left(\cos \psi_{x 12}+\alpha_{1} \sin \psi_{x 12}\right), \\
R_{12} & =\sqrt{\beta_{1} \beta_{2}} \sin \psi_{x 12}, \\
R_{21} & =\frac{1}{\sqrt{\beta_{1} \beta_{2}}}\left[\left(\alpha_{1}-\alpha_{2}\right) \cos \psi_{x 12}-\left(1+\alpha_{1} \alpha_{2}\right) \sin \psi_{x 12}\right] \\
R_{22} & =\sqrt{\frac{\beta_{1}}{\beta_{2}}}\left(\cos \psi_{x}-\alpha_{2} \sin \psi_{x 12}\right),
\end{aligned}
$$

where $\beta_{1}=\beta_{x}\left(s_{1}\right)$, etc., When $s_{2}=s_{1}+C, \psi_{x 12}=\mu_{x}$.

We have not written the longitudinal transfer matrix in terms of $\beta_{z}, \alpha_{z}$, and $\gamma_{z}$ for reasons of convenience. We have assumed that we have not crossed an rf cavity from $s_{1}$ to $s_{2}$. In the case that we do, we need to also multiply by (85). To lowest order in $\mu_{s}$,

$$
\psi_{z 12}=\check{\alpha} \mu_{s},
$$

where $\check{\alpha}$ is integrated from $s_{1}$ to $s_{2}$. This can be derived by setting $R_{12}$ for the $z$ transformation $(-a \check{\alpha}$ ) equal to $\sqrt{\beta_{z 1} \beta_{z 2}} \sin \psi_{z 12}$. In the general case with multiple rf cavities, we would need to compute the resulting $\beta_{z}, \alpha_{z}$ and $\gamma_{z}$, but otherwise the formalism remains the same. Note, however, that we assume that the dispersion at each of the rf cavities is 0 in the computation of the $\alpha_{z}, \beta_{z}$, and $\gamma_{z}$. These are uncoupled lattice functions. We treat dispersion at an rf cavity as a perturbation and later give an example for the case of a single cavity.

The transfer map in betatron coordinates is related to the transfer map in real coordinates by

$$
T_{12 \beta}=\mathcal{B}_{2} T_{12} \mathcal{B}_{1}^{-1},
$$

where $\mathcal{B}_{1}$ and $\mathcal{B}_{2}$ are the betatron coordinate transformation matrices given in (68) involving the dispersion at $s_{1}$ and $s_{2}$ respectively.

Applying $T_{12 \beta}$ to the eigenvectors, we can show

$$
T_{12 \beta} \boldsymbol{v}_{x}\left(s_{1}\right)=e^{i \psi_{x 12}} \boldsymbol{v}_{x}\left(s_{2}\right), T_{12 \beta} \boldsymbol{v}_{z}\left(s_{1}\right)=e^{i \psi_{z 12}} \boldsymbol{v}_{z}\left(s_{2}\right),
$$

where $v_{x}\left(s_{2}\right)$ and $v_{z}\left(s_{2}\right)$ are expressions (92) with the lattice functions advanced to position $s_{2}$.

\section{H. Uncoupled Invariants and Diffusion Coefficients}

Using the uncoupled eigenvectors $v_{x, z}$, Eq. (92), together with Eq. (26) we find the uncoupled invariants

$$
G_{x}=\left(\begin{array}{cccc}
\gamma_{x} & \alpha_{x} & 0 & 0 \\
\alpha_{x} & \beta_{x} & 0 & 0 \\
0 & 0 & 0 & 0 \\
0 & 0 & 0 & 0
\end{array}\right)
$$

$$
G_{z}=\left(\begin{array}{cccc}
0 & 0 & 0 & 0 \\
0 & 0 & 0 & 0 \\
0 & 0 & \gamma_{z} & \alpha_{z} \\
0 & 0 & \alpha_{z} & \beta_{z}
\end{array}\right)
$$

For the $x$ direction, we find the standard Courant-Snyder invariant,

$$
g_{x}=\gamma_{x} x_{\beta}^{2}+2 \alpha_{x} x_{\beta} x_{\beta}^{\prime}+\beta_{x} x_{\beta}^{\prime 2},
$$

where we recall that we are using the $\vec{z}_{\beta}$ betatron coordinates defined in Eq. (68).

The uncoupled local diffusion coefficients are given by

$$
\begin{gathered}
d_{x}=\operatorname{Tr}\left(G_{x} D_{\beta}\right)=d \mathcal{H}_{x}, \\
d_{z}=\operatorname{Tr}\left(G_{z} D_{\beta}\right)=d \beta_{z} \approx d \frac{a}{\mu_{s}} .
\end{gathered}
$$

We use the standard definition of $\mathcal{H}_{x}$

$$
\mathcal{H}_{x}=\gamma_{x} \eta_{x}^{2}+2 \alpha_{x} \eta_{x} \eta_{x}^{\prime}+\beta_{x} \eta^{\prime 2}
$$

The global diffusion coefficients $\bar{d}_{x}$ and $\bar{d}_{z}$ are given by integrating these quantities around the ring.

\section{Uncoupled global damping decrements}

To find the damping decrements, we use Eqs. (48) and (50). Using betatron coordinates, in the uncoupled case, the $U$ matrix and its inverse are block diagonal,

$$
U=\left(\begin{array}{cc}
U_{x} & 0 \\
0 & U_{z}
\end{array}\right), \quad U^{-1}=\left(\begin{array}{cc}
U_{x}^{-1} & 0 \\
0 & U_{z}^{-1}
\end{array}\right)
$$

where $U_{x, z}$ and $U_{x, z}^{-1}$ are $2 \times 2$ matrices. All these quantities depend on the position in the ring $s$. Equation (50) then says

$$
\chi_{x}=\oint \operatorname{Tr}\left[U_{x}^{-1}(s) B(s) U_{x}(s)\right] d s=\oint \operatorname{Tr}\left[B_{x x}(s)\right] d s,
$$

and likewise for $z$,

$$
\chi_{z}=\oint \operatorname{Tr}\left[B_{z z}\right] d s
$$


Thus, in the uncoupled case, we find that

$$
\begin{aligned}
& \chi_{x}=\oint\left[b_{x}(s)-\eta b_{\delta x}(s)\right] d s \equiv \frac{U_{0}}{2 E_{0}}(1-\mathcal{D}) \equiv \frac{U_{0}}{2 E_{0}} \mathcal{J}_{x}, \\
& \chi_{z}=\oint\left[b_{z}(s)+\eta b_{\delta x}(s)\right] d s \equiv \frac{U_{0}}{2 E_{0}}(2+\mathcal{D}) \equiv \frac{U_{0}}{2 E_{0}} \mathcal{J}_{z},
\end{aligned}
$$

where $U_{0}=\oint d s P_{\gamma}$ is the total radiated energy per turn and we have introduced the standard notation of $\mathcal{D}, \mathcal{J}_{x}$, and $\mathcal{J}_{z}$ for the damping partition numbers. This is a wellknown result in [1], and a similar rederivation has been given in $[10,11]$. Note that one can think of dispersion as causing coupling, and that the local and global sum rules of Eqs. (55) and (56) are satisfied.

\section{J. Uncoupled equilibrium emittances (Sands results)}

Now that we have the global diffusion coefficients and damping decrements for the uncoupled case, Eq. (47) gives the equilibrium values of the invariants, or in terms of the emittances $\epsilon_{a}=\left\langle g_{a}\right\rangle / 2$ we find

$$
\begin{gathered}
\epsilon_{x}=\frac{(55 / 48 \sqrt{3}) \alpha_{0} \gamma^{5} \oint d s \frac{\mathcal{H}_{x}}{\left|\rho^{3}\right|}}{\frac{2 U_{0}}{E_{0}} \mathcal{J}_{x}}, \\
\epsilon_{z}=\frac{(55 / 48 \sqrt{3}) \alpha_{0} \gamma^{5} \frac{a}{\mu_{s}} \oint d s \frac{1}{\left|\rho^{3}\right|}}{\frac{2 U_{0}}{E_{0}} \mathcal{J}_{z}} .
\end{gathered}
$$

\section{PERTURBATION THEORY NEAR A RESONANCE}

The global damping and diffusion coefficients are constructed from the eigenvectors of the one-turn map, $\boldsymbol{v}_{k}(s)$, along with the damping and diffusion matrices $B(s)$ and $D(s)$. In this section we use perturbation theory to find approximate expressions for the $v_{k}$ 's. We will construct invariants and damping and diffusion coefficients from these eigenvectors in the following section. In this section, we consider the 4D phase space of horizontal-longitudinal synchrobetatron dynamics, ignoring the vertical dimension.

\section{A. Adding a perturbation}

In Sec. II, we discussed the uncoupled storage ring. We now introduce some additional coupling element at some position in the ring. This changes the one-turn map, and as discussed in Sec. II, we could eigenanalyze the new ring and find the new equilibrium beam distribution. What we really need are the new eigenvectors. Assuming the coupling is small, we would like to do perturbation theory to find them. However, doing perturbation theory directly on $M_{\text {uncoupled }}$ is problematic near a resonance. At the degen- eracy, the eigenvectors are also degenerate, meaning that linear combinations of eigenvectors are still eigenvectors. One must add a perturbation to break the degeneracy and pick out specific eigenvectors. ${ }^{10}$ The degeneracy is broken by two relevant small quantities. First, there is the distance in tune space from resonance, and second, there is the coupling. Interesting perturbative effects occur when these two quantities are of similar size. The correct lowest order eigenvectors are not necessarily those of $M_{\text {uncoupled }}$. We will thus consider $M_{\text {uncoupled }}$ to be $M_{0}+M_{1 \mu}$ where $M_{0}$ is the map exactly on resonance. Instead of doing perturbation theory on $M_{\text {uncoupled }}$, we will do it on $M_{0}$. If we turn off the coupling, we will of course expect to get the correct eigenvalues and eigenvectors for $M_{\text {uncoupled }}$.

There are many ways to define such an on-resonance $M_{0}$. We choose to do so in such a way that the eigenvectors remain to be given by Eq. (92). This can be accomplished by choosing

$$
M_{0}=\left(\begin{array}{cc}
M_{x 0} & 0 \\
0 & M_{z 0}
\end{array}\right)
$$

where

$$
M_{x 0}=e^{\mu_{x 0} J_{x}}, \quad M_{z 0}=e^{\mu_{z 0} J_{z}},
$$

where $\mu_{x 0}$ and $\mu_{z 0}$ are their degenerate (exactly on resonance) values and $J_{x}$ and $J_{z}$ are defined as before, i.e., by (82) and (83). In particular, $\alpha_{x, z}, \beta_{x, z}$, and $\gamma_{x, z}$ are the uncoupled lattice functions derived from $M_{\text {uncoupled. }}$ Let us write ${ }^{11} \mu_{x}=\mu_{x 0}+\delta \mu_{x} / 2$ and $\mu_{z}=\mu_{z 0}+\delta \mu_{z} / 2$, then the perturbation to $M_{0}$ is given to lowest order as

$$
\begin{aligned}
M_{1 \mu} & =\left(\begin{array}{cc}
\frac{\delta \mu_{x}}{2} J_{x} M_{x 0} & 0 \\
0 & \frac{\delta \mu_{z}}{2} J_{z} M_{z 0}
\end{array}\right) \\
& =\left(\begin{array}{cc}
\frac{\delta \mu_{x}}{2} J_{x} & 0 \\
0 & \frac{\delta \mu_{z}}{2} J_{z}
\end{array}\right) M_{0} .
\end{aligned}
$$

For example, in the case of the sum resonance, $\mu_{x}+\mu_{z}=$ $\delta \mu$ where $\delta \mu$ is small [recall the redefinition Eq. (84)]. We then write $\mu_{x 0}=\mu, \mu_{z 0}=-\mu$, and $\delta \mu_{x}=\delta \mu_{z}=\delta \mu$. For the difference resonance, $\mu_{x}-\mu_{z}=\delta \mu$, and we have $\mu_{x 0}=\mu_{z 0}=\mu$ and $\delta \mu_{x}=-\delta \mu_{z}=\delta \mu$. For the integer resonance in $\mu_{x}$, we have $\mu_{x 0}=\mu_{-x 0}=0$ and $\delta \mu_{x}=$ $2 \mu_{x}$. In this case $\mu_{z 0}$ can be anything; although in practice, the synchrotron tune will typically be small. Finally, for a half-integer resonance in $\mu_{x}$, we have $\mu_{x 0}=\pi$, and $\delta \mu_{x}=2\left(\mu_{x}-\pi\right)$. Along with the corresponding $\mu_{z}$ in-

\footnotetext{
${ }^{10}$ For more on this point, see a discussion of degenerate perturbation theory in quantum mechanics, such as in [20], pp. 227-231, as well as our derivations in the next section and in Appendix B.

${ }^{11}$ The factor of $1 / 2$ is for later convenience-it allows for the interpretation of $\delta \mu_{x, z}$ as the splitting between the uncoupled nearly degenerate phase advances.
} 
teger and half-integer resonances, this covers all the single linear resonances.

The matrix $M_{1 \mu}$ specifies the deviation of the uncoupled map from being exactly on resonance. In addition to the perturbation $M_{1 \mu}$, we still have to add the perturbation coming from the coupling. We designate its contribution to the one-turn map as $M_{1 \xi}$. This could come from an error in the lattice, or from an added coupling element (such as a crab cavity) somewhere in the ring, or in the case of dispersion at an rf cavity, which can be considered to be intrinsically coupling (not due to errors). We show how to find $M_{1 \xi}$ for a dispersive rf cavity and a crab cavity in a later section. We write

$$
M=M_{0}+M_{1}, \quad M_{1}=M_{1 \mu}+M_{1 \xi} .
$$

As mentioned, $M_{1 \mu}$ does not change the eigenvectors of $M_{0} ; M_{1 \xi}$ will, however. To be more explicit about the perturbation $M_{1 \xi}$, suppose that at position $s_{j}$ we insert a perturbation $1+P\left(s_{j}\right)$. Let there be $n$ such perturbations, arranged such that $0<s_{1}<s_{2}<\cdots<s_{n}<C$. We have defined the observation position to be $s=0$. The new oneturn map at the observation position is

$$
M=T_{s_{n} \rightarrow C}\left[1+P\left(s_{n}\right)\right] \ldots T_{s_{1} \rightarrow s_{2}}\left[1+P\left(s_{1}\right)\right] T_{0 \rightarrow s_{1}} .
$$

Keeping lowest order in the perturbations, we find

$$
M_{1 \xi}=\left(\sum_{m=1}^{n} T_{s_{m} \rightarrow C} P\left(s_{m}\right) T_{0 \rightarrow s_{m}}^{-1}\right) M_{\text {uncoupled }}(0) .
$$

\section{B. Perturbation to eigenvalues and eigenvectors}

Because $M_{0}$ is degenerate, we use degenerate perturbation theory to find the perturbed eigenvectors and eigenvalues. We here give a brief derivation of the results we will need for most of what follows. The discussion is carried out in greater generality and detail in Appendix B.

Consider specific values of $j$ and $k$ such that a degeneracy occurs with $\mu_{j 0}=\mu_{k 0}=\mu_{0}$. Then $v_{j 0}$ and $v_{k 0}$ form a degenerate subspace of $M_{0}$; that is, any linear combinations of $v_{j 0}$ and $v_{k 0}$ are still degenerate eigenvectors of $M_{0}$. The perturbation will pick out a particular linear combination in addition to perturbing it, thus breaking the degeneracy. We write this as

$$
\boldsymbol{v}_{j}=\tilde{\boldsymbol{v}}_{j 0}+\tilde{\boldsymbol{v}}_{j 1}+\ldots, \quad \boldsymbol{v}_{k}=\tilde{\boldsymbol{v}}_{k 0}+\tilde{\boldsymbol{v}}_{k 1}+\ldots,
$$

where $\tilde{v}_{j 0}$ and $\tilde{v}_{k 0}$ are the 0th order linear combinations:

$$
\tilde{\boldsymbol{v}}_{j 0}=c_{j 0}^{j} \boldsymbol{v}_{j 0}+c_{k 0}^{j} \boldsymbol{v}_{k 0}, \quad \tilde{\boldsymbol{v}}_{k 0}=c_{j 0}^{k} \boldsymbol{v}_{j 0}+c_{k 0}^{k} \boldsymbol{v}_{k 0},
$$

where $v_{j 0}$ and $v_{k 0}$ are the eigenvectors of $M_{0}$ with eigenvalues $\lambda_{j 0}$ and $\lambda_{k 0}$, and are identified with two of the four candidates $v_{x}, v_{-x}, \boldsymbol{v}_{z}$, and $\boldsymbol{v}_{-z}$ from Eq. (92) as the case may be.
We then expand the eigenvalues up to 1st order,

$$
\lambda_{j}=\lambda_{j 0}+\lambda_{j 1}+\ldots, \quad \lambda_{k}=\lambda_{k 0}+\lambda_{k 1}+\ldots
$$

The eigenvalue equations up to 1 st order for $v_{j}$ and $v_{k}$ say

$$
\left(M_{0}+M_{1}\right)\left(\tilde{v}_{j 0}+\tilde{v}_{j 1}\right)=\left(\lambda_{j 0}+\lambda_{j 1}\right)\left(\tilde{v}_{j 0}+\tilde{v}_{j 1}\right),
$$$$
\left(M_{0}+M_{1}\right)\left(\tilde{v}_{k 0}+\tilde{v}_{k 1}\right)=\left(\lambda_{k 0}+\lambda_{k 1}\right)\left(\tilde{v}_{k 0}+\tilde{v}_{k 1}\right) \text {. }
$$

We now multiply by $\boldsymbol{v}^{j 0}$ and $\boldsymbol{v}^{k 0}$ on the left to each of these equations. We again keep up to first order. After using the 0th order eigenvalue equation, and using the expansion in Eq. (119), we find the eigenvalue equation

$$
\left(\begin{array}{ll}
\mathcal{M}_{j j} & \mathcal{M}_{j k} \\
\mathcal{M}_{k j} & \mathcal{M}_{k k}
\end{array}\right)\left(\begin{array}{c}
c_{j 0}^{j, k} \\
c_{k 0}^{j, k}
\end{array}\right)=\lambda_{j, k 1}\left(\begin{array}{c}
c_{j 0}^{j, k} \\
c_{k 0}^{j, k}
\end{array}\right),
$$

where we have defined

$$
\mathcal{M}_{m n}=v^{m 0} M_{1} v_{n 0}
$$

Thus, we see that in order to find the coefficients for the coupled eigenvectors, we need to find the eigenvectors of this perturbation matrix $\mathcal{M}$, whereas to find the perturbation to the eigenvalues, we find its eigenvalues.

The matrix elements of $\mathcal{M}$ are mutually interrelated because $M_{1}$ is not arbitrary but must be such that $M$ is symplectic. We write $M=M_{0}+M_{1}$ and apply the symplectic condition (12). Given that $M_{0}$ is symplectic, we find, to lowest order, ${ }^{12}$

$$
M_{0}^{T}\left(J M_{1}\right)=\left(J M_{1}\right)^{T} M_{0} .
$$

which says that $M_{0}^{T} J M_{1}$ is a symmetric matrix. Taking a transpose and conjugate of Eq. (123), and using this equation and the definition (16), we can now prove the following relationships among the matrix elements $\mathcal{M}_{m n}$ $(m, n= \pm 1, \pm 2)$ :

$$
\begin{gathered}
\mathcal{M}_{m n}=-\operatorname{sgn}(m) \operatorname{sgn}(n) \lambda_{m 0} \lambda_{n 0} \mathcal{M}_{n m}^{*}, \\
\mathcal{M}_{m n}=\mathcal{M}_{-m-n}^{*} .
\end{gathered}
$$

For later convenience, we would also like to define some additional related quantities. As we saw in Eqs. (114) and (117), it is often convenient to write $M_{1}$ in the form

$$
M_{1}=P M_{0} .
$$

We then define

$$
r_{m n}=v^{m 0} P v_{n 0}
$$

\footnotetext{
${ }^{12}$ In the case that $M_{1}$ is proportional to some parameter and that $M$ is symplectic for some continuous range of that parameter, then Eq. (124) will be true exactly, not just to lowest order, which one sees by writing out the symplectic condition as a Taylor series in this parameter and setting terms of the same order equal to each other. This situation applies to the cases considered in this paper. For the rf cavity, this parameter is $r$, and for the crab cavity it is $\xi_{c}$, see Eqs. (205) and (226).
} 
from which follows that

$$
r_{m n}=e^{-i \mu_{n 0}} \mathcal{M}_{m n} .
$$

In terms of the $r_{m n}$, the relations (125) and (126) state

$$
\begin{gathered}
r_{m n}=-\operatorname{sgn}(m) \operatorname{sgn}(n) r_{n m}^{*}, \\
r_{m n}=r_{-m-n}^{*} .
\end{gathered}
$$

We now solve the eigenvalue Eq. (122). In terms of the $r_{m n}$, the eigenvalues are

$$
\begin{aligned}
& \lambda_{j 1}=\frac{e^{i \mu_{0}}}{2}\left[\left(r_{j j}+r_{k k}\right)+\left(r_{j j}-r_{k k}\right) \sqrt{1+\frac{4 r_{j k} r_{k j}}{\left(r_{j j}-r_{k k}\right)^{2}}}\right], \\
& \lambda_{k 1}=\frac{e^{i \mu_{0}}}{2}\left[\left(r_{j j}+r_{k k}\right)-\left(r_{j j}-r_{k k}\right) \sqrt{1+\frac{4 r_{j k} r_{k j}}{\left(r_{j j}-r_{k k}\right)^{2}}}\right] .
\end{aligned}
$$

From the perturbations to the eigenvalues, we can find the perturbation to the phase advances. To this end, we write

$$
\begin{aligned}
& \lambda_{j}=\lambda_{j 0}+\lambda_{j 1}=e^{i\left(\mu_{0}+\mu_{j 1}\right)} \approx e^{i \mu_{0}}\left(1+i \mu_{j 1}\right), \\
& \lambda_{k}=\lambda_{k 0}+\lambda_{k 1}=e^{i\left(\mu_{0}+\mu_{k 1}\right)} \approx e^{i \mu_{0}}\left(1+i \mu_{k 1}\right) .
\end{aligned}
$$

from which we see that the first order perturbation to the phase advances are given by

$$
\mu_{j 1}=-i e^{-i \mu_{0}} \lambda_{j 1}, \quad \mu_{k 1}=-i e^{-i \mu_{0}} \lambda_{k 1} .
$$

Let us decompose the shift in the phase advances into an average shift and difference from that average; i.e., we write

$$
\mu_{j 1}=\bar{\mu}+\frac{\delta \mu_{1}}{2}, \quad \mu_{k 1}=\bar{\mu}-\frac{\delta \mu_{1}}{2},
$$

which implies

$$
\bar{\mu}=\frac{1}{2}\left(\mu_{j 1}+\mu_{k 1}\right), \quad \delta \mu_{1}=\mu_{j 1}-\mu_{k 1} .
$$

Using (132), (134), and (136), we find

$$
\begin{gathered}
\bar{\mu}=-i\left(r_{j j}+r_{k k}\right), \\
\delta \mu_{1}=-\frac{i}{2}\left(r_{j j}-r_{k k}\right) \sqrt{1+\frac{4 r_{j k} r_{k j}}{\left(r_{j j}-r_{k k}\right)^{2}}} .
\end{gathered}
$$

Now, using Eq. (130), $r_{j j}$ and $r_{k k}$ are purely imaginary. We can then define the quantity

$$
\Delta \mu=-i\left(r_{j j}-r_{k k}\right),
$$

and we know that it will be real. In fact, it is useful to focus on this term a bit more for the moment. Recall that $M_{1}=$ $M_{1 \mu}+M_{1 \xi}$. We correspondingly write $r_{m n}=r_{m n \mu}+$ $r_{m n \xi}$ and $\Delta \mu=\Delta \mu_{\mu}+\Delta \mu_{\xi}$ for the two corresponding parts of $r_{m n}$ and $\Delta \mu$. The form of $M_{1 \mu}$ was given in Eq. (114). Note that $M_{0}$ factored out so that the perturba- tion coming from the difference from resonance, $P_{\mu}$ is

$$
P_{\mu}=\left(\begin{array}{cc}
\delta \mu_{x} J_{x} & 0 \\
0 & \delta \mu_{z} J_{z}
\end{array}\right)
$$

Now, one can easily show that (ignoring the 0 's in the $z$ and $\delta$ components) $v_{x}$ is an eigenvector of $J_{x}$ with eigenvalue $i$, and $v_{-x}$ with eigenvalue $-i$. Likewise for $v_{z}$ and $v_{-z}$. Using this, we immediately get that $r_{11 \mu}=-r_{-1-1 \mu}=$ $i \delta \mu_{x}$ and $r_{22 \mu}=r_{-2-2 \mu}=i \delta \mu_{z}$. We have given the expressions for $\delta \mu_{x, z}$ in the paragraph following Eq. (114). The result is that $\Delta \mu_{\mu}$ is $\delta \mu=\mu_{x}-\mu_{z}$ for the difference resonance, $\delta \mu=\mu_{x}+\mu_{z}$ for the sum resonance, and $2 \mu_{x}$ or $2\left(\mu_{x}-\pi\right)$ for an $x$ integer or half-integer resonance, respectively, and likewise for $z$. Then we can write

$$
\Delta \mu=\delta \mu-i\left(r_{j j \xi}-r_{k k \xi}\right) .
$$

$\Delta \mu$ will be one of the main parameters we need to compute in any given example. We refer to it as the "splitting parameter." The additional term involving the perturbation gives the direct change to the tune split (times $2 \pi$ ) assuming $r_{j k \xi}=0$.

Next, using Eq. (130), we find

$$
r_{j k} r_{k j}=-\operatorname{sgn}(j) \operatorname{sgn}(k)\left|r_{j k}\right|^{2} .
$$

If we then define

$$
\xi=2\left|r_{j k}\right|
$$

Equation (138) becomes

$$
\delta \mu_{1}=\frac{\Delta \mu}{2} \sqrt{1+\operatorname{sgn}(j) \operatorname{sgn}(k) \frac{\xi^{2}}{\Delta \mu^{2}}} .
$$

In the case where one mode is positive and the other negative, we see that $\delta \mu_{1}$ becomes imaginary and hence we have an instability for

$$
\xi>|\Delta \mu|
$$

whereas if both modes are positive or both negative, there is no instability. It is here that we see why the difference resonance is always stable, whereas the sum, integer, and half-integer resonances can have instabilities. In the case of the difference resonance, the positive $x$ mode is degenerate with the positive $z$ mode and likewise for the negative modes. For the sum resonance, the positive $x$ mode is degenerate with the negative $z$ mode and the negative $x$ mode degenerate with the positive $z$ mode. We can also understand the instability of the integer and half-integer resonances. In the case of the $x$ integer and half-integer resonances, the positive $x$ mode is degenerate with the negative $x$ mode, whereas for the $z$ integer and half-integer resonances, the positive and negative $z$ modes are degenerate. So, the difference resonance has degenerate modes of the same sign and hence stability, while the sum, integer and half-integer resonances have degenerate modes of opposite signs and hence the possibility of instability. 
Next, consider the eigenvectors of $\mathcal{M}$. These can be written as

$$
\begin{aligned}
& \left(\begin{array}{c}
c_{j 0}^{j} \\
c_{k 0}^{j}
\end{array}\right)=\left(\frac{r_{j j}-r_{k k}}{2 r_{k j}}\left[1+\sqrt{1+\frac{4 r_{j k} r_{k j}}{\left(r_{j j}-r_{k k}\right)^{2}}}\right]\right), \\
& \left(\begin{array}{c}
c_{j 0}^{k} \\
c_{k 0}^{k}
\end{array}\right)=\left(\frac{r_{j j}-r_{k k}}{2 r_{k j}}\left[1-\sqrt{1+\frac{4 r_{j k} r_{k j}}{\left(r_{j j}-r_{k k}\right)^{2}}}\right]\right) .
\end{aligned}
$$

These eigenvectors have yet to be normalized. All of the quantities here fit nicely into the definitions we have already made except for the $r_{k j}$ in the denominator. We know that it has an absolute value of $\frac{\xi}{2}$, but it also has an additional phase. Let us thus define a phase $\phi$ by

$$
r_{j k}=\frac{\xi}{2} e^{i \phi}
$$

or

$$
\phi=\arg \left(r_{j k}\right),
$$

where $\arg ()$ means taking the angle in the complex plane with $\phi \in[-\pi, \pi]$. We use (130) to relate $r_{j k}$ to $r_{k j}$, noting that we get an additional minus sign when $j$ and $k$ have the same sign. The eigenvectors look different depending on whether the modes have the same or different signs. When both modes have the same sign, after normalization, we can express the eigenvectors as

$$
\begin{aligned}
& \tilde{\boldsymbol{v}}_{j 0}=\cos \frac{\theta}{2} \boldsymbol{v}_{j 0}+i e^{-i \phi} \sin \frac{\theta}{2} \boldsymbol{v}_{k 0}, \\
& \tilde{\boldsymbol{v}}_{k 0}=i e^{i \phi} \sin \frac{\theta}{2} \boldsymbol{v}_{j 0}+\cos \frac{\theta}{2} \boldsymbol{v}_{k 0},
\end{aligned}
$$

where

$$
\tan \theta=\frac{\xi}{\Delta \mu} .
$$

When the modes have opposite signs and supposing that $j$ is the positive mode, we find the normalized eigenvectors to be

$$
\begin{aligned}
& \tilde{\boldsymbol{v}}_{j 0}=\cosh \frac{\theta}{2} \boldsymbol{v}_{j 0}-i e^{-i \phi} \sinh \frac{\theta}{2} \boldsymbol{v}_{k 0}, \\
& \tilde{\boldsymbol{v}}_{k 0}=i e^{i \phi} \sinh \frac{\theta}{2} \boldsymbol{v}_{j 0}+\cosh \frac{\theta}{2} \boldsymbol{v}_{k 0},
\end{aligned}
$$

where

$$
\tanh \theta=\frac{\xi}{\Delta \mu} .
$$

We have normalized these eigenvectors such that

$$
\tilde{\boldsymbol{v}}^{j 0} \tilde{\boldsymbol{v}}_{k 0}=\delta_{j k} \text {. }
$$

The overall phases are chosen so that for $\theta=0, \tilde{v}_{j 0}=v_{j 0}$ and $\tilde{\boldsymbol{v}}_{k 0}=\boldsymbol{v}_{k 0}$. Note also that for this choice of overall phase, for the cases when $k=-j$, i.e., the integer or halfinteger resonance, the condition that $\tilde{v}_{j 0}=i \tilde{v}_{k 0}^{*}$ is satisfied.

\section{Cases of resonances}

We now consider the specific cases for values of $j$ and $k$. We will first consider the integer and half-integer resonances and then the sum and difference resonances. The integer and half-integer resonances involve a single pair of degenerate eigenvalues: for the integer resonance, an eigenvalue pair approaches the positive real axis while for the half-integer resonance, a pair approaches the negative real axis. In the cases of the sum and difference resonances, both eigenvalue pairs become degenerate.

\section{Integer/half-integer resonance}

The integer/half-integer resonances are covered by the cases where $(j, k)$ equals $(1,-1)$ or $(2,-2)$. Let us consider the case $(j, k)=(2,-2)$. This is the case of an integer or half-integer resonance for $\mu_{z}$. For the integer resonance, we have $\mu_{z 0}=\mu_{-z 0}=0$ and for the half-integer resonance, we have $\mu_{z 0}=-\mu_{-z 0}=\pi$. Here, the perturbation matrix is

$$
\left(\begin{array}{ll}
\mathcal{M}_{22} & \mathcal{M}_{2-2} \\
\mathcal{M}_{-22} & \mathcal{M}_{-2-2}
\end{array}\right)
$$

The coupling parameter is given by

$$
\xi=2\left|r_{2-2}\right|,
$$

The splitting parameter $\Delta \mu$ is given by

$$
\Delta \mu=2 \mu_{z}-2 i r_{22 \xi}
$$

for the integer resonance and

$$
\Delta \mu=2\left(\mu_{z}-\pi\right)-2 i r_{22 \xi},
$$

for the half-integer resonance. The average shift in the phase advances of $\mu_{ \pm z}, \bar{\mu}$, is given by

$$
\bar{\mu}=-i\left(r_{22 \xi}+r_{-2-2 \xi}\right)=2 \operatorname{Re}\left(r_{22 \xi}\right)=0,
$$

where we have used (130) and (131). This result is expected due to the fact that $\mu_{2}=-\mu_{-2}$. In terms of these quantities, then, we can express the perturbed $x$ eigenphase advance to first order as

$$
\mu_{2}=\frac{\Delta \mu}{2} \sqrt{1-\frac{\xi^{2}}{\Delta \mu^{2}}}
$$

for the integer resonance and

$$
\mu_{2}=\pi+\frac{\Delta \mu}{2} \sqrt{1-\frac{\xi^{2}}{\Delta \mu^{2}}} .
$$

for the half-integer resonance. Note that these reduce to $\mu_{z}$ when the perturbation is turned off. The coupling angle is defined by 


$$
\tanh \theta=\frac{\xi}{\Delta \mu}
$$

so that $\theta$ ranges from $-\infty$ to $\infty$ with the sign determined by the sign of $\Delta \mu$.

In terms of this angle, we can express the eigenphase advance as

$$
\mu_{2}=\frac{\Delta \mu}{2} \operatorname{sech}(\theta)
$$

for the integer resonance, with $\Delta \mu$ given in (156), or

$$
\mu_{2}-\pi=\frac{\Delta \mu}{2} \operatorname{sech}(\theta)
$$

for the half-integer resonance, with $\Delta \mu$ given in (157). Note that as $\theta$ gets large, the eigenphase advance goes to 0 . At the instability, $\theta$ passes through infinity and $\mu_{2}$ becomes complex. by

Finally, the relative phase for the eigenvectors is given

$$
\phi=\arg \left(r_{2-2}\right)
$$

for both the integer and half-integer resonance. Equation (151) then gives the eigenvectors in terms of these quantities as

$$
\begin{aligned}
\tilde{v}_{2} & =\cosh \frac{\theta}{2} v_{z}-i e^{-i \phi} \sinh \frac{\theta}{2} v_{-z}, \\
\tilde{v}_{-2} & =i e^{i \phi} \sinh \frac{\theta}{2} v_{z}+\cosh \frac{\theta}{2} v_{-z} .
\end{aligned}
$$

We can write these explicitly as

$$
\begin{aligned}
v_{2}= & \frac{1}{\sqrt{2}} \\
& \times\left(\begin{array}{c}
\sqrt{\beta_{z}}\left[\cosh \left(\frac{\theta}{2}\right)-e^{-i \phi} \sinh \left(\frac{\theta}{2}\right)\right] \\
\frac{1}{\beta_{z}}\left[\left(i-\alpha_{z}\right) \cosh \left(\frac{\theta}{2}\right)+\left(i+\alpha_{z}\right) e^{-i \phi} \sinh \left(\frac{\theta}{2}\right)\right] \\
0 \\
0
\end{array}\right)
\end{aligned}
$$

and $v_{-2}=-i v_{2}^{*}$. Note that only the 2D $z$ phase space components of the eigenvectors get mixed together by the perturbation. Further, only this subspace of the perturbation matrix enters into the coupling angle and phase, as is evident in (154). Thus, this is a fully $2 \mathrm{D}$ calculation. One might wonder what happened to the coupling, which is a 4D phenomena. It turns out that we have in fact missed the coupling, and that to include it appropriately, we need to go to second order perturbation theory. We find that the eigenvectors are still given by (165), but now the expressions for the coupling angle and phase includes the full 4D coupling perturbation matrix. We discuss this further in Sec. III E.
For the case of the $\mu_{x}$ integer and half-integer resonances, the perturbation matrix is

$$
\left(\begin{array}{cc}
\mathcal{M}_{11} & \mathcal{M}_{1-1} \\
\mathcal{M}_{-11} & \mathcal{M}_{-1-1}
\end{array}\right)
$$

and the preceding results are repeated with 2 and $z$ replaced by 1 and $x$.

\section{Sum resonance}

Next we consider the sum resonance where we have the degeneracies $(j, k)=(1,-2)$ and $(j, k)=(-1,2)$. Note that both of these degeneracies must occur together because of symplectity of $M$. The perturbation matrices are

$$
\left(\begin{array}{cc}
\mathcal{M}_{11} & \mathcal{M}_{1-2} \\
\mathcal{M}_{-21} & \mathcal{M}_{-2-2}
\end{array}\right), \quad\left(\begin{array}{cc}
\mathcal{M}_{-1-1} & \mathcal{M}_{-12} \\
\mathcal{M}_{2-1} & \mathcal{M}_{22}
\end{array}\right) .
$$

We find a single coupling parameter

$$
\xi=2\left|r_{1-2}\right| \text {. }
$$

$\Delta \mu$ is given by

$$
\Delta \mu=\mu_{x}+\mu_{z}-i\left(r_{11 \xi}-r_{-2-2 \xi}\right) .
$$

The coupling angle is now defined by

$$
\tanh \theta=\frac{\xi}{\Delta \mu} .
$$

Next, $\bar{\mu}$ is given by

$$
\bar{\mu}=-i\left(r_{11 \xi}+r_{-2-2 \xi}\right) .
$$

The eigenphase advances are

$$
\begin{aligned}
& \mu_{1}=\mu+\bar{\mu}+\frac{\Delta \mu}{2} \operatorname{sech}(\theta), \\
& \mu_{2}=-\mu_{-2}=-\mu-\bar{\mu}+\frac{\Delta \mu}{2} \operatorname{sech}(\theta) .
\end{aligned}
$$

The phase for the eigenvectors is given by

$$
\phi=\arg \left(r_{1-2}\right) .
$$

The eigenvectors are then given in terms of these quantities as

$$
\begin{aligned}
\tilde{v}_{1} & =\cosh \frac{\theta}{2} v_{x}-i e^{-i \phi} \sinh \frac{\theta}{2} v_{-z}, \\
\tilde{v}_{-2} & =i e^{i \phi} \sinh \frac{\theta}{2} v_{x}+\cosh \frac{\theta}{2} v_{-z} .
\end{aligned}
$$

The other two eigenvectors are given by $v_{-1}=-i v_{1}^{*}$ and $v_{2}=-i v_{-2}^{*}$.

\section{Difference resonance}

For the difference resonance, we have the degeneracies $(1,2)$ and $(-1,-2)$. The perturbation matrices are

$$
\left(\begin{array}{ll}
\mathcal{M}_{11} & \mathcal{M}_{12} \\
\mathcal{M}_{21} & \mathcal{M}_{22}
\end{array}\right), \quad\left(\begin{array}{ll}
\mathcal{M}_{-1-1} & \mathcal{M}_{-1-2} \\
\mathcal{M}_{-2-1} & \mathcal{M}_{-2-2}
\end{array}\right) .
$$


We find a single coupling parameter

$$
\xi=2\left|r_{12}\right|
$$

$\Delta \mu$ is given by

$$
\Delta \mu=\mu_{x}-\mu_{z}-i\left(r_{11}-r_{22}\right) .
$$

The coupling angle is then given by

$$
\tan \theta=\frac{\xi}{\Delta \mu} .
$$

Here $\theta$ can range from $-\pi / 4$ to $\pi / 4$, again with $\Delta \mu$ determining the sign. The eigenphase advances are

$$
\begin{aligned}
& \mu_{1}=\mu+\bar{\mu}+\frac{\Delta \mu}{2} \sqrt{1+\frac{\xi^{2}}{\Delta \mu^{2}}}, \\
& \mu_{2}=\mu+\bar{\mu}-\frac{\Delta \mu}{2} \sqrt{1+\frac{\xi^{2}}{\Delta \mu^{2}}} .
\end{aligned}
$$

In terms of the coupling angle, we can express these as

$$
\begin{aligned}
& \mu_{1}=\mu+\bar{\mu}+\frac{\Delta \mu}{2} \sec (\theta), \\
& \mu_{2}=\mu+\bar{\mu}-\frac{\Delta \mu}{2} \sec (\theta) .
\end{aligned}
$$

The phase for the eigenvectors is given by

$$
\phi=\arg \left(r_{12}\right) .
$$

We can now construct the eigenvectors from (149)

$$
\begin{aligned}
& \tilde{v}_{1}=\cos \frac{\theta}{2} v_{x}+i e^{-i \phi} \sin \frac{\theta}{2} v_{z}, \\
& \tilde{v}_{2}=i e^{i \phi} \sin \frac{\theta}{2} v_{x}+\cos \frac{\theta}{2} v_{z} .
\end{aligned}
$$

The other two eigenvectors are $v_{-1}=-i v_{1}^{*}$ and $v_{-2}=$ $-i v_{2}^{*}$.

The results for $\xi, \Delta \mu, \phi$, and $\bar{\mu}$ for each of these resonances are summarized in the first six rows of Table I.

\section{Special case of an integer/half-integer resonance caused by coupling}

We have seen that there is a subtlety related to integer and half-integer resonances. One can see that the "true $x-z$ coupling" from $r_{12}$ or $r_{1-2}$ did not enter into the results. In fact, the integer/half-integer results we have derived follow from a strictly $2 \mathrm{D}$ phase space analysis, reproducing results already contained in [4]. However, we are also interested in the situation where the perturbation is strictly a coupling perturbation, i.e., $M_{1 \xi}$ only has elements in the two offdiagonal blocks. This will be the case for the perturbation due to a crab cavity when there is no dispersion at the cavity. We can then ask whether this coupling perturbation can cause an integer or half-integer resonance if it is strong enough. Clearly our first order perturbation theory is insufficient to answer this question, and we thus consider 2 nd order degenerate perturbation theory. We do this in Appendix B, providing an alternative derivation and further details in Appendix D. The result is that we can use the same results as above for the integer or half-integer resonance, except that we use as the perturbation matrix (consider the case when the resonance occurs in the $z$ dimension, i.e., $\mu_{z}$ is close to 0 or $\pi$ )

$$
\left(\begin{array}{cc}
\frac{\mathcal{M}_{12} \mathcal{M}_{21}}{\lambda_{20}-\lambda_{10}} & \frac{\mathcal{M}_{1-2} \mathcal{M}_{21}}{\lambda_{20}-\lambda_{10}} \\
+\frac{\mathcal{M}_{-12} \mathcal{M}_{2-1}}{\lambda_{20}-\lambda_{-10}} & +\frac{\mathcal{M}_{-12} \mathcal{M}_{2-1}}{\lambda_{20}-\lambda_{-10}} \\
+\mathcal{M}_{22} & +\mathcal{M}_{2-2} \\
\frac{\mathcal{M}_{12} \mathcal{M}_{-21}}{\lambda_{20}-\lambda_{10}} & \frac{\mathcal{M}_{1-2} \mathcal{M}_{-21}}{\lambda_{20}-\lambda_{10}} \\
+\frac{\mathcal{M}_{-12} \mathcal{M}_{-2-1}}{\lambda_{20}-\lambda_{-10}} & +\frac{\mathcal{M}_{-1-2} \mathcal{M}_{-2-1}}{\lambda_{20}-\lambda_{-10}} \\
+\mathcal{M}_{-22} & +\mathcal{M}_{-2-2}
\end{array}\right) .
$$

We note that we have here a mixture of first and second order quantities. Neglecting the second order quantities reduces this to Eq. (167).

For the case of the integer $z$ resonance, this matrix simplifies to

$$
\left(\begin{array}{cc}
\frac{\left|r_{12}\right|^{2}}{1-e^{-i \mu_{x}}}-\frac{\left|r_{-12}\right|^{2}}{1-e^{i \mu_{x}}} & i r_{21} r_{1-2} \cot \left(\frac{\mu_{x}}{2}\right)+r_{2-2} \\
+r_{22} & \frac{\left|r_{12}\right|^{2}}{1-e^{i \mu_{x}}}-\frac{\left|r_{-12}\right|^{2}}{1-e^{-i \mu_{x}}} \\
-i r_{21}^{*} r_{1-2}^{*} \cot \left(\frac{\mu_{x}}{2}\right)+r_{-22} & +r_{-2-2}
\end{array}\right)
$$

where the $r_{j k}$ here are really $r_{j k \xi}$, the matrix elements due to just the coupling perturbation. The perturbed eigenvalues and eigenvectors are now given by the eigenvalues and eigenvectors of this matrix. We can again express them in terms of a coupling parameter, splitting parameter and phase. The coupling parameter and phase are defined by

$$
\xi e^{i \phi}=\frac{1}{2}\left[r_{2-2}+i r_{21} r_{1-2} \cot \left(\frac{\mu_{x}}{2}\right)\right],
$$

which reduce to (155) and (164) if we ignore the second order term.

The splitting parameter is given by

$$
\Delta \mu=2 \mu_{z}-2 i r_{22 \xi}-\left(\left|r_{12}\right|^{2}+\left|r_{-12}\right|^{2}\right) \cot \left(\frac{\mu_{x}}{2}\right) \text {. }
$$

The results for the other integer and half-integer resonance are summarized in the last four rows of Table I. In terms of $\xi$ and $\Delta \mu$, we can again define a coupling angle

$$
\tanh \theta=\frac{\xi}{\Delta \mu}
$$

The eigenphase advance is now given by (162). It is useful, however, to write out the expression for $\mu_{2}$ more explicitly. Let us consider the case in which the perturbation is purely a coupling perturbation $\left(r_{22 \xi}=r_{2-2 \xi}=0\right)$ and in which $\left|r_{12}\right|=\left|r_{1-2}\right|{ }^{13}$ We then find

\footnotetext{
${ }^{13}$ See Appendix $\mathrm{C}$ for more on this condition.
} 
TABLE I. Linear resonances of a synchrobetatron coupled storage ring. For each of the linear resonances, we give general expressions for the quantities $\Delta \mu, \xi, \phi$, and $\bar{\mu}$. The quantities $r_{j k}$ here are $r_{j k \xi}$, i.e., the part of $r_{j k}$ coming from the coupling perturbation $P_{\xi}$. We have left off the subscript $\xi$ for convenience. The matrix elements $r_{j k \mu}$ coming from the difference from resonance are included explicitly in the $\Delta \mu$ and involve $\mu_{x}$ and $\mu_{z}$. The resonances with the abbreviation "Cp." refer to the full 2nd order calculation for the coupling-induced integer and half-integer resonances (see Appendix D).

\begin{tabular}{|c|c|c|c|c|c|}
\hline Reso. & Condition & $\Delta \mu(\bmod 2 \pi)$ & $\xi$ & $\phi$ & $\bar{\mu}$ \\
\hline Sum & $\mu_{x}+\mu_{z}=2 \pi n$ & $\mu_{x}+\mu_{z}-i\left(r_{11}-r_{-2-2}\right)$ & $2\left|r_{1-2}\right|$ & $\arg \left(r_{1-2}\right)$ & $-i\left(r_{11}+r_{-2-2}\right)$ \\
\hline Diff. & $\mu_{x}-\mu_{z}=2 \pi n$ & $\mu_{x}-\mu_{z}-i\left(r_{11}-r_{22}\right)$ & $2\left|r_{12}\right|$ & $\arg \left(r_{12}\right)$ & $-i\left(r_{11}+r_{22}\right)$ \\
\hline Iint $(x)$ & $\mu_{x}=2 \pi n$ & $2 \mu_{x}-2 i r_{11}$ & $2\left|r_{1-1}\right|$ & $\arg \left(r_{1-1}\right)$ & 0 \\
\hline Int $(z)$ & $\mu_{z}=2 \pi n$ & $2 \mu_{z}-2 i r_{22}$ & $2\left|r_{2-2}\right|$ & $\arg \left(r_{2-2}\right)$ & 0 \\
\hline$\frac{1}{2}$-int $(x)$ & $\mu_{x}=\pi(2 n+1)$ & $2\left(\mu_{x}-\pi\right)-2 i r_{11}$ & $2\left|r_{1-1}\right|$ & $\arg \left(r_{1-1}\right)$ & 0 \\
\hline$\frac{1}{2}$-int $(z)$ & $\mu_{z}=\pi(2 n+1)$ & $2\left(\mu_{z}-\pi\right)-2 i r_{22}$ & $2\left|r_{2-2}\right|$ & $\arg \left(r_{2-2}\right)$ & 0 \\
\hline Cp. int $(x)$ & $\mu_{x}=2 \pi n$ & $\begin{array}{c}2 \mu_{x}-2 i r_{11} \\
-\left(\left|r_{12}\right|^{2}+\left|r_{-12}\right|^{2}\right) \cot \left(\frac{\mu_{z}}{2}\right)\end{array}$ & $2\left|r_{1-1}+i r_{2-1} r_{12} \cot \left(\frac{\mu_{z}}{2}\right)\right|$ & $\arg \left(r_{1-1}+i r_{2-1} r_{12} \cot \left(\frac{\mu_{z}}{2}\right)\right)$ & 0 \\
\hline Cp. int $(z)$ & $\mu_{z}=2 \pi n$ & $\begin{array}{c}2 \mu_{z}-2 i r_{22} \\
-\left(\left|r_{12}\right|^{2}+\left|r_{-12}\right|^{2}\right) \cot \left(\frac{\mu_{x}}{2}\right)\end{array}$ & $2\left|r_{2-2}+i r_{1-2} r_{21} \cot \left(\frac{\mu_{x}}{2}\right)\right|$ & $\arg \left(r_{2-2}+i r_{1-2} r_{21} \cot \left(\frac{\mu_{x}}{2}\right)\right)$ & 0 \\
\hline Cp. $\frac{1}{2}$-int $(x)$ & $\mu_{x}=\pi(2 n+1)$ & $\begin{array}{c}2\left(\mu_{x}-\pi\right)-2 i r_{11} \\
+\left(\left|r_{12}\right|^{2}+\left|r_{-12}\right|^{2}\right) \tan \left(\frac{\mu_{z}}{2}\right)\end{array}$ & $2\left|r_{1-1}-i r_{2-1} r_{12} \tan \left(\frac{\mu_{z}}{2}\right)\right|$ & $\arg \left(r_{1-1}-i r_{1-2} r_{21} \tan \left(\frac{\mu_{z}}{2}\right)\right)$ & 0 \\
\hline Cp. $\frac{1}{2}$-int $(z)$ & $\mu_{z}=\pi(2 n+1)$ & $\begin{array}{c}2\left(\mu_{z}-\pi\right)-2 i r_{22} \\
+\left(\left|r_{12}\right|^{2}+\left|r_{-12}\right|^{2}\right) \tan \left(\frac{\mu_{x}}{2}\right)\end{array}$ & $2\left|r_{2-2}-i r_{1-2} r_{21} \tan \left(\frac{\mu_{x}}{2}\right)\right|$ & $\arg \left(r_{2-2}-i r_{1-2} r_{21} \tan \left(\frac{\mu_{x}}{2}\right)\right)$ & 0 \\
\hline
\end{tabular}

$$
\mu_{2}^{2}=\mu_{z}^{2}-\frac{1}{2} \mu_{z} \xi_{ \pm}^{2} \cot \left(\frac{\mu_{x}}{2}\right),
$$

where $\xi_{ \pm}=2\left|r_{12}\right|=2\left|r_{1-2}\right|$ is the coupling parameter for the sum or difference resonances, which we have assumed to be equal. We can now see that $\mu_{2}$ becomes unstable when

$$
\frac{1}{2} \xi_{ \pm}^{2} \cot \left(\frac{\mu_{x}}{2}\right)>\mu_{z}
$$

Applying a similar analysis, and under the same conditions, we find an instability near the half-integer $z$ resonance when

$$
\frac{1}{2} \xi_{ \pm}^{2} \tan \left(\frac{\mu_{x}}{2}\right)<\pi-\mu_{z}
$$

The results for the $x$ integer and half-integer resonances can be found by interchance of $x$ and $z$. Instabilities occur when

$$
\frac{1}{2} \xi_{ \pm}^{2} \cot \left(\frac{\mu_{z}}{2}\right)>\mu_{x}
$$

or

$$
\frac{1}{2} \xi_{ \pm}^{2} \tan \left(\frac{\mu_{z}}{2}\right)<\pi-\mu_{x}
$$

The general expressions for $\mu_{1}$ and $\mu_{2}$ are given in Eqs. (D23) and (D24) but much of the basic physics can be seen by examining these results. Note how the coupling parameters for the sum or difference resonance enter into all of these expressions along with the value of the other phase advance not going unstable. Since $\mu_{z}$ is negative (above transition), we see that for the $z$ integer resonance, there can only be an instability when $\nu_{x}>\frac{1}{2}$. For the crab cavity, this is indeed an important resonance to consider because $\nu_{x}>\frac{1}{2}$ is a typical operation condition and for realistic parameters, a storage ring can approach the instability region. For dispersion at an rf cavity, we will find that because of the form of $\xi_{ \pm}$(in particular, its dependence on the synchrotron tune), (190) is never satisfied and there is no such integer resonance.

\section{E. Evolution of eigenvectors around ring}

We have given expressions for the eigenvectors near each of the resonances, computed at the position of the perturbation. In order to find global quantities like equilibrium invariants, we will need to know how the eigenvectors evolve around the ring. We can determine this using Eq. (98).

Let us consider the integer or half-integer resonance. Take mode 2 for example. We have seen that we can write the perturbed eigenvector in the form

$$
v_{2}\left(s_{1}\right)=\cosh \left(\frac{\theta}{2}\right) v_{z}\left(s_{1}\right)-i e^{-i \phi} \sinh \left(\frac{\theta}{2}\right) v_{-z}\left(s_{1}\right)
$$

Applying $T_{12 \beta}$ to this vector we find

$$
\begin{aligned}
& v_{2}\left(s_{2}\right)=e^{i \psi_{z 12}} \cosh \left(\frac{\theta}{2}\right) v_{z}\left(s_{2}\right) \\
& -i e^{-i\left(\phi+\psi_{z 12}\right)} \sinh \left(\frac{\theta}{2}\right) v_{-z}\left(s_{2}\right) \text {. }
\end{aligned}
$$

We have used the fact that $\psi_{-z 12}=-\psi_{z 12}$. The overall phase does not enter into any physical quantities, so we can multiply by $e^{-i \psi_{z 12}}$ and we find that the new eigenvector is that of the old, but with the lattice functions advanced to the new values and the phase $\phi\left(s_{2}\right)$ related to $\phi\left(s_{1}\right)$ by 


$$
\phi\left(s_{2}\right)=\phi\left(s_{1}\right)+2 \psi_{z 12} .
$$

Likewise, for the $x$ integer/half-integer resonance, the phase in the definition of $v_{1}$ is

$$
\phi\left(s_{2}\right)=\phi\left(s_{1}\right)+2 \psi_{x 12} .
$$

For the difference resonance, we apply the same argument and find

$$
\phi\left(s_{2}\right)=\phi\left(s_{1}\right)+\psi_{x 12}-\psi_{z 12}
$$

For the sum resonance, we find

$$
\phi\left(s_{2}\right)=\phi\left(s_{1}\right)+\psi_{x 12}+\psi_{z 12} .
$$

The phase advances $\psi_{x 12}$ and $\psi_{z 12}$ are given in Eqs. (93) and (96).

In the following section we will use these near resonance eigenvectors we have derived to construct the local damping and diffusion coefficients which are then integrated around the ring. We have computed the eigenvectors at an arbitrary position $s_{2}$ by first computing them at the position of the perturbation $s_{1}$ and then transforming them to $s_{2}$ by applying $T_{12 \beta}$. For consistency, we would like to see that we get the same result if we do the perturbation theory directly on the one-turn map at $s_{2}$. Writing $M=$ $(1+P) M_{0}$ and transforming from position 1 to position 2 we find

$$
\begin{aligned}
{\left[1+P\left(s_{2}\right)\right] M_{0}\left(s_{2}\right) } & =M\left(s_{2}\right)=T_{12 \beta} M\left(s_{1}\right) T_{12 \beta}^{-1} \\
& =\left[1+T_{12 \beta} P\left(s_{1}\right) T_{12 \beta}^{-1}\right] M_{0}\left(s_{2}\right)
\end{aligned}
$$

so that

$$
P\left(s_{2}\right)=T_{12 \beta} P\left(s_{1}\right) T_{12 \beta}^{-1} .
$$

Now, applying this equation along with (98) to the definition of $r_{m n}$, (128), and using the symplectic property of $T_{12 \beta}$, we find that $r_{m n}$ transforms as

$$
r_{m n}\left(s_{2}\right)=e^{i\left(\psi_{n 12}-\psi_{m 12}\right)} r_{m n}\left(s_{1}\right),
$$

i.e., they are invariant except for an over phase change. From (202) we can see that $r_{j j}\left(s_{2}\right)=r_{j j}\left(s_{1}\right)$ and so all of the $\Delta \mu$ are global quantities. We can also show that the quantities $\xi$ are global quantities. For the sum, difference, and simple integer and half-integer resonances, it is obvious because of the absolute value. For the couplingcaused integer and half-integer and half-integer resonance, one must show that the two terms transform with the same phase. For example, using (202) several times on $\xi$ for the $x$ integer resonance (found in line 7 of Table I), we can show

$$
\xi\left(s_{2}\right)=\left|e^{-2 i \psi_{x 12}}\right| \xi\left(s_{1}\right)=\xi\left(s_{1}\right),
$$

and likewise for all the other $\xi$ 's. Thus, for each resonance, $\xi$ and $\Delta \mu$ are global quantities, independent of where they are calculated, and hence so is the coupling angle $\theta$. We can also use Eq. (202) to show that the expressions derived for $\phi(s)$ give the same results whether one first computes $\phi\left(s_{1}\right)$ and then uses $T_{12 \beta}$ to advance the eigenvectors to get $\phi\left(s_{2}\right)$, as we have done above, or if one first advances the $r_{m n}$ to $s_{2}$ and then computes the phase. The argument for the coupling-caused integer and half-integer resonances is the same as that used in (203). Finally, we note that near each resonance, the net change in $\phi$ is a multiple of $2 \pi$ to lowest order, and thus, modulo $2 \pi, \phi$ is a well-defined, periodic function in the ring.

To summarize, the coupling angle $\theta$ is a global quantity independent of position in the ring, whereas $\phi$ is a phase that changes locally, but is periodic around the ring, modulo $2 \pi$. The formulas for $\phi$ given in Table I refer to the value at the position of the perturbation. We will use the eigenvectors to find the local invariants, which will depend on $\phi$ through $\cos \phi$ and $\sin \phi$. To advance these quantities to another position, $\phi$ must be advanced using Eqs. (196)(199).

\section{EXAMPLES}

In the previous section we formulated a perturbation theory to find the eigenvectors and eigenvalues of the one-turn map near a linear resonance in the presence of a coupling perturbation. In this section we give two examples of synchrobetatron coupling perturbations and apply the foregoing results to these two cases. Our two examples are dispersion at an $\mathrm{rf}$ acceleration cavity and the addition of a crab cavity.

In these two examples, the perturbation occurs at a single location. The procedure for a given example is as follows. First we find the perturbation matrix $P_{\xi}$. From this quantity, we derive the $r_{j k \xi}$. Out of these quantities, we construct $\xi, \Delta \mu, \phi$, and $\bar{\mu}$ for each resonance. For the integer and half-integer resonances, we compute the full 2nd order coupled quantities given in the last four rows of Table I. However, we keep terms to the lowest order in the various small quantities such as $\mu_{s}$, the crab cavity strength $\xi_{c}$, or the dispersion at the rf cavity. In some cases, we will find that the naive first order calculation gives the same results; however, in others, the full calculation was required. In the case of the crab cavity, we do not assume that the dispersion at the crab cavity is particularly small, since it may be of some interest to know what the effect is of placing a crab cavity at an arbitrary position in the ring.

From $\xi, \Delta \mu$, and $\bar{\mu}$, we find the tune shift and the coupling angle $\theta$. From the tune shift, we can see whether or not an instability occurs. Out of $\theta$ and $\phi$, we construct the global damping and diffusion coefficients, dropping middle terms in all cases except for the $\mu_{z}=0$ integer resonance, as discussed previously. Once the equilibrium invariants are calculated, one could then use detailed knowledge of the lattice functions to find the beam distribution at a given position, using formulas for the beam distribution moments, $\Sigma$. 
For each resonance, we give explicit expressions for $\xi$, $\Delta \mu, \phi$, and $\bar{\mu}$, only constructing the other quantities when instructive.

\section{A. Dispersion at an rf cavity}

As our first example, we consider coupling due to dispersion at the rf cavity. The uncoupled longitudinal oneturn map $M_{z}$ was given by (88). This was derived in physical coordinates. If there is dispersion at the cavity, we must transform the map into betatron coordinates and (81) is no longer block diagonal. The cavity map is transformed with $\mathcal{B} T_{\text {cav }} \mathcal{B}^{-1}$. We would like to pull out the part that causes the synchrotron oscillations and consider the extra part as the perturbation. To do this, we write

$$
\mathcal{B} T_{\text {cav }} \mathcal{B}^{-1}=\left(1+P_{\text {rf }}\right) T_{\text {cav }},
$$

where $T_{\text {cav }}$ was given in (85), except here we work in the $4 \mathrm{D}$ space, so include a $2 \times 2$ identity matrix in the upper left block and 0's in the off-diagonal blocks. Using this prescription, we derive

$$
P_{\mathrm{rf}}=\frac{r}{a}\left(\begin{array}{cccc}
\eta \eta^{\prime} & -\eta^{2} & -\eta & 0 \\
\eta^{\prime 2} & -\eta \eta^{\prime} & -\eta^{\prime} & 0 \\
0 & 0 & 0 & 0 \\
-\eta^{\prime} & \eta & 0 & 0
\end{array}\right)
$$

From this matrix we construct the $r_{j k \xi}$ :

$$
\begin{aligned}
r_{11} & =-i \frac{r}{2 a} \mathcal{H}_{x}, \quad r_{1-1}=\frac{r}{2 a \beta_{x}}\left[\left(i+\alpha_{x}\right) \eta+\beta_{x} \eta^{\prime}\right]^{2}, \\
r_{12} & =i \frac{r}{2 a} \sqrt{\frac{\beta_{z}}{\beta_{x}}}\left[\left(i+\alpha_{x}\right) \eta+\beta_{x} \eta^{\prime}\right], \\
r_{1-2} & =-\frac{r}{2 a} \sqrt{\frac{\beta_{z}}{\beta_{x}}}\left[\left(i+\alpha_{x}\right) \eta+\beta_{x} \eta^{\prime}\right], \\
r_{22} & =r_{2-2}=0,
\end{aligned}
$$

where $\mathcal{H}_{x}$ is defined in Eq. (104). From these we find $\xi$, $\Delta \mu, \phi$, and $\bar{\mu}$ for each resonance out of which we can construct the perturbed eigenvalues and eigenvectors.

\section{Sum/difference resonance}

Examining the perturbation (205) we see that the determinant of the off-diagonal blocks is zero. From Appendix C, this tells us that the coupling parameters for the sum and difference resonances will be equal. Computing them, we find

$$
\xi_{ \pm}=\frac{r}{a} \sqrt{\mathcal{H}_{x} \beta_{z}}
$$

where the \pm indicates both the sum and difference resonance. If we expand this to lowest order in $\mu_{z}$, we find

$$
\xi_{ \pm}=\sqrt{\frac{\mathcal{H}_{x}}{a}}\left|\mu_{z}\right|^{3 / 2}
$$

Note that this goes to 0 at as $\mu_{z}$ goes to zero. This means that we expect the sum and difference resonances to become weaker for smaller synchrotron tune. For the splitting parameter we find

$$
\Delta \mu=\mu_{x}+\mu_{z}+\frac{r}{a} \mathcal{H}_{x}
$$

for the sum resonance and

$$
\Delta \mu=\mu_{x}-\mu_{z}+\frac{r}{a} \mathcal{H}_{x}
$$

for the difference resonance. The quantity $r$ is approximately $\mu_{z}^{2}$, so that term can typically be ignored. For both sum and difference resonance, the average shift of the eigenphase advance is

$$
\bar{\mu}=-\mu_{z}^{2} \frac{\mathcal{H}_{x}}{2 a} .
$$

The phase for the sum resonance is

$$
\phi=\arg \left[-\left(i+\alpha_{x}\right) \eta+\beta_{x} \eta^{\prime}\right]
$$

while for the difference resonance

$$
\phi=\arg \left[i\left(i+\alpha_{x}\right) \eta+\beta_{x} \eta^{\prime}\right] .
$$

To lowest order in $\mu_{z}$, the instability condition for the sum resonance is

$$
\sqrt{\frac{\mathcal{H}_{x}}{a}}\left|\mu_{z}\right|^{3 / 2}>\left|\mu_{x}+\mu_{z}\right| .
$$

\section{Integer/half-integer resonance}

We now consider the integer and half-integer resonances. Near the integer $x$ resonance, we find that the coupling parameter is proportional to $\mu_{z}^{4}$. There is still in fact an instability for $\nu_{x}$ near an integer, but it is extremely weak, with a width of order $\frac{\mathcal{H}_{x}}{a} \mu_{z}^{4}$. We do not consider this resonance further.

For the half-integer resonance in $\mu_{x}$, we find

$$
\xi=\mu_{z}^{2} \frac{\mathcal{H}_{x}}{a},
$$

$$
\begin{gathered}
\Delta \mu=2\left(\mu_{x}-\pi\right)-\frac{\mathcal{H} x}{a} \mu_{z}^{2}, \\
\phi=2 \arg \left(G_{x}+i \eta\right),
\end{gathered}
$$

where we define

$$
\mathcal{G}_{x}=\eta^{\prime} \beta_{x}+\eta \alpha_{x} .
$$

We find that there is an instability when

$$
\frac{\mathcal{H}_{x}}{a} \mu_{z}^{2}>\mu_{x}-\pi
$$

This says that there is an instability for $\mu_{x}>\pi$ with a width of $\frac{\mathcal{H}_{x}}{a} \mu_{z}^{2}$. Note that these results are identical to those we would obtain by using the 5 th row in Table I (first 
order calculation). The other terms are higher order in $\mu_{z}$ and have been ignored.

For the integer resonance in $\mu_{z}\left(\mu_{z}\right.$ near 0 , as is typically the case), we get

$$
\begin{gathered}
\xi=\left|\frac{\mu_{z}^{3} \mathcal{H}_{x}}{2 a} \cot \frac{\mu_{x}}{2}\right|, \\
\Delta \mu=2 \mu_{z}-\frac{\left|\mu_{z}\right|^{3} \mathcal{H}_{x}}{2 a} \cot \left(\frac{\mu_{x}}{2}\right), \\
\phi=0 .
\end{gathered}
$$

Here we find that $\mu_{2}$ is given by

$$
\mu_{2}=\mu_{z} \sqrt{1+\mu_{z}^{2} \frac{\mathcal{H}_{x}}{2 a} \cot \left(\frac{\mu_{x}}{2}\right)} .
$$

From this expression, we can see that there is no instability for the $\mu_{z}$ integer resonance. The quantity inside the square root could become negative only in two cases. One case is where the perturbation $P_{\mathrm{rf}}$ is not small and hence perturbation theory no longer applies. The other case is if $\mu_{x}$ is near 0 or $2 \pi$ in which case we are in the region of overlap between two different resonances in which we may need to consider the other resonances. Numerical calculation confirms that there is in fact no instability for small synchrotron tune, regardless of the values of the other parameters.

\section{B. Crab cavity}

As our second example, we consider a single crab cavity [21]. The map for the crab cavity is given by

$$
T_{\text {crab }}=\left(\begin{array}{cccc}
1 & 0 & 0 & 0 \\
0 & 1 & \xi_{c} & 0 \\
0 & 0 & 1 & 0 \\
\xi_{c} & 0 & 0 & 1
\end{array}\right)
$$

where $\xi_{c}$ gives the strength of the cavity. In the case where the crab cavity is used to correct for a half crossing angle $\Phi$ at the interaction point of a collider [3], assuming no resonance, $\xi_{c}$ is related to that crossing angle by

$$
\xi_{c}= \begin{cases}\frac{2 \Phi \sin \left(\pi \nu_{x}\right)}{\sqrt{\beta_{k} \beta_{x}^{*}}}, & \text { single crab cavity, } \\ \frac{\Phi}{\sqrt{\beta_{k} \beta_{x}^{*}}}, & \text { crab cavity pair, }\end{cases}
$$

where $\beta_{k}$ is the beta function at the crab cavity and $\beta_{x}^{*}$ is the beta function at the interaction point. For a pair of crab cavities, one positions them symmetrically about the interaction point such that there is a total of a $\pi$ phase shift between them and the second cancels out the effect of the first in the rest of the ring. The expression given here for a single crab cavity comes from computing the angle at the IP due to a change in closed orbit, assuming no resonance. Near a resonance, this formula needs to be reconsidered. Indeed, it is the purpose of this paper to determine the effect on beam dynamics due to coupling near a resonance.
We include this formula because in practice, the value of $\xi_{c}$ may be chosen assuming the machine is away from all resonances.

We here consider the case of a single crab cavity. Suppose we insert a crab cavity in the ring at position $s_{2}$. The rf cavity is assumed to be located at $s_{c}$ so that the partial momentum compaction factor as given in (87) involves integration from $s_{c}$ to $s_{2}$.

Transforming (224) into betatron coordinates, we get for the perturbation

$$
P_{\text {crab }}=\xi_{c}\left(\begin{array}{cccc}
-\eta & 0 & 0 & -\eta^{2} \\
-2 \eta^{\prime} & \eta & 1 & -\eta \eta^{\prime} \\
\eta \eta^{\prime} & -\eta^{2} & -\eta & 0 \\
1 & 0 & 0 & \eta
\end{array}\right)
$$

Note that the perturbation is proportional to the crab cavity strength $\xi_{c}$. Note also that $\eta$ here now refers to the dispersion at the crab cavity while we assume the dispersion at the rf acceleration cavity to vanish.

First we compute the $r_{j k \xi}$ :

$$
\begin{aligned}
r_{11 \xi} & =i \xi_{c} G_{x}, \quad r_{1-1 \xi}=-\xi_{c}\left(\left(i+\alpha_{x}\right) \eta+\beta_{x} \eta^{\prime}\right), \\
r_{12 \xi} & =-i \xi_{c} \frac{\left(i+\alpha_{x}\right)\left(-i+\alpha_{z}\right) \eta^{2}+\beta_{x}\left(\beta_{z}+\left(-i+\alpha_{z}\right) \eta \eta^{\prime}\right)}{2 \sqrt{\beta_{x} \bar{\beta}_{z}}} \\
r_{1-2 \xi} & =\xi_{c} \frac{\left(i+\alpha_{x}\right)\left(i+\alpha_{z}\right) \eta^{2}+\beta_{x}\left(\beta_{z}+\left(i+\alpha_{z}\right) \eta \eta^{\prime}\right)}{2 \sqrt{\beta_{x} \beta_{z}}} \\
r_{22 \xi} & =i \xi_{c} \eta \alpha_{z}, \quad r_{2-2 \xi}=\xi_{c}\left(-i+\alpha_{z}\right) \eta .
\end{aligned}
$$

The rest of the $r_{j k}$ can be gotten using (130) and (131).

Next, we construct $\xi, \Delta \mu, \phi$ and $\bar{\mu}$ for each of the resonances and from these compute $\theta, \mu_{1}$, and $\mu_{2}$.

\section{Sum/difference resonance}

In this case, examining the perturbation matrix (226) we find that the determinant of the off-diagonal submatrices are not zero. We thus expect a difference between the coupling parameters for the sum and difference resonances. Indeed, we find

$$
\xi_{ \pm}=\xi_{c} \sqrt{\frac{a \overline{\beta_{x}}}{\mu_{s}} \mp 2 \eta^{2}}
$$

Note that $\xi_{-}^{2}-\xi_{+}^{2}=4 \xi_{c}^{2} \eta^{2}$ which is consistent with Eq. (C5) from Appendix C. We see that in the case that the dispersion at the crab cavity is zero, the coupling parameter is inversely proportional to the square root of the synchrotron tune. Thus, we expect the sum and difference resonances to get stronger for small synchrotron tune. The splitting parameter is given by

$$
\Delta \mu=\mu_{x}+\mu_{z}+\xi_{c}\left(G_{x}+\eta \alpha_{z}\right)
$$

for the sum resonance, and

$$
\Delta \mu=\mu_{x}-\mu_{z}+\xi_{c}\left(G_{x}-\eta \alpha_{z}\right)
$$

for the difference resonance. $\mathcal{G}_{x}$ was defined in Eq. (218). 
The phase $\phi$ is given by

$$
\phi=\arg \left[\frac{a \beta_{x}}{\mu_{s}}-\eta^{2}+i \eta G_{x}\right]
$$

for the sum resonance, and

$$
\phi=\arg \left[-\eta G_{x}-i\left(\frac{a \beta}{\mu_{s}}-\eta^{2}\right)\right]
$$

for the difference resonance. For the case of no dispersion at the crab cavity, the difference resonance has $\phi=-\frac{\pi}{2}$ and the sum resonance has $\phi=0$. The average shift in the phase advance, $\bar{\mu}$ is given by

$$
\bar{\mu}=\xi_{c}\left(G_{x} \pm \eta \alpha_{z}\right)
$$

with the plus sign for the difference resonance and the minus sign for the sum resonance.

For the sum resonance, we find an instability for

$$
\xi_{c} \sqrt{\frac{a \beta_{x}}{\mu_{s}}-2 \eta^{2}}>\mu_{x}+\mu_{z}+\xi_{c}\left(G_{x}+\eta \alpha_{z}\right) .
$$

\section{Integer/half-integer resonance}

For the $x$ integer resonance the coupling parameter is

$$
\xi^{2}=4 \beta_{x} \mathcal{H}_{x} \xi_{c}^{2}+\frac{4 a \beta_{x} G_{x}}{\mu_{s}^{2}} \xi_{c}^{3}+\frac{a^{2} \beta_{x}^{2}}{\mu_{s}^{4}} \xi_{c}^{4} .
$$

The splitting parameter is

$$
\Delta \mu=2 \mu_{x}+2 \xi_{c} \mathcal{G}+\xi_{c}^{2} \frac{a \beta_{x}}{\mu_{s}^{2}}
$$

and the phase, to lowest order in $\mu_{z}$ and $\xi_{c}$ is

$$
\phi=\pi \text {. }
$$

Out of $\xi$ and $\Delta \mu$, we construct

$$
\begin{aligned}
\mu_{1}^{2}= & \left(\mu_{x}+\xi_{c} \mathcal{G}_{x}\right)^{2}+\left(\mu_{x}+\xi_{c} \mathcal{G}_{x}\right) \frac{a \beta_{x}}{\mu_{s}^{2}} \xi_{c}^{2} \\
& -\frac{a \beta_{x} G_{x}}{\mu_{s}^{2}} \xi_{c}^{3}-\beta_{x} \mathcal{H}_{x} \xi_{c}^{2} .
\end{aligned}
$$

From this, we can see that for $\mu_{x}<0$ (or close to $2 \pi$ ) and for the case of no dispersion, we can have an instability if

$$
\frac{a \beta_{x}}{\mu_{s}^{2}} \xi_{c}^{2}>\left|\mu_{x}\right| \quad\left(\mu_{x}<0\right) .
$$

At larger $\mu_{z}$ and with nonnegligable dispersion at the crab cavity and for the case $G_{x}=0$, we find an instability for

$$
\eta \xi_{c}>\left|\mu_{x}\right|
$$

for both positive and negative $\mu_{x}$.

For the half-integer resonance in $x$, we find a coupling parameter

$$
\xi^{2}=4 \beta_{x} \mathcal{H}_{x} \xi_{c}^{2}-a \beta_{x} G_{x} \xi_{c}^{3}+\frac{1}{16} a^{2} \beta_{x}^{2} \xi_{c}^{4} .
$$

The splitting parameter is

$$
\Delta \mu=2\left(\mu_{x}-\pi\right)+2 \xi_{c} G_{x}-\frac{1}{4} a \beta_{x} \xi_{c}^{2}
$$

and the phase is

$$
\phi=\arg \left(\frac{1}{8} a \beta_{x} \xi_{c}^{2}-G_{x} \xi_{c}-i \eta\right)
$$

which vanishes when there is no dispersion at the crab cavity. Computing $\Delta \mu^{2}-\xi^{2}$ and noting the cancellation of terms, we find

$$
\begin{aligned}
\left(\mu_{1}-\pi\right)^{2}= & {\left[\left(\mu_{x}-\pi\right)+\xi_{c} \mathcal{G}_{x}\right]^{2}-\left[\left(\mu_{x}-\pi\right)\right.} \\
& \left.+2 \xi_{c} \mathcal{G}_{x}\right] \frac{a \beta_{x}}{4} \xi_{c}^{2}-\beta_{x} \mathcal{H}_{x} \xi_{c}^{2} \\
& +\frac{1}{4} a \beta_{x} \mathcal{G}_{x} \xi_{c}^{3} .
\end{aligned}
$$

For the case of $G_{x}=0$, we can have an instability for

$$
\left(\mu_{x}-\pi\right) \frac{a \beta_{x}}{4} \xi_{c}^{2}+\eta^{2} \xi_{c}^{2}>\left(\mu_{x}-\pi\right)^{2} .
$$

Next for the integer resonance in $z$, we find

$$
\begin{aligned}
& \xi^{2}=4 \eta^{2} \xi_{c}^{2}+\frac{a^{2} \beta_{x}^{2} \cot ^{2}\left(\frac{\mu_{x}}{2}\right)}{4 \mu_{s}^{2}} \xi_{c}^{4}, \\
& \Delta \mu=2 \mu_{z}-\frac{1}{2} \frac{a \beta_{x}}{\mu_{s}} \cot \left(\frac{\mu_{x}}{2}\right) \xi_{c}^{2},
\end{aligned}
$$

and

$$
\phi=\arg \left[\frac{a \beta_{x} \cot \left(\frac{\mu_{x}}{2}\right)}{4 \mu_{s}} \xi_{c}^{2}-i \eta \xi_{c}\right] .
$$

For the case where $\eta=0$, the phase $\phi$ is 0 for $\mu_{x}>\pi$ and $\pi$ for $\mu_{x}<\pi$. Computing $\Delta \mu^{2}-\xi^{2}$, we find

$$
\mu_{2}^{2}=\mu_{s}^{2}+\frac{a \beta_{x}}{4} \cot \left(\frac{\mu_{x}}{2}\right) \xi_{c}^{2}-\eta^{2} \xi_{c}^{2} .
$$

From this, we can see that there is an instability if

$$
\frac{a \beta_{x}}{4} \cot \left(\frac{\mu_{x}}{2}\right) \xi_{c}^{2}-\eta^{2} \xi_{c}^{2}<\mu_{s}^{2} .
$$

When $\eta=0$, this requires $\mu_{x}>\pi$, i.e., the horizontal betatron tune is above a half-integer. Increasing $\eta$ moves this instability to values of $\mu_{x}$ less than $\pi$.

Because in practice the synchrotron phase will not be near $\pi$, we do not consider the $\nu_{z}$ half-integer resonance.

We summarize the results for $\xi, \Delta \mu$, and $\phi$ for each of the resonances in Table III.

\section{PERTURBATIVE EXPRESSIONS FOR GLOBAL DAMPING AND DIFFUSION}

We have given perturbative expressions for the eigenvectors of the one-turn map $M$ near all of the linear resonances. As discussed at the end of Sec. IIC, we use these eigenvectors to find the local damping and diffusion coefficients which can then be integrated to give the global values which determine the equilibrium invariants. In this 
TABLE II. Resonances for dispersive rf cavity.

\begin{tabular}{lccccc}
\hline \hline Reso. & Condition & $\Delta \mu(\bmod 2 \pi)$ & $\xi$ & $\phi$ & $\bar{\mu}$ \\
\hline Sum & $\mu_{x}+\mu_{z}=2 \pi n$ & $\mu_{x}+\mu_{z}+\frac{r}{a} \mathcal{H}_{x}$ & $\sqrt{\frac{\mathcal{H}_{x}}{a}\left|\mu_{z}\right|^{\frac{3}{2}}}$ & $\arg \left(-\left(i+\alpha_{x}\right) \eta+\beta_{x} \eta^{\prime}\right)$ & $-\mu_{z}^{2} \frac{\mathcal{H}_{x}}{2 a}$ \\
Dif. & $\mu_{x}-\mu_{z}=2 \pi n$ & $\mu_{x}-\mu_{z}+\frac{r}{a} \mathcal{H}_{x}$ & $\sqrt{\frac{\mathcal{H}_{x}}{a}\left|\mu_{z}\right|^{\frac{3}{2}}}$ & $\arg \left(i\left(i+\alpha_{x}\right) \eta+\beta_{x} \eta^{\prime}\right)$ & $-\mu_{z}^{2} \frac{\mathcal{H}_{x}}{2 a}$ \\
Int $(x)$ & $\mu_{x}=2 \pi n$ & $2 \mu_{x}+\mathcal{O}\left(\frac{\mathcal{H}_{x}}{a} \mu_{z}^{4}\right)$ & $\mathcal{O}\left(\frac{\mathcal{H}_{x}}{a} \mu_{z}^{4}\right)$ & $2 \arg \left(G_{x}+i \eta\right)$ & 0 \\
Int $(z)$ & $\mu_{z}=2 \pi n$ & $2 \mu_{z}-\frac{\mu_{z} \mathcal{H}_{x}}{2 a} \cot \left(\frac{\mu_{x}}{2}\right)$ & $\frac{\mu_{s} \mathcal{H}_{x}}{2 a}\left|\cot \left(\frac{\mu_{x}}{2}\right)\right|$ & 0 & 0 \\
$\frac{1}{2}$-int $(x)$ & $\mu_{x}=\pi(2 n+1)$ & $2\left(\mu_{x}-\pi\right)-\frac{\mathcal{H}_{x}}{a} \mu_{z}^{2}$ & $\mu_{z}^{2} \frac{\mathcal{H}_{x}}{a}$ & $2 \arg \left(G_{x}+i \eta\right)$ & 0 \\
\hline \hline
\end{tabular}

TABLE III. Resonances for crab cavity.

\begin{tabular}{lccccc}
\hline \hline Reso. & Condition & $\Delta \mu(\bmod 2 \pi)$ & $\xi^{2}$ & $\phi$ & $\bar{\mu}$ \\
\hline Sum & $\mu_{x}+\mu_{z}=2 \pi n$ & $\mu_{x}+\mu_{z}+\xi_{c}\left(G_{x}+\eta \alpha_{z}\right)$ & $\xi_{c}^{2}\left(\frac{a \beta_{x}}{\mu_{s}}-2 \eta^{2}\right)$ & $\arg \left(\frac{a \beta_{x}}{\mu_{s}}-\eta^{2}+i \eta G_{x}\right)$ & $\xi_{c}\left(G_{x}-\eta \alpha_{z}\right)$ \\
Diff. & $\mu_{x}-\mu_{z}=2 \pi n$ & $\mu_{x}-\mu_{z}+\xi_{c}\left(G_{x}-\eta \alpha_{z}\right)$ & $\xi_{c}^{2}\left(\frac{\beta_{x}}{\mu_{s}}+2 \eta^{2}\right)$ & $\arg \left(-\eta G_{x}-i\left(\frac{a \beta}{\mu_{s}}-\eta^{2}\right)\right)$ & $\xi_{c}\left(G_{x}+\eta \alpha_{z}\right)$ \\
Int $(x)$ & $\mu_{x}=2 \pi n$ & $2 \mu_{x}+2 \xi_{c} \mathcal{G}_{x}+\xi_{c}^{2} \frac{a \beta_{x}}{\mu_{s}^{s}}$ & $4 \beta_{x} \mathcal{H} \mathcal{H}_{x} \xi_{c}^{2}+\frac{4 a \beta_{x} G_{x}}{\mu_{s}} \xi_{c}^{3}+\frac{a^{2} \beta_{x}^{2}}{\mu_{s}^{2}} \xi_{c}^{4}$ & $\pi$ & 0 \\
Int $(z)$ & $\mu_{z}=2 \pi n$ & $2 \mu_{z}-\frac{1}{2} \frac{a \beta_{x}}{\mu_{s}} \cot \left(\frac{\mu_{x}}{2}\right) \xi_{c}^{2}$ & $4 \eta^{2} \xi_{c}^{2}+\frac{a^{2} \beta_{x}^{2} \cot \left(\frac{\mu_{x}}{4}\right)}{4 \mu_{s}^{2}} \xi_{c}^{4}$ & $\arg \left(\frac{a \beta_{x} \cot \left(\frac{\mu_{x}}{2}\right)}{4 \mu_{s}} \xi_{c}^{2}-i \eta \xi_{c}\right)$ & 0 \\
$\frac{1}{2}$-int $(x)$ & $\mu_{x}=\pi(2 n+1)$ & $2\left(\mu_{x}-\pi\right)+2 \xi_{c} G_{x}-\frac{1}{4} a \beta_{x} \xi_{c}^{2}$ & $4 \beta_{x} \mathcal{H}_{x} \xi_{c}^{2}-a \beta_{x} G_{x} \xi_{c}^{3}+\frac{1}{16} a^{2} \beta_{x}^{2} \xi_{c}^{4}$ & $\arg \left(\frac{1}{8} a \beta_{x} \xi_{c}^{2}-G_{x} \xi_{c}-i \eta\right)$ & 0 \\
\hline \hline
\end{tabular}

section we apply our perturbative expressions for the eigenvectors to find perturbative expressions for the invariants, damping and diffusion coefficients, and ultimately equilibrium emittances and beam second moments. We work in betatron coordinates, so for the local damping and diffusion matrices, we use $D_{\beta}$ and $B_{\beta}$, which were defined in Eqs. (69) and (79).

\section{A. Invariants}

With the addition of coupling near a linear resonance, the invariants $G_{x}$ and $G_{z}$ become perturbed to $G_{1}$ and $G_{2}$. We compute these approximately by using the lowest order perturbed eigenvectors $\tilde{\boldsymbol{v}}_{j 0}$ derived in Sec. III.

\section{Sum/difference resonance}

First consider the sum [Eqs. (151), (152), (169), and (170)] and difference resonances [Eqs. (149), (150), (177), and (178)]. We find

$$
\begin{aligned}
G_{1} & =\cosh ^{2}\left(\frac{\theta}{2}\right) G_{x}+\sinh ^{2}\left(\frac{\theta}{2}\right) G_{z}+\sinh (\theta) G_{c}^{+}, \\
G_{2} & =\sinh ^{2}\left(\frac{\theta}{2}\right) G_{x}+\cosh ^{2}\left(\frac{\theta}{2}\right) G_{z}+\sinh (\theta) G_{c}^{+},
\end{aligned}
$$

for the sum resonance, and

$$
\begin{aligned}
& G_{1}=\cos ^{2}\left(\frac{\theta}{2}\right) G_{x}+\sin ^{2}\left(\frac{\theta}{2}\right) G_{z}-\sin (\theta) G_{c}^{-}, \\
& G_{2}=\sin ^{2}\left(\frac{\theta}{2}\right) G_{x}+\cos ^{2}\left(\frac{\theta}{2}\right) G_{z}+\sin (\theta) G_{c}^{-},
\end{aligned}
$$

for the difference resonance. We can easily see that the local invariant sum rules (60) are satisfied. Note that in addition to mixing the uncoupled invariants $G_{x}$ and $G_{z}$, an additional term, $G_{c}^{ \pm}$is picked up. This middle term can be related to the additional invariants that exist exactly on resonance (see [10]), which are mixed together by the phase $\phi$. These terms are given by

$$
\begin{aligned}
G_{c}^{+}= & -\frac{1}{2} J\left(e^{i \phi} \boldsymbol{v}_{x}^{*} \boldsymbol{v}_{-z}^{T}+e^{-i \phi} \boldsymbol{v}_{x} \boldsymbol{v}_{-z}^{\dagger}+e^{-i \phi} \boldsymbol{v}_{-z}^{*} \boldsymbol{v}_{x}^{T}\right. \\
& \left.+e^{i \phi} \boldsymbol{v}_{-z} \boldsymbol{v}_{x}^{\dagger}\right) J, \\
G_{c}^{-}= & -\frac{1}{2} J\left(e^{i \phi} \boldsymbol{v}_{x}^{*} \boldsymbol{v}_{z}^{T}+e^{-i \phi} \boldsymbol{v}_{x} \boldsymbol{v}_{z}^{\dagger}+e^{-i \phi} \boldsymbol{v}_{z}^{*} \boldsymbol{v}_{x}^{T}\right. \\
+ & \left.e^{i \phi} \boldsymbol{v}_{z} \boldsymbol{v}_{x}^{\dagger}\right) J .
\end{aligned}
$$

Writing them out explicitly, we find

$$
G_{c}^{ \pm}=\left(\begin{array}{cc}
0_{2 \times 2} & \bar{G}_{c}^{ \pm} \\
\bar{G}_{c}^{ \pm T} & 0_{2 \times 2}
\end{array}\right),
$$

with these submatrices given by

$$
\bar{G}_{c}^{+}=\frac{1}{2}\left(\begin{array}{cc}
\frac{\left(-1+\alpha_{x} \alpha_{z}\right) \cos \phi+\left(\alpha_{x}+\alpha_{z}\right) \sin \phi}{\sqrt{\beta_{x} \beta_{z}}} & \sqrt{\beta_{z} / \beta_{x}}\left(\alpha_{x} \cos \phi+\sin \phi\right) \\
\sqrt{\beta_{x} / \beta_{z}}\left(\alpha_{z} \cos \phi+\sin \phi\right) & \sqrt{\beta_{x} \beta_{z}} \cos \phi
\end{array}\right),
$$

and 


$$
\bar{G}_{c}^{-}=\frac{1}{2}\left(\begin{array}{cc}
-\frac{\left(\alpha_{x}-\alpha_{z}\right) \cos \phi+\left(1+\alpha_{x} \alpha_{z}\right) \sin \phi}{\sqrt{\beta_{x} \beta_{z}}} & \sqrt{\beta_{z} / \beta_{x}}\left(\cos \phi-\alpha_{x} \sin \phi\right) \\
-\sqrt{\beta_{x} / \beta_{z}}\left(\cos \phi+\alpha_{z} \sin \phi\right) & -\sqrt{\beta_{x} \beta_{z}} \sin \phi
\end{array}\right)
$$

for the sum and difference resonances, respectively. Note that these additional coupling terms all contain a factor of $\cos \phi$ or $\sin \phi$. In the typical case, this term will oscillate many times around the ring. When used to construct the global diffusion coefficient, this means that this term will not contribute substantially. However, there are exceptions to this statement, and further, they are required for computing the detailed local moment matrix $\Sigma$.

\section{Integer/half-integer resonance}

Now consider the integer and half-integer resonances. The eigenvector is of the form (151). Consider the $x$ integer/half-integer resonances. Then $\boldsymbol{v}_{j 0}=\boldsymbol{v}_{x}$ and $\boldsymbol{v}_{k 0}=$ $v_{-x}$. Constructing the invariant out of the eigenvector gives

$$
G_{1}=\cosh \theta G_{x}+\sinh \theta G_{c},
$$

where here

$$
G_{c}=\left(\begin{array}{cccc}
\frac{\left(-1+\alpha_{x}^{2}\right) \cos \phi+2 \alpha_{x} \sin \phi}{\beta_{x}} & \alpha_{x} \cos \phi+\sin \phi & 0 & 0 \\
\alpha_{x} \cos \phi+\sin \phi & \beta_{x} \cos \phi & 0 & 0 \\
0 & 0 & 0 & 0 \\
0 & 0 & 0 & 0
\end{array}\right) .
$$

In these expressions, the coupling angle $\theta$ and phase $\phi$ are the appropriate expression for the integer or half-integer resonance as the case may be. Note that we could also obtain the additional coupling term $G_{c}$ by setting $\beta_{z}=\beta_{x}$ and $\alpha_{z}=\alpha_{x}$ in Eq. (255). Note that we only have two terms in these expressions for the invariant, versus three for the sum and difference resonances. This is because the invariants corresponding to $v_{x}$ and $v_{-x}$ are identical. For $z$ we just replace all the $x$ 's with $z$ 's.

Note that for an integer or half-integer resonance, the invariant remains nonzero only in the $x$ or $z$ on-diagonal block to the order we compute here. One could thus interpret the new invariant as defining new $\beta, \alpha$, and $\gamma$ rather than as a perturbed invariant. This allows us to compare our results to Courant and Snyder [4]. If we do this, we compare Eqs. (257) and (258) to Eq. (99) to derive a perturbed beta function

$$
\beta_{1}=\beta_{x}(\cosh \theta+\cos \phi \sinh \theta),
$$

which reduces to $\beta_{x}$ when the coupling is turned off. The value of $\phi$ varies around the ring via Eq. (197) from its initial value as given in Table I. This is a well-known effect referred to as a beta beat, the periodic oscillation of the beta function resulting from a perturbation. Note that the initial value will be different for the integer and half-integer resonances. Also note that because $\cosh \theta>|\sinh \theta|, \beta_{1}$ will always be positive, although the range of the oscillations becomes large as the instability $(\theta \rightarrow \infty)$ is approached.

\section{B. Diffusion coefficients}

From the invariants, we can find the local diffusion coefficients by right-multiplying by the diffusion matrix $D_{\beta}$ and taking the trace.

\section{Sum/difference resonance}

The results for the sum/difference resonance are

$d_{1}^{+}=\cosh ^{2}\left(\frac{\theta}{2}\right) d_{x}+\sinh ^{2}\left(\frac{\theta}{2}\right) d_{z}+\sinh (\theta) d_{c}^{+}$,

$d_{2}^{+}=\sinh ^{2}\left(\frac{\theta}{2}\right) d_{x}+\cosh ^{2}\left(\frac{\theta}{2}\right) d_{z}+\sinh (\theta) d_{c}^{+}$,

$d_{1}^{-}=\cos ^{2}\left(\frac{\theta}{2}\right) d_{x}+\sin ^{2}\left(\frac{\theta}{2}\right) d_{z}+\sin (\theta) d_{c}^{-}$,

$d_{2}^{-}=\sin ^{2}\left(\frac{\theta}{2}\right) d_{x}+\cos ^{2}\left(\frac{\theta}{2}\right) d_{z}-\sin (\theta) d_{c}^{-}$,

with

$$
d_{c}^{ \pm}=\operatorname{Tr}\left[G_{c}^{ \pm} D_{\beta}\right] .
$$

To get the global diffusion coefficient $\bar{d}_{a}$, we must integrate the local diffusion coefficients $d_{a}$ around the ring. For $d_{x}$ and $d_{z}$ this integration simply reproduces the global uncoupled diffusion coefficients $\bar{d}_{x}$ and $\bar{d}_{z}$. The extra term, $d_{c}$, which comes from the extra term in the invariants $G_{c}$, however, represents a new set of lattice functions to be integrated. It would be a substantial complication if this full integration were required since it would require detailed knowledge of the lattice. Note however, that as with $G_{c}$, all of the terms in $d_{c}$ are proportional to $\cos \phi$ or $\sin \phi$. As we transform the $d_{a}$ around the ring, in addition to the lattice functions $\beta_{x, z}$ and $\eta_{x, z}$ evolving, the phase $\phi$ will also evolve as was discussed in Sec. III E.

The net change in $\phi$ around the ring is $\mu_{x}-\mu_{z}$ for the sum resonance and $\mu_{x}+\mu_{z}$ for the difference resonance. We can write this is as

$$
\Delta \phi=2 \pi n+\left[\mu_{x} \mp \mu_{z}\right],
$$

where the square brackets represent taking a modulus of $2 \pi$. Near resonance this term is small. For synchrobetaron coupling, typically $n$ is somewhat large, since $\nu_{z}$ is close to zero and $\nu_{x}$ is typically much greater than 1 . The result of this argument is that the middle term in the local diffusion coefficient $d_{c}$ will integrate to a small value in the typical 
situation, especially so if we assume some symmetry in the lattice. ${ }^{14}$ Thus, for our purposes here, we ignore this extra term in our global expressions, ${ }^{15}$

$$
\begin{aligned}
& \bar{d}_{1}^{+}=\cosh ^{2}\left(\frac{\theta}{2}\right) \bar{d}_{x}+\sinh ^{2}\left(\frac{\theta}{2}\right) \bar{d}_{z}, \\
& \bar{d}_{2}^{+}=\sinh ^{2}\left(\frac{\theta}{2}\right) \bar{d}_{x}+\cosh ^{2}\left(\frac{\theta}{2}\right) \bar{d}_{z}, \\
& \bar{d}_{1}^{-}=\cos ^{2}\left(\frac{\theta}{2}\right) \bar{d}_{x}+\sin ^{2}\left(\frac{\theta}{2}\right) \bar{d}_{z} \\
& \bar{d}_{2}^{-}=\sin ^{2}\left(\frac{\theta}{2}\right) \bar{d}_{x}+\cos ^{2}\left(\frac{\theta}{2}\right) \bar{d}_{z}
\end{aligned}
$$

\section{Integer/half-integer resonance}

We now consider the diffusion coefficients for the integer and half-integer resonances. For these resonances, we find

$$
d_{2}^{n}=d_{z} \cosh \theta+d_{c} \sinh \theta,
$$

where

$$
d_{c}=\operatorname{Tr}\left(G_{c}^{n z} D_{z z}\right),
$$

with the results for $x$ given by replacing $z$ with $x$ in these expressions.

We must now integrate the local diffusion coefficient around the ring to get the global diffusion coefficient. For the case of $x$, applying the same argument as in the sum and difference resonances, we find that the extra term $d_{c}$ integrates to a small value and we disregard it. For the longitudinal direction, however, there is no change in $\phi$ around the ring, and thus we must keep $d_{c}$, with its initial value. Thus, for the global diffusion coefficients, we take

$$
\bar{d}_{1}=\cosh (\theta) \bar{d}_{x} .
$$

It may also be useful to apply Eqs. (162) or (163) for the integer or half-integer resonances to express in terms of $\mu_{1}$ and $\Delta \mu$. This is for near the integer or half-integer $x$ resonance and

$$
\bar{d}_{2}=(\cosh \theta+\cos \phi \sinh \theta) \bar{d}_{z} .
$$

near the integer $z$ resonance. For the $x$ resonances, the $\bar{d}_{2}$ is not strongly effected, while for the $z$ resonance, $\bar{d}_{1}$ is not

\footnotetext{
${ }^{14}$ In the case of $x-y$ betatron coupling in which $\nu_{x} \approx \nu_{y}$ is possible, this argument will need to be revised.

${ }^{15}$ We note here that in case one wanted to compute these middle terms exactly, then, $\cos \phi$ and $\sin \phi$ can be expanded in terms of $\cos \phi\left(s_{1}\right), \sin \phi\left(s_{1}\right)$ and cosine and sine of the phase advances $\psi_{x 12}$ and $\psi_{z 12}$. We could then write out the expressions $b_{c}$ and $d_{c}$ explicitly in terms of unperturbed lattice functions. Then, for a given lattice, one could compute these integrals once and for all, and not have to recompute different integrals, depending on the form of the coupling, one would simply take different linear combinations of these integrals depending on the value of $\phi\left(s_{1}\right)$.
}

strongly effected and we assume there is no change in these quantities.

\section{Local damping coefficients}

Next we find the local damping coefficients. We do this by constructing the matrix $A=U^{-1} B_{\beta} U\left[B_{\beta}\right.$ is defined in Eq. (79)] with $U$ constructed from the perturbed eigenvectors for each resonance. From $A$ we find $b_{a}=A_{a a}+$ $A_{-a-a}$.

\section{Sum/difference resonance}

The damping coefficients for the sum and difference resonances are given by

$$
\begin{aligned}
& b_{1}^{+}=b_{x \beta} \cosh ^{2}\left(\frac{\theta}{2}\right)-b_{z \beta} \sinh ^{2}\left(\frac{\theta}{2}\right)+\sinh (\theta) b_{c}^{+}, \\
& b_{2}^{+}=-b_{x \beta} \sinh ^{2}\left(\frac{\theta}{2}\right)+b_{z \beta} \cosh ^{2}\left(\frac{\theta}{2}\right)-\sinh (\theta) b_{c}^{+}, \\
& b_{1}^{-}=b_{x \beta} \cos ^{2}\left(\frac{\theta}{2}\right)+b_{z \beta} \sin ^{2}\left(\frac{\theta}{2}\right)+\sin (\theta) b_{c}^{-}, \\
& b_{2}^{-}=b_{x \beta} \sin ^{2}\left(\frac{\theta}{2}\right)+b_{z \beta} \cos ^{2}\left(\frac{\theta}{2}\right)-\sin (\theta) b_{c}^{-},
\end{aligned}
$$

with

$$
\begin{aligned}
& b_{x \beta}=\operatorname{Tr}\left[B_{x x}\right]=b_{x}-\eta b_{\delta x}, \\
& b_{z \beta}=\operatorname{Tr}\left[B_{z z}\right]=b_{z}+\eta b_{\delta x},
\end{aligned}
$$

where

$$
\begin{aligned}
b_{c}^{+}= & \frac{1}{2 \sqrt{\beta_{x} \beta_{z}}}\left[\left(-\left(b_{x}-b_{z}+b_{\delta x} \eta\right)\left(\left(\alpha_{x}+\alpha_{z}\right) \eta\right.\right.\right. \\
& \left.\left.+\beta_{x} \eta^{\prime}\right)\right) \cos \phi+\left(( b _ { x } - b _ { z } ) \left(-\eta+\alpha_{x} \alpha_{z} \eta\right.\right. \\
& \left.+\alpha_{z} \beta_{x} \eta^{\prime}\right)+b_{\delta x}\left(\beta_{x} \beta_{z}+\left(1-\alpha_{x} \alpha_{z}\right) \eta^{2}\right. \\
& \left.\left.\left.-\alpha_{z} \beta_{x} \eta \eta^{\prime}\right)\right) \sin \phi\right], \\
b_{c}^{-}= & \frac{1}{2 \sqrt{\beta_{x} \beta_{z}}}\left[\left(b_{x}-b_{z}\right)\left(\left(1+\alpha_{x} \alpha_{z}\right) \eta+\alpha_{z} \beta_{x} \eta^{\prime}\right)\right. \\
& -b_{\delta x}\left(-\beta_{x} \beta_{z}+\eta^{2}+\alpha_{x} \alpha_{z} \eta^{2}+\alpha_{z} \beta_{x} \eta \eta^{\prime}\right) \cos \phi \\
& -\left(b_{x}-b_{z}-b_{\delta x} \eta\left[\left(\left(\alpha_{x}-\alpha_{z}\right) \eta+\beta_{x} \eta^{\prime}\right] \sin \phi\right] .\right.
\end{aligned}
$$

Again, as with the local diffusion coefficients, we find an extra term in $b_{a}$, in addition to the uncoupled damping coefficients $b_{x \beta}$ and $b_{z \beta}$. Using the same argument as for the diffusion coefficients, we find that this quantity integrates to a small value and we will discard it in our global expressions. In addition, note that $b_{c}^{ \pm}$is proportional to either $1 / \beta_{z}$ or $b_{\delta x}$. For the case of synchrobetatron coupling, both $1 / \beta_{z}$ and $b_{\delta x}$ are small, and so this term is intrinsically small. The integration of $b_{x \beta}$ and $b_{z \beta}$ give the 
global uncoupled damping decrements, $\chi_{x}$ and $\chi_{z}$. Thus, for our global coupled damping coefficients, we take

$$
\begin{aligned}
& \chi_{1}^{+}=\chi_{x} \cosh ^{2}\left(\frac{\theta}{2}\right)-\chi_{z} \sinh ^{2}\left(\frac{\theta}{2}\right), \\
& \chi_{2}^{+}=-\chi_{x} \sinh ^{2}\left(\frac{\theta}{2}\right)+\chi_{z} \cosh ^{2}\left(\frac{\theta}{2}\right) \\
& \chi_{1}^{-}=\chi_{x} \cos ^{2}\left(\frac{\theta}{2}\right)+\chi_{z} \sin ^{2}\left(\frac{\theta}{2}\right) \\
& \chi_{2}^{-}=\chi_{x} \sin ^{2}\left(\frac{\theta}{2}\right)+\chi_{z} \cos ^{2}\left(\frac{\theta}{2}\right)
\end{aligned}
$$

The damping decrements for the sum resonance show an interesting effect. One of $\chi_{1,2}$ will become negative for a finite value of $\theta$. Specifically, suppose that $\chi_{z}>\chi_{x}$ which is typically the case. Then $\chi_{1}$ vanishes when

$$
\sqrt{\frac{\chi_{z}}{\chi_{x}}}=\operatorname{coth}\left(\frac{\theta}{2}\right)
$$

For $\theta$ larger than this, $\chi_{1}$ becomes negative, and there is an instability. This is analogous to the case where the damping partition number $\mathcal{D}$ is greater than 1 , in which case, we can see from Eq. (108) that $\chi_{x}$ is likewise negative, indicating an instability. We refer to this as an "antidamping instability" in this paper.

\section{Integer/half-integer resonances}

For the integer/half-integer resonance, we find that the local damping coefficients are unchanged. This is clear because the perturbation only changes the $U$ matrix in either the upper left or lower right $2 \times 2$ block, depending on which resonance being considered. But this reduces the problem to the $1 \mathrm{D}$ case. Consider the $\nu_{z}$ integer or halfinteger resonance. The damping coefficient for mode 2 is given by

$$
b_{2}=\operatorname{Tr}\left(U_{2}^{-1} B_{z z} U_{2}\right)=\operatorname{Tr}\left(B_{z z}\right)=b_{z \beta},
$$

where $U_{2}$ is the submatrix of $U$ built out of $\tilde{\boldsymbol{v}}_{20}$ and $\tilde{\boldsymbol{v}}_{-20}$. We have used the cyclic property of the trace. In other words, the damping decrements are unchanged to lowest order near the integer/half-integer resonances.

\section{Equilibrium eigenemittances}

We summarize the results for the diffusion and damping in Tables IV and V. The reader can verify that all the quantities satisfy the sum rules discussed in Sec. II D.

From the global quantities $\bar{d}_{1,2}$ and $\chi_{1,2}$, we find the equilibrium average values of the invariants from Eq. (47). We quote RMS eigenemittances, which are $1 / 2$ the value of the average eigeninvariants.

For the sum resonance, we find

$$
\epsilon_{1}^{+}=\frac{\cosh ^{2} \frac{\theta}{2} \bar{d}_{x}+\sinh ^{2} \frac{\theta}{2} \bar{d}_{z}}{4\left(\cosh ^{2} \frac{\theta}{2} \chi_{x}-\sinh ^{2} \frac{\theta}{2} \chi_{z}\right)},
$$

$$
\epsilon_{2}^{+}=\frac{\sinh ^{2} \frac{\theta}{2} \bar{d}_{x}+\cosh ^{2} \frac{\theta}{2} \bar{d}_{z}}{4\left(-\sinh ^{2} \frac{\theta}{2} \chi_{x}+\cosh ^{2} \frac{\theta}{2} \chi_{z}\right)},
$$

while for the difference resonance, we find

$$
\begin{aligned}
& \epsilon_{1}^{-}=\frac{\cos ^{2} \frac{\theta}{2} \bar{d}_{x}+\sin ^{2} \frac{\theta}{2} \bar{d}_{z}}{4\left(\cos ^{2} \frac{\theta}{2} \chi_{x}+\sin ^{2} \frac{\theta}{2} \chi_{z}\right)}, \\
& \epsilon_{2}^{-}=\frac{\sin ^{2} \frac{\theta}{2} \bar{d}_{x}+\cos ^{2} \frac{\theta}{2} \bar{d}_{z}}{4\left(\sin ^{2} \frac{\theta}{2} \chi_{x}+\cos ^{2} \frac{\theta}{2} \chi_{z}\right)} .
\end{aligned}
$$

Note that in the case where $\chi_{x}=\chi_{z}$, we find that

$$
\begin{aligned}
& \epsilon_{1}^{+}=\cosh ^{2} \frac{\theta}{2} \epsilon_{x}+\sinh ^{2} \frac{\theta}{2} \epsilon_{z}, \\
& \epsilon_{2}^{+}=\sinh ^{2} \frac{\theta}{2} \epsilon_{x}+\cosh ^{2} \frac{\theta}{2} \epsilon_{z},
\end{aligned}
$$

for the sum resonance and

$$
\begin{aligned}
& \epsilon_{1}^{-}=\cos ^{2} \frac{\theta}{2} \epsilon_{x}+\sin ^{2} \frac{\theta}{2} \epsilon_{z} \\
& \epsilon_{2}^{-}=\sin ^{2} \frac{\theta}{2} \epsilon_{x}+\cos ^{2} \frac{\theta}{2} \epsilon_{z}
\end{aligned}
$$

for the difference resonance. Thus, in this case, it makes sense to talk about emittance coupling: the effect of the coupling is simply to mix together the equilibrium emittances. If we were talking about transverse $x-y$ coupling, $\chi_{x}=\chi_{z}$ would indeed be approximately correct in many situations and this gives a justification for using that concept for betatron coupling. For the case here of synchrobetatron coupling, typically $\chi_{x} \approx \chi_{z} / 2$, and thus the concept of emittance coupling is not precise.

For the integer and half-integer resonances, we saw in Eq. (273) that the damping partition number is not affected by the coupling to lowest order. The global diffusion coefficient is affected. In fact it diverges at the resonance. For the integer and half-integer $x$ resonances, we find an emittance growth of

$$
\frac{\epsilon_{1}}{\epsilon_{x}}=\frac{\bar{d}_{1}}{\bar{d}_{x}}=\cosh \theta
$$

with $\theta$ the appropriate coupling angle. $\epsilon_{z}$ is not strongly affected, and we take $\epsilon_{2}=\epsilon_{z}$. As noted in Eq. (265), one could also write this as

$$
\frac{\epsilon_{1}}{\epsilon_{x}}=\frac{\Delta \mu}{2 \mu_{1}}
$$

for the integer resonance, or

$$
\frac{\epsilon_{1}}{\epsilon_{x}}=\frac{\Delta \mu}{2\left(\mu_{1}-\pi\right)}
$$

for the half-integer resonance. 
TABLE IV. This table contains the diffusion coefficients near each of the linear resonances. The $d$ column gives the local diffusion coefficient for modes 1 and 2. The $d_{c}$ column gives the formula for the extra term $d_{c}$ contained in the corresponding local diffusion coefficient. Finally, the $\bar{d}$ column contains the global diffusion coefficient which is given by integrating the local quantity. The text discusses the approximations used for these expressions. The angle $\theta$ is given by $\tan ^{-1}(\xi / \Delta \mu)$ for the difference resonance and $\tanh ^{-1}(\xi / \Delta \mu)$ for the other resonances where $\xi$ and $\Delta \mu$ are given in Table I. The phase $\phi$ for the integer $z$ resonance is also given in Table I.

\begin{tabular}{|c|c|c|c|c|}
\hline Resonance & Mode & $d$ & $d_{c}$ & $\bar{d}$ \\
\hline \multirow[t]{2}{*}{$\overline{\text { Sum }}$} & 1 & $d_{x} \cosh ^{2} \frac{\theta}{2}+d_{z} \sinh ^{2} \frac{\theta}{2}+d_{c} \sinh \theta$ & & $\cosh ^{2}\left(\frac{\theta}{2}\right) \bar{d}_{x}+\sinh ^{2}\left(\frac{\theta}{2}\right) \bar{d}_{z}$ \\
\hline & 2 & $d_{x} \sinh ^{2} \frac{\theta}{2}+d_{z} \cosh ^{2} \frac{\theta}{2}+d_{c} \sinh \theta$ & $\operatorname{Tr}\left[G_{c}^{+} D_{\beta}\right]$ & $\sinh ^{2}\left(\frac{\theta}{2}\right) \bar{d}_{x}+\cosh ^{2}\left(\frac{\theta}{2}\right) \bar{d}_{z}$ \\
\hline \multirow[t]{2}{*}{ Difference } & 1 & $d_{x} \cos ^{2} \frac{\theta}{2}+d_{z} \sin ^{2} \frac{\theta}{2}+d_{c} \sin \theta$ & & $\cos ^{2}\left(\frac{\theta}{2}\right) \bar{d}_{x}+\sin ^{2}\left(\frac{\theta}{2}\right) \bar{d}_{z}$ \\
\hline & 2 & $d_{x} \sin ^{2} \frac{\theta}{2}+d_{z} \cos ^{2} \frac{\theta}{2}-d_{c} \sin \theta$ & $\operatorname{Tr}\left[G_{c}^{-} D_{\beta}\right]$ & $\sin ^{2}\left(\frac{\theta}{2}\right) \bar{d}_{x}+\cos ^{2}\left(\frac{\theta}{2}\right) \bar{d}_{z}$ \\
\hline \multirow[t]{2}{*}{ Int $/ \frac{1}{2}$-int $(x)$} & 1 & $d_{x} \cosh \theta+d_{c} \sinh \theta$ & & $\bar{d}_{x} \cosh \theta$ \\
\hline & 2 & $d_{z}$ & $\operatorname{Tr}\left[G_{c}^{n x} D_{\beta}\right]$ & $\bar{d}_{z}$ \\
\hline \multirow[t]{2}{*}{ Int $(z)$} & 1 & $d_{x}$ & & $\bar{d}_{x}$ \\
\hline & 2 & $d_{z} \cosh \theta+d_{c} \sinh \theta$ & $\operatorname{Tr}\left[G_{c}^{n z} D_{\beta}\right]$ & $\bar{d}_{z}(\cosh \theta+\cos \phi \sinh \theta)$ \\
\hline
\end{tabular}

TABLE V. This table contains the damping coefficients near each of the linear resonances. The $b$ column gives the local damping coefficient for modes 1 and 2. The $b_{c}$ column tells where to find the formula for the extra term $b_{c}$ contained in the corresponding local damping coefficient. Finally, the $\chi$ column contains the global damping decrement which is given by integrating the local quantity. The approximations used for these expressions are discussed in the text. The angle $\theta$ is given by $\tan ^{-1}(\xi / \Delta \mu)$ for the difference resonance and $\tanh ^{-1}(\xi / \Delta \mu)$ for the other resonances where $\xi$ and $\Delta \mu$ are given in Table I.

\begin{tabular}{|c|c|c|c|c|}
\hline Resonance & Mode & $b$ & $b_{c}$ & $\chi$ \\
\hline \multirow[t]{2}{*}{ Sum } & 1 & $b_{x \beta} \cosh ^{2} \frac{\theta}{2}-b_{z \beta} \sinh ^{2} \frac{\theta}{2}+b_{c} \sinh \theta$ & & $\chi_{x} \cosh ^{2}\left(\frac{\theta}{2}\right)-\chi_{z} \sinh ^{2}\left(\frac{\theta}{2}\right)$ \\
\hline & 2 & $-b_{x \beta} \sinh ^{2} \frac{\theta}{2}+b_{z \beta} \cosh ^{2} \frac{\theta}{2}-b_{c} \sinh \theta$ & Equation (269) & $-\chi_{x} \sinh ^{2}\left(\frac{\theta}{2}\right)+\chi_{z} \cosh ^{2}\left(\frac{\theta}{2}\right)$ \\
\hline \multirow[t]{2}{*}{ Difference } & 1 & $b_{x \beta} \cos ^{2} \frac{\theta}{2}+b_{z \beta} \sin ^{2} \frac{\theta}{2}+b_{c} \sin \theta$ & & $\chi_{x} \cos ^{2}\left(\frac{\theta}{2}\right)+\chi_{z} \sin ^{2}\left(\frac{\theta}{2}\right)$ \\
\hline & 2 & $b_{x \beta} \sin ^{2} \frac{\theta}{2}+b_{z \beta} \cos ^{2} \frac{\theta}{2}-b_{c} \sin \theta$ & Equation (270) & $\chi_{x} \sin ^{2}\left(\frac{\theta}{2}\right)+\chi_{z} \cos ^{2}\left(\frac{\theta}{2}\right)$ \\
\hline \multirow[t]{2}{*}{ Int $/ \frac{1}{2}$-int } & 1 & $b_{x \beta}$ & & $\chi_{x}$ \\
\hline & 2 & $b_{z \beta}$ & & $\chi_{z}$ \\
\hline
\end{tabular}

Now, because $\mu_{z}$ is typically small, we only have an integer resonance. As discussed, for this case, the $\cos \phi$ term does not average away, and we are left with

$$
\frac{\epsilon_{2}}{\epsilon_{z}}=\cosh \theta+\cos \phi \sinh \theta
$$

$\epsilon_{x}$ is not much affected here, and we take $\epsilon_{1}=\epsilon_{x}$. If there were a half-integer $z$ resonance, or indeed, another integer resonance where $\mu_{z}$ is near an integer other than 0 , the second term in this equation would integrate to a small value.

\section{E. Sigma matrices}

In the previous subsection, we gave the expressions for the equilibrium eigenemittances near each of the resonances. One may also wish to know the projected emittances which are more closely related to the typically measured quantities for a beam. Finally, we give expressions for the second moments of the beam distribution at equilibrium. For the sum and difference resonances, using the invariants and Eq. (32), we can derive

$$
\Sigma^{+}=\left(\begin{array}{cc}
\epsilon_{x, \mathrm{pr}}^{+} \Sigma_{x} & \Sigma_{c}^{+} \\
\Sigma_{c}^{+T} & \epsilon_{z, \mathrm{pr}}^{+} \Sigma_{z}
\end{array}\right)
$$

where

$$
\begin{aligned}
& \epsilon_{x, \mathrm{pr}}^{+}=\frac{1}{2}\left\langle g_{1}\right\rangle_{\mathrm{eq}}^{+} \cosh ^{2}\left(\frac{\theta}{2}\right)+\frac{1}{2}\left\langle g_{2}\right\rangle_{\mathrm{eq}}^{+} \sinh ^{2}\left(\frac{\theta}{2}\right), \\
& \epsilon_{z, \mathrm{pr}}^{+}=\frac{1}{2}\left\langle g_{1}\right\rangle_{\mathrm{eq}}^{+} \sinh ^{2}\left(\frac{\theta}{2}\right)+\frac{1}{2}\left\langle g_{2}\right\rangle_{\mathrm{eq}}^{+} \cosh ^{2}\left(\frac{\theta}{2}\right),
\end{aligned}
$$

and

$$
\Sigma^{-}=\left(\begin{array}{cc}
\epsilon_{x, \mathrm{pr}}^{-} \Sigma_{x} & \Sigma_{c}^{-} \\
\Sigma_{c}^{-T} & \epsilon_{z, \mathrm{pr}}^{-} \Sigma_{z}
\end{array}\right)
$$

where

$$
\begin{aligned}
& \epsilon_{x, \mathrm{pr}}^{-}=\frac{1}{2}\left\langle g_{1}\right\rangle_{\mathrm{eq}}^{-} \cos ^{2}\left(\frac{\theta}{2}\right)+\frac{1}{2}\left\langle g_{2}\right\rangle_{\mathrm{eq}}^{-} \sin ^{2}\left(\frac{\theta}{2}\right), \\
& \epsilon_{z, \mathrm{pr}}^{-}=\frac{1}{2}\left\langle g_{1}\right\rangle_{\mathrm{eq}}^{-} \sin ^{2}\left(\frac{\theta}{2}\right)+\frac{1}{2}\left\langle g_{2}\right\rangle_{\mathrm{eq}}^{-} \cos ^{2}\left(\frac{\theta}{2}\right),
\end{aligned}
$$

with 


$$
\Sigma_{c}^{-}=\frac{1}{4}\left[\left\langle g_{1}\right\rangle_{\mathrm{eq}}-\left\langle g_{2}\right\rangle_{\mathrm{eq}}\right] \sin (\theta)\left(-J G_{c}^{-} J\right),
$$

and

$$
\Sigma_{c}^{+}=\frac{1}{4}\left[\left\langle g_{1}\right\rangle_{\mathrm{eq}}+\left\langle g_{2}\right\rangle_{\mathrm{eq}}\right] \sinh (\theta)\left(-J G_{c}^{+} J\right) .
$$

The uncoupled $2 \times 2$ matrices $\Sigma_{x, z}$ are given by

$$
\Sigma_{x, z}=\left(\begin{array}{cc}
\beta_{x, z} & \alpha_{x, z} \\
\alpha_{x, z} & \gamma_{x, z}
\end{array}\right)
$$

$\boldsymbol{\epsilon}_{x(, z), \mathrm{pr}}$ are the projected horizontal and longitudinal emittances. From them, one can, for example, determine the transverse beam size and momenta,

$$
\begin{aligned}
& \left\langle x_{\beta}^{2}\right\rangle=\epsilon_{x, \mathrm{pr}} \beta_{x}, \\
& \left\langle x_{\beta}^{\prime 2}\right\rangle=\epsilon_{x, \mathrm{pr}} \gamma_{x},
\end{aligned}
$$

and likewise for the bunch length and energy spread. Note however that these are in betatron coordinates and so differ from the physical variables by terms involving the dispersion.

We have thus derived how these quantities are affected by coupling. We can now find some useful relationships among these quantities. First we have the obvious ones

$$
\boldsymbol{\epsilon}_{x, \mathrm{pr}}^{ \pm} \mp \boldsymbol{\epsilon}_{z, \mathrm{pr}}^{ \pm}=\left\langle g_{x}\right\rangle_{\mathrm{eq}}^{ \pm} \mp\left\langle g_{z}\right\rangle_{\mathrm{eq}}^{ \pm} .
$$

We can also show that

$$
\chi_{x} \epsilon_{x, \mathrm{pr}}^{-}+\chi_{z} \epsilon_{z, \mathrm{pr}}^{-}=\chi_{x} \epsilon_{x}+\chi_{z} \epsilon_{z}
$$

and

$$
\chi_{x} \epsilon_{x, \mathrm{pr}}^{+}-\chi_{z} \epsilon_{z, \mathrm{pr}}^{+}=\chi_{x} \epsilon_{x}-\chi_{z} \epsilon_{z} .
$$

Here, $\epsilon_{x, z}$ are the uncoupled emittances. These last two equations are only strictly true if the middle term in the global damping decrements is zero. However, this term will often be small due to the oscillatory nature of the integrand as we have discussed previously. Compare these sum rules to the sum rules for the equilibrium emittances of the eigenmodes near a sum/difference resonance [Eqs. (65)], one simply replaces $\chi_{1,2}$ with $\chi_{x, z}$ and $\left\langle g_{1,2}\right\rangle$ with $\epsilon_{x, z, \mathrm{pr}}$.

Finally, consider the equilibrium $\Sigma$ matrix near an integer/half-integer resonance. As noted with Eq. (65), one can think of the effect of the coupling as causing a perturbation to the lattice parameters. Thus, in computing $\Sigma$, one must take two effects into account: the perturbation to the lattice parameters, and the perturbation to the diffusion coefficient. The perturbed diffusion coefficients are given in Table IV, and the rest of the perturbed lattice parameters can be found in the same way as in the derivation of Eq. (259).

\section{PLOTS AND DISCUSSION}

We now have expressions for all the quantities needed to explore questions of stability and equilibrium beam distri-
TABLE VI. Parameters used in our numerical examples based on the PEP-II Low Energy Storage Ring.

\begin{tabular}{lc}
\hline \hline Parameter & Value \\
\hline $\mathrm{C}$ & $2199.33 \mathrm{~m}$ \\
$\alpha_{c}$ & $1.23 \times 10^{-3}$ \\
$\chi_{x}$ & $1.19 \times 10^{-4}$ \\
$\chi_{z}$ & $2.4 \times 10^{-4}$ \\
$\epsilon_{x}$ & $49.2 \times 10^{-9} \mathrm{~m}$ \\
$\epsilon_{z}$ & $9.35 \times 10^{-6} \mathrm{~m}$ \\
$\bar{d}_{x}$ & $2.34 \times 10^{-11} \mathrm{~m}$ \\
$\bar{d}_{z}$ & $8.98 \times 10^{-9} \mathrm{~m}$ \\
$\beta\left(s_{\text {cav }}\right)$ & $20 \mathrm{~m}$ \\
$\alpha\left(s_{\text {cav }}\right)$ & 0 \\
$\beta\left(s_{\text {crab }}\right)$ & $20 \mathrm{~m}$ \\
$\alpha\left(s_{\text {crab }}\right)$ & 0 \\
$\eta\left(s_{\text {cav }}\right)$ & $0-3 \mathrm{~m}$ \\
$\eta^{\prime}\left(s_{\text {cav }}\right)$ & 0 \\
$\eta\left(s_{\text {crab }}\right)$ & $0-3 \mathrm{~m}$ \\
$\eta^{\prime}\left(s_{\text {crab }}\right)$ & 0 \\
$\xi_{c}$ & $0-0.0031 / \mathrm{m}$ \\
\hline \hline
\end{tabular}

bution near each of the linear resonances for the two examples. We draw our parameters from Table VI which is based on the PEP-II storage ring. Damping rates are converted to $\chi_{x, z}$ by multiplying by the revolution period. Given the equilibrium emittances $\epsilon_{x}$ and $\epsilon_{z}$ we compute the integrated diffusion coefficients $\bar{d}_{x}$ and $\bar{d}_{z}$ using $\bar{d}_{x, z}=$ $4 \epsilon_{x, z} \chi_{x, z}$. Note the factor of 2 difference between the emittance and $\left\langle g_{x, z}\right\rangle_{\mathrm{eq}}$. For the sake of simplicity, we set $\alpha_{x}=\eta^{\prime}=0$ in all examples.

\section{A. Global quantities near sum/difference resonances}

For the sum and difference resonances, in Figs. 1 and 2, we plot the damping decrements $\chi_{1,2}$, the coupled integrated diffusion coefficients $\bar{d}_{1,2}$ and the resulting equilibrium invariants $\left\langle g_{1,2}\right\rangle_{\mathrm{eq}}$. In Figs. 3 and 4 , we plot the projected emittances, $\boldsymbol{\epsilon}_{x, z, \mathrm{pr}}$ which are given by Eqs. (287), (288), (290), and (291). From these quantities, one can determine the equilibrium transverse beam size and bunch length as given in Eqs. (295) and (296). In Figs. 5 and 6 we again plot the projected emittances from Figs. 3 and 4, but with 3D plots showing the full scale range.

\section{B. Antidamping instability}

An interesting effect that we have seen is that the damping decrement can become negative near a sum resonance when the motion is otherwise stable. This region of antidamping is larger, the larger the ratio of $\chi_{z}$ and $\chi_{x}$ [see Eq. (272)]. For the parameters used here, it is quite a small region on the $\nu_{x}-\nu_{z}$ plot for both of our examples. A natural way to affect this antidamping region is to control the ratio 

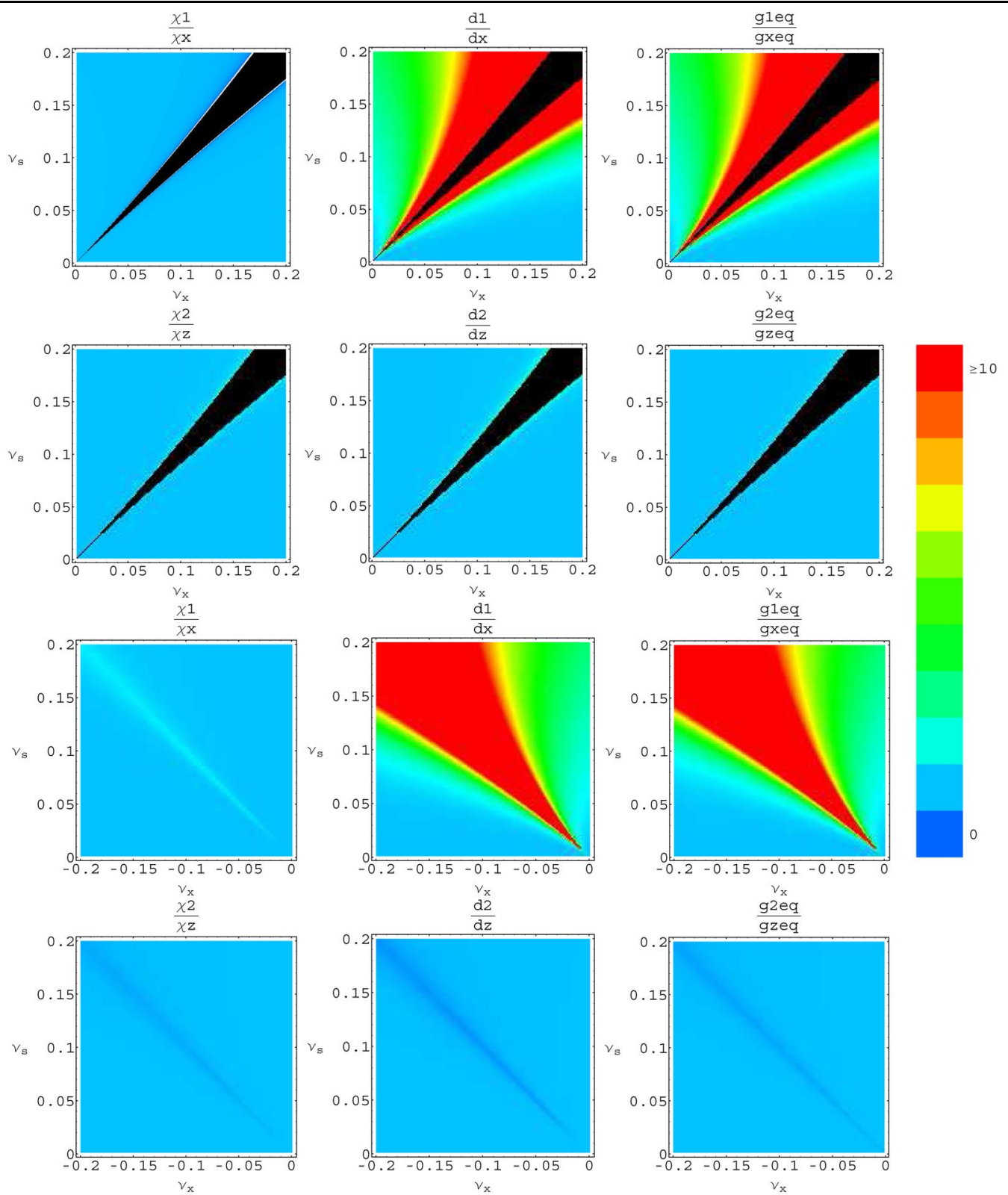

FIG. 1. (Color) In this figure, we plot global damping, diffusion, and equilibrium invariant values for coupling due to a dispersion of $1 \mathrm{~m}$ at an rf cavity near the sum/difference resonances. The upper two rows are the sum resonance, and lower two are the difference resonance. The parameters are otherwise drawn from Table VI based on the PEP-II LER. The quantities are plotted as a function of the betatron tune $\nu_{x}$ and the synchrotron tune $\nu_{s}$ which is positive and equal to $-\nu_{z}$, thus giving an inversion of sum and difference resonances. $\chi_{1}$ and $\chi_{2}$ are global damping decrements expressed in Table V. $\bar{d}_{1}$ and $\bar{d}_{2}$ are global diffusion coefficients expressed in Table IV, and $\left\langle g_{1}\right\rangle_{\mathrm{eq}}$ and $\left\langle g_{2}\right\rangle_{\mathrm{eq}}$ are one half the ratio of these quantities as given by Eq. (47). All quantities have been divided by their uncoupled values so that the blue region with the value of 1 represents no effect from coupling. The region of instability due to the Hamiltonian dynamics is black. There is also an extremely small region of antidamping instability outside the symplectic instability region for the sum resonance where the damping decrement $\chi_{1}$ is negative. This region is indicated by white.

$\chi_{z} / \chi_{x}$ through the damping partition number $\mathcal{D}$ as we see from Eqs. (108) and (109),

$$
\frac{\chi_{z}}{\chi_{x}}=\frac{\mathcal{J}_{z}}{\mathcal{J}_{x}}=\frac{2+\mathcal{D}}{1-\mathcal{D}}
$$

One can see from the values of $\chi_{x, z}$ in Table VI, that $\mathcal{D}$ is quite small with $\mathcal{D}=0.0056$. To explore the effect of changing $\mathcal{D}$ on the antidamping instability, we multiply $\chi_{x}$ by $1-\mathcal{D}$ and $\chi_{z}$ by $(2+\mathcal{D}) / 2$ and plot $\chi_{1}$ for rf cavity dispersion and a crab cavity for various values of $\mathcal{D}$. The results are given in Fig. 7 for $\mathcal{D}=0.25,0.5$, and 0.75. The Hamiltonian dynamics instability is colored black, whereas the antidamping instability is colored white. 

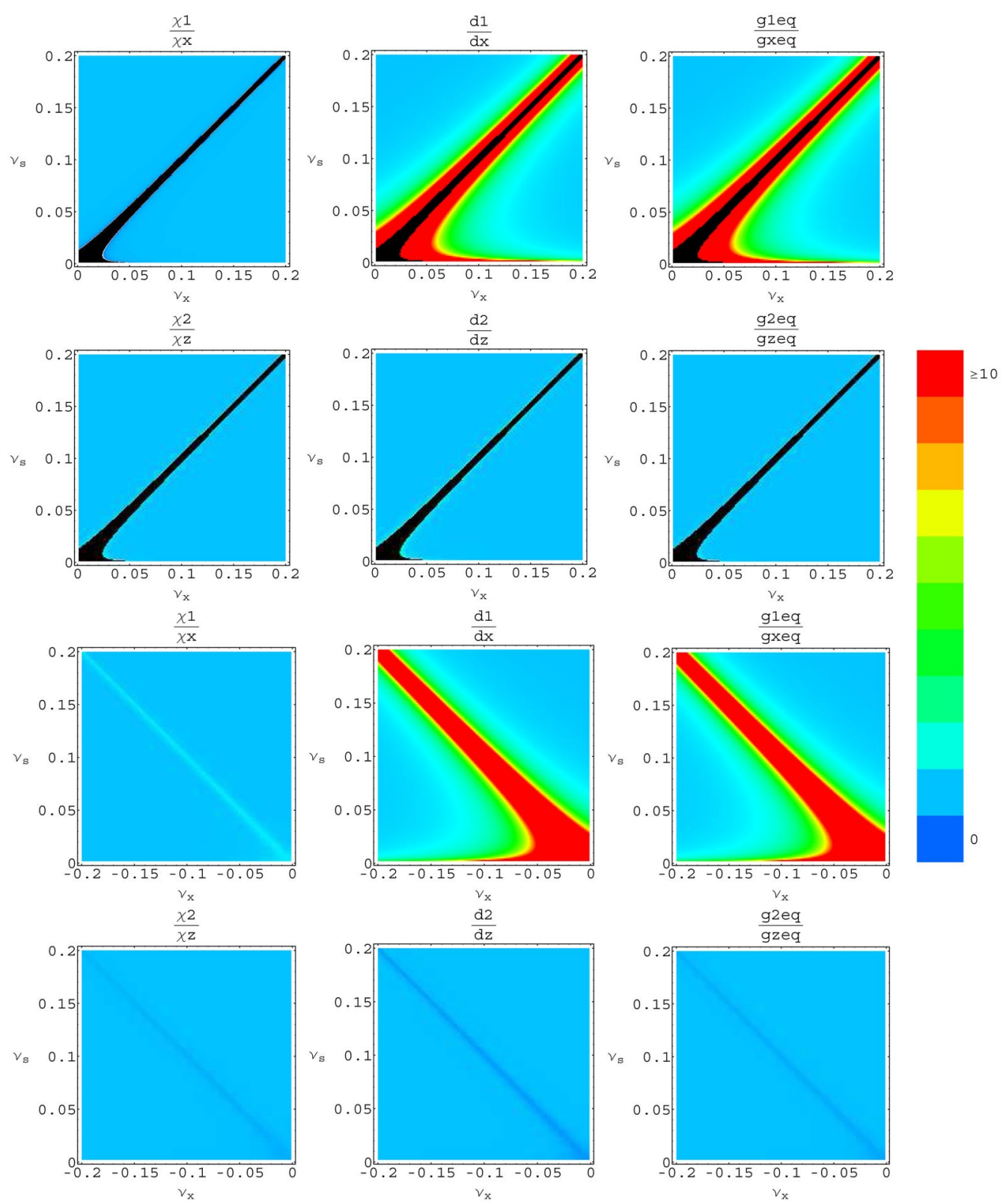

FIG. 2. (Color) In this figure, we plot global damping, diffusion, and equilibrium invariant values for coupling due to a crab cavity with $\xi_{c}=0.003$ near the sum and difference resonances. The dispersion at the crab cavity is set to 0 in this example. Because the coupling strength $\xi$ is inversely proportional to $\sqrt{\nu_{s}}$, the instability broadens for smaller $\nu_{s} \cdot \chi_{1}, \chi_{2}, \bar{d}_{1}, \bar{d}_{2},\left\langle g_{1}\right\rangle$ and $\left\langle g_{2}\right\rangle$ are the same as in Fig. 1 . As in Fig. 1, the small region of antidamping for $\chi_{1}$ near the sum resonance is colored white.

\section{Dispersion at crab cavity, numerical results}

Dispersion at the crab cavity affects all of the resonances. However, for the crab cavity near the sum and difference resonances, in our plots, we have set the dispersion to zero. For the case of $\nu_{x}$ near a half-integer, a case of particular interest, we have explored the effect of dispersion. In Fig. 8, we plot stability diagrams (stable if $|\lambda|=1$, unstable otherwise) for the cases of no dispersion and a dispersion of $3 \mathrm{~m}$. We see that when the dispersion is turned on, the integer and half-integer $\nu_{x}$ resonances become visible, as well as a shifting of the integer $\nu_{z}$ reso- nance. We have also plotted the equilibrium horizontal emittance near the $\nu_{x}$ half-integer resonance as well as the equilibrium longitudinal emittance near the $\nu_{z}$ integer resonance. For the $\nu_{x}$ half-integer resonance, since the width is largely independent of $\nu_{s}$, but depends on the dispersion, we have plotted the horizontal emittance as a function of the tune $\nu_{x}$ and the dispersion $\eta$, showing a broadening effect for larger dispersion.

Finally, we include an example of a numerical comparison between the eigenvalues obtained using our perturbation theory against those obtained numerically directly 

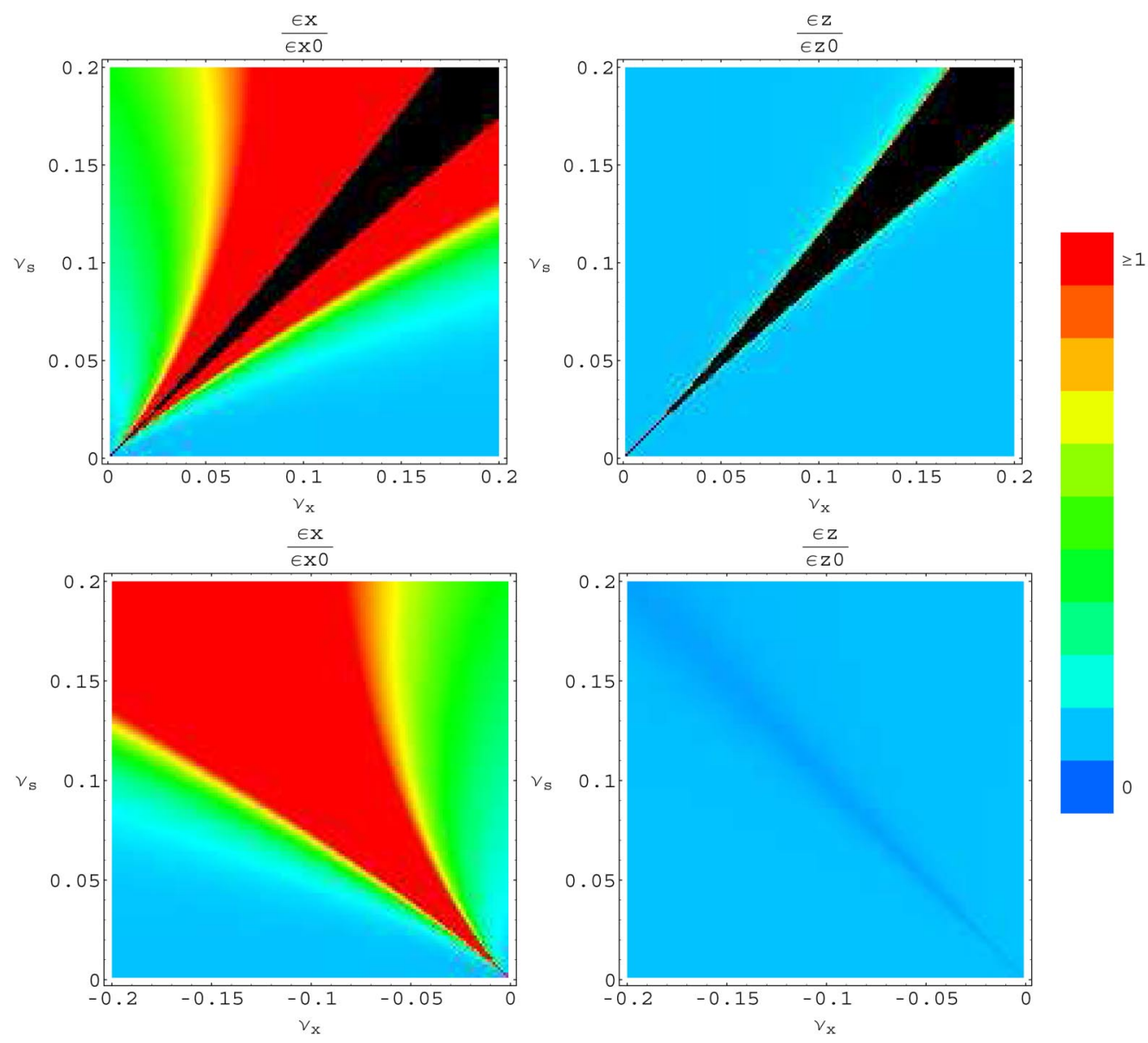

FIG. 3. (Color) Projected emittances near the sum and difference resonances due to dispersion at the rf cavity. The parameters are the same as those in Figs. 1. The upper two plots are for the sum resonance with $\epsilon_{x, \text { pr }}^{+}$and $\epsilon_{z, \text { pr }}^{+}$defined in Eqs. (287) and (288). The lower two plots are for the difference resonance with $\epsilon_{x, \mathrm{pr}}^{-}$and $\epsilon_{z, \mathrm{pr}}^{-}$defined in Eqs. (290) and (291).

from the one-turn matrix. In Fig. 9 we plot the computed versus numerical values of $\mu_{2}$ near the $\nu_{z}$ integer resonance, as a function of $\nu_{x}$ including a dispersion function of $0.1 \mathrm{~m}$ at the crab cavity. The agreement is quite reasonable.

\section{Instabilities near half-integer $\boldsymbol{\nu}_{\boldsymbol{x}}$}

We are particularly interested in the case of the betatron tune just above the half-integer because this is a case that is commonly used in colliders such as PEP-II and KEK-B. This means that we should pay attention to the $\nu_{x}$ halfinteger resonance. We see that there is indeed such an instability for a dispersive rf cavity and for a crab cavity. For the dispersive if cavity if we set $\alpha_{x}=\eta^{\prime}=0$ in Eq. (219), we find an instability for

$$
\frac{1}{2}<\nu_{x}<\frac{1}{2}+\frac{1}{2 \pi} \frac{\eta^{2}}{C \alpha_{c} \beta_{x}} \mu_{s}^{2}
$$

For the crab cavity, we also find a $\nu_{x}$ half-integer resonance. The condition is given by Eq. (245). When the dispersion at the crab cavity is negligible, the condition is

$$
\frac{1}{2}<\nu_{x}<\frac{a \beta_{x} \xi_{c}^{2}}{8 \pi} \quad \text { (no dispersion). }
$$

This is typically quite a narrow instability. When dispersion dominates, the condition becomes

$$
\left|\nu_{x}-\frac{1}{2}\right|<\frac{\eta \xi_{c}}{2 \pi} \quad \text { (dispersion dominates). }
$$

In this case, near $\nu_{x}=1 / 2$, applying Eq. (284), and using $\Delta \mu \approx 2\left(\mu_{x}-\pi\right)$, the emittance will be increased by a factor of

$$
\frac{\epsilon_{1}}{\epsilon_{x}}=\frac{1}{\sqrt{1-\left(\frac{\eta \xi_{c}}{2 \pi\left(\nu_{x}-1 / 2\right)}\right)^{2}}} .
$$

As an example, for the parameters in Table VI with a dispersion of $1 \mathrm{~m}$ at the crab cavity, when $\nu_{x}-1 / 2$ is 0.0016 , there is an increase in emittance of $10 \%$ due to this resonance.

In addition to the $\nu_{x}$ half-integer resonance, because the synchrotron tune is typically small, one is also concerned with the $\nu_{z}$ integer resonance. In fact, this resonance is 

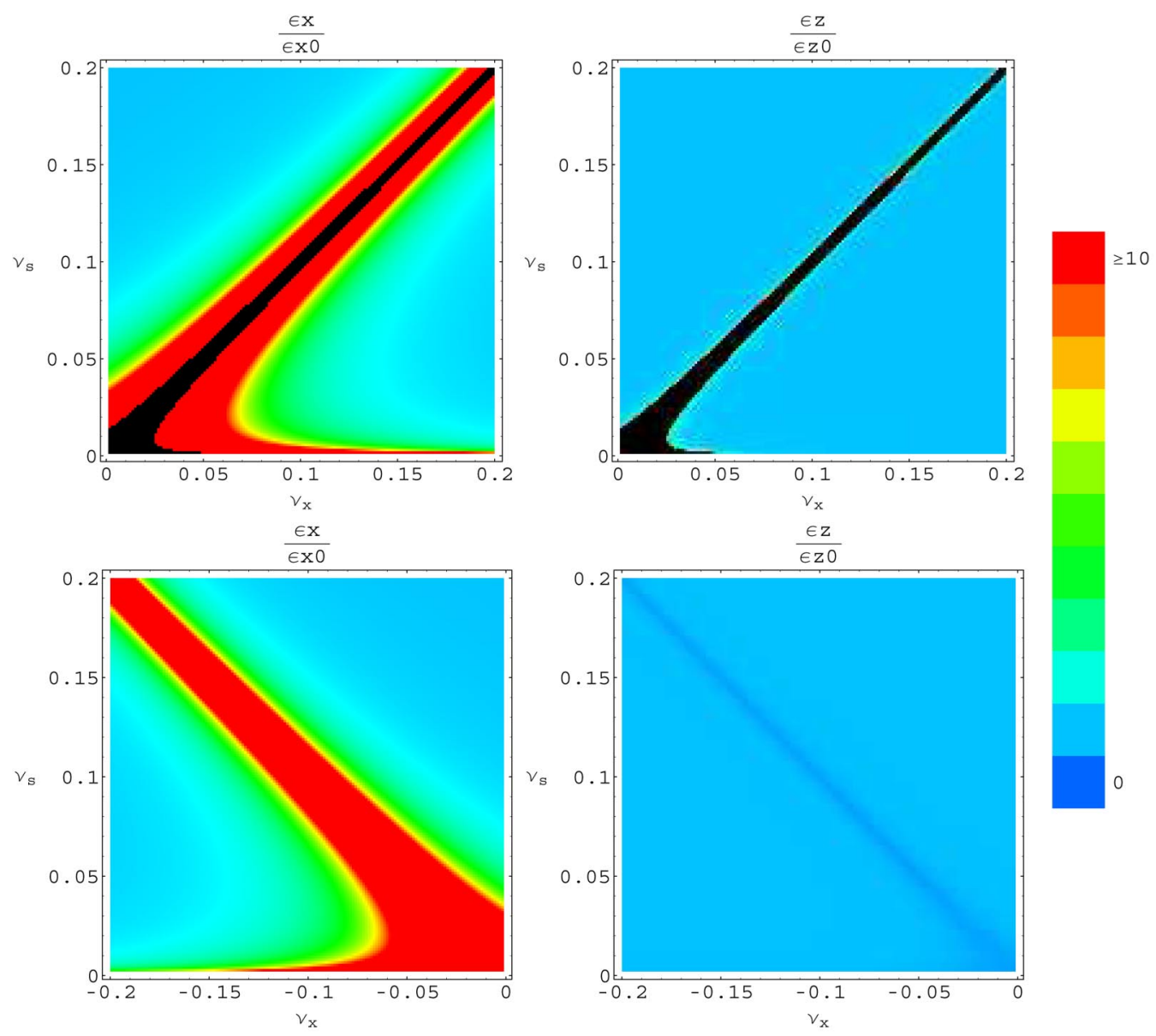

FIG. 4. (Color) Projected emittances near the sum and difference resonances due to a crab cavity. The four plots have the same meanings as in Fig. 3. The parameters are the same as those in Figs. 2.

explored in [5]. Looking at Eq. (D25), because of the factor $\cot \frac{\mu_{x}}{2}$ we expect an instability for this resonance, although very near $\nu_{x}=\frac{1}{2}$, it is quite narrow. Adding dispersion changes this simple picture. As we see in Fig. 7, the dispersion moves the $\nu_{z}=0$ resonance into the region $\nu_{x}<\frac{1}{2}$, and for a fixed value of $\nu_{x}$ increases its width in $\nu_{s}$. The perturbed synchrotron tune due to the integer $\nu_{z}$ resonance is given by

$$
\nu_{2}=\sqrt{\nu_{s}^{2}+\left(\frac{C \alpha_{c} \beta_{x}}{4} \cot \left(\pi \nu_{x}\right)-\eta^{2}\right)\left(\frac{\xi_{c}}{2 \pi}\right)^{2}}
$$

from which we see that for $\nu_{x}$ just above $\frac{1}{2}$, there can be an instability when

$$
\nu_{s}<\frac{\xi_{c}}{2 \pi} \sqrt{\frac{C \alpha_{c} \beta_{x}}{4}\left(\nu_{x}-\frac{1}{2}\right)+\eta^{2}},
$$

which was also derived in [5]. For $\nu_{x}<\frac{1}{2}$, we see that this resonance can substantially increase the synchrotron tune.

Associated with this integer $\nu_{z}$ resonance is an increase or decrease of longitudinal emittance. Applying Eq. (285), and considering the case where the dispersion at the crab cavity is zero, we find that the longitudinal emittance is multiplied by a factor of

$$
\frac{\epsilon_{2}}{\epsilon_{z}}=\frac{\nu_{s}+\left[1+\operatorname{sgn}\left(\nu_{x}-\frac{1}{2}\right)\right] \frac{C \alpha_{c} \beta_{x}}{4 \nu_{s}} \cot \left(\pi \nu_{x}\right)\left(\frac{\xi_{c}}{2 \pi}\right)^{2}}{\sqrt{\nu_{s}^{2}+\frac{C \alpha_{c} \beta_{x}}{4} \cot \left(\pi \nu_{x}\right)\left(\frac{\xi_{c}}{2 \pi}\right)^{2}}} .
$$

We have used Eq. (162) and the fact that $\Delta \mu \approx 2 \mu_{z}$ for the integer $z$ resonance. The sgn (step) function comes from the $\cos \phi$ term in (162), where $\phi$ involves $\cot \left(\mu_{x} / 2\right)$ as we find from Table III. Note that the sgn function does not make this expression discontinuous at $\nu_{x}=1 / 2$, since $\cot (\pi / 2)=0$ and so there is no perturbation there.

One may also be interested to know the effects of dispersion at an rf cavity when the $\nu_{x}$ is near a half-integer. For this case, we find a very weak $\nu_{x}$ half-integer stop band. This instability occurs when

$$
\frac{2 \pi \mathcal{H}_{x}}{C \alpha_{c}} \nu_{s}^{2}>\nu_{x}-\frac{1}{2}
$$

which is a small stop band both because of the $\nu_{s}^{2}$ and the $\mathcal{H}_{x}$ which is $\eta_{c}^{2} / \beta_{x}$ when $\eta^{\prime}=\alpha_{x}=0$, where $\eta_{c}$ is the 

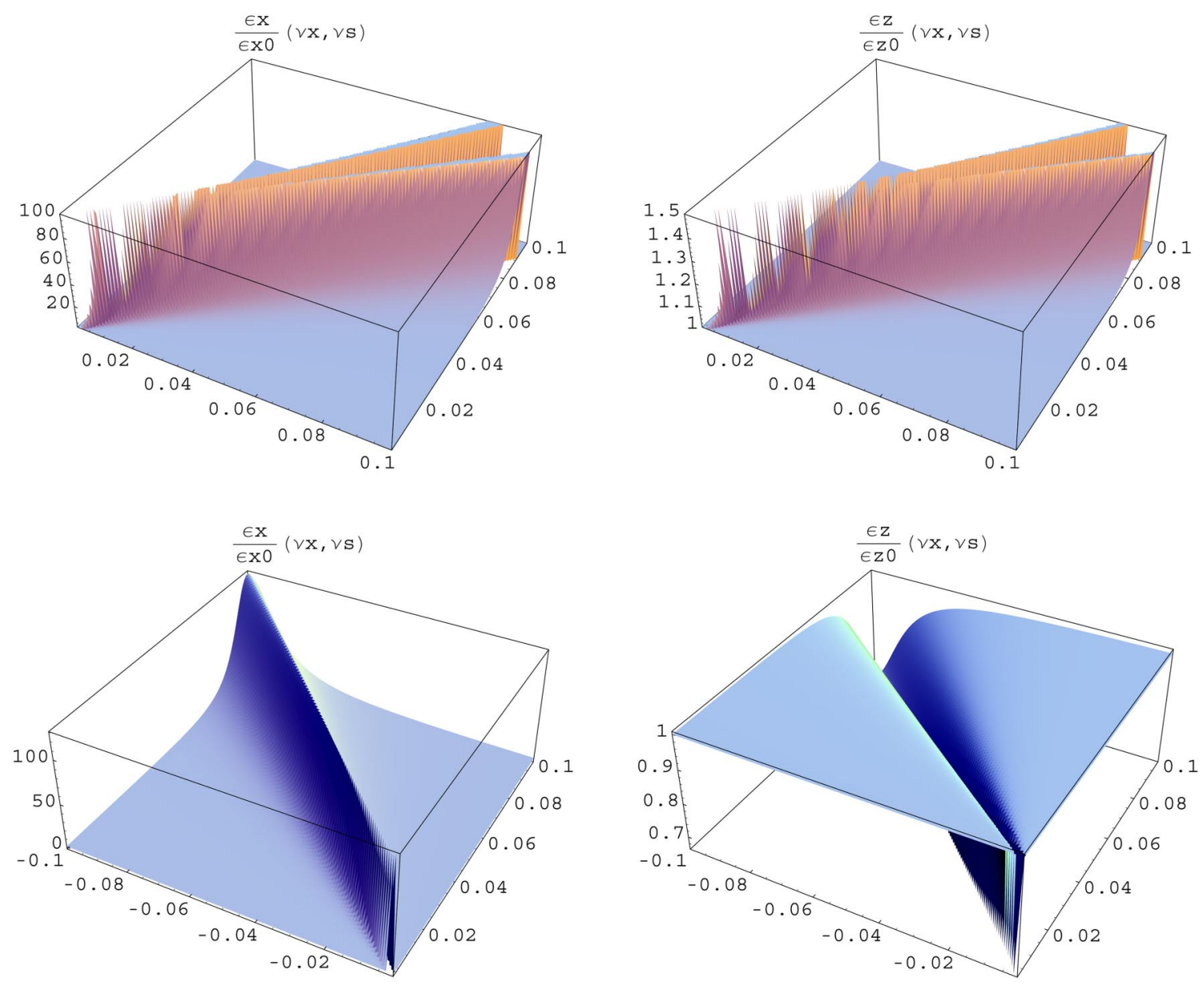

FIG. 5. (Color) 3D plots of projected emittances near the sum and difference resonances due to a dispersive rf cavity. The four plots have the same meanings as in Fig. 3. The parameters are the same as those in Figs. 1.

dispersion at the rf cavity. Regarding the $\nu_{z}$ integer resonance, we find that it is absent for coupling due to a dispersive rf cavity. One can understand this fact by observing that the strength of the perturbation due to dispersion is proportional to $\nu_{s}^{2}$, so approaching $\nu_{s} \rightarrow 0$ effectively turns off the perturbation and avoids an instability.

Even when there is not an instability, we have seen that the emittances can be affected near a resonance. For the case of the $\nu_{x}$ half-integer resonance, the horizontal emittance can become large, whereas for the $\nu_{z}$ integer resonance, the longitudinal emittance (and correspondingly bunch length and energy spread) can become large. For the case of small synchrotron tune and betatron tune just above the half-integer a combination of these two effects is expected, i.e., a combination of the bottom two plots in Fig. 8.

\section{E. Instabilities near integer $\boldsymbol{\nu}_{\boldsymbol{x}}$}

In the case of a storage ring operated near an integer $\nu_{x}$, one needs to be concerned with four resonances: integer $\nu_{x}$ and $\nu_{z}$, and the sum or difference resonances. Refer to the upper two plots in Fig. 8 for a numerical example of the instability region. From our analytical expressions for the perturbed tunes, we learn that for small $\nu_{s}$, a crab cavity is particularly dangerous in this region because the effective coupling strengths for the sum and difference resonances $\xi_{ \pm}$scales as $1 / \sqrt{\nu_{s}}$. Regarding the integer $\nu_{z}$ resonance, $\nu_{x}$ above an integer is safer than below an integer because the $\nu_{z}$ integer resonance has a particularly large stop band just below integer $\nu_{x}$ coming from the factor of $\cot \left(\pi \nu_{x}\right)$ in Eq. (189). For $\nu_{x}$ above an integer, this factor causes increased stability, even reducing equilibrium longitudinal emittance, as seen in the dark blue lower left region of the bottom left plot of Fig. 8. However, because of the strong $\nu_{x}=-\nu_{z}$ sum resonance, this effect would be washed out, at least for $\nu_{x}$ near the integer. Nevertheless, this result suggests the possibility of using coupling to increase stability and reduce emittance through careful tuning near a coupling-induced integer or half-integer resonance, perhaps in another context such as transverse coupling.

\section{F. Single versus multiple resonances}

In this paper, we have analyzed each resonance in isolation. This should be accurate as long as the tunes are such 

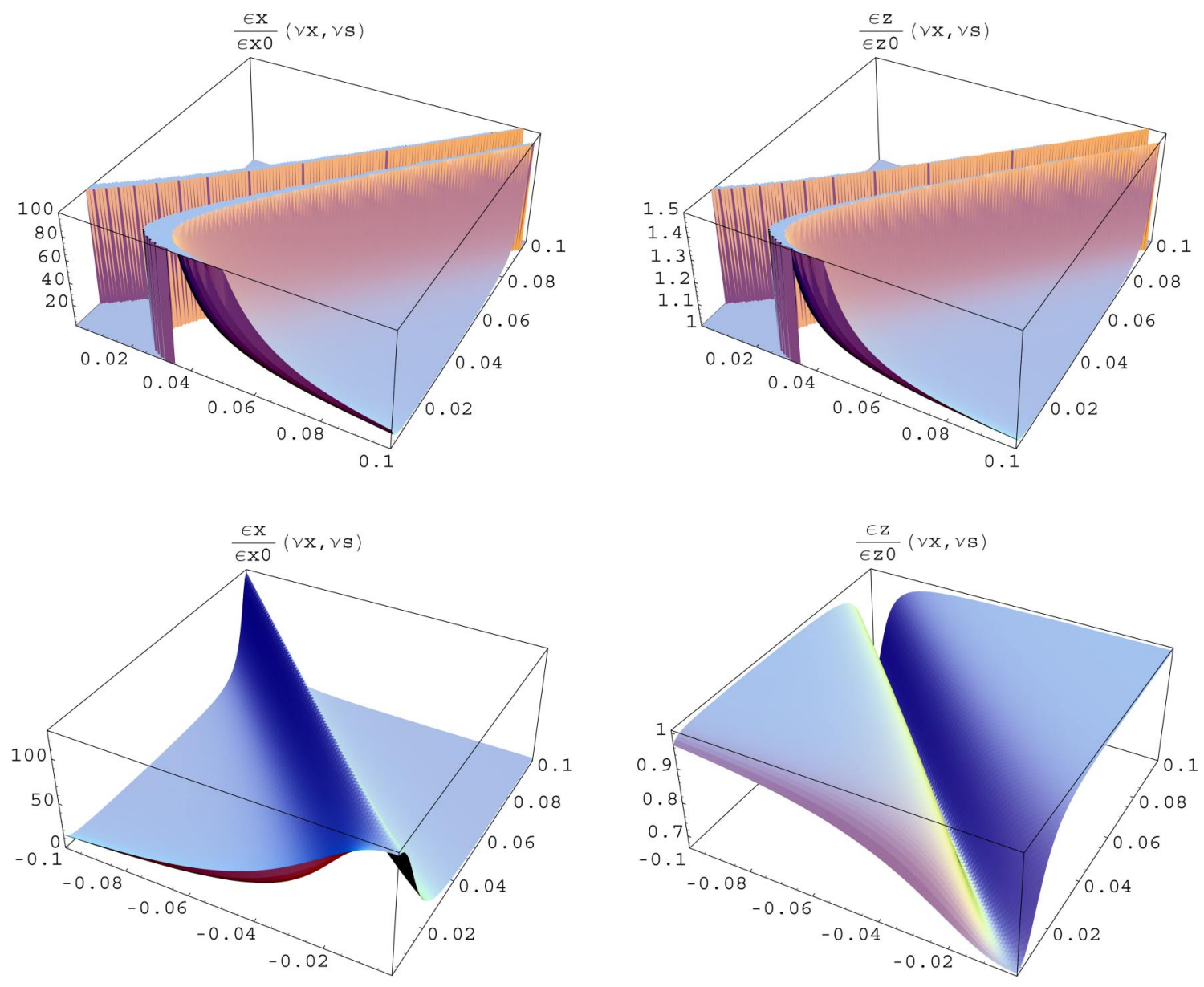

FIG. 6. (Color) 3D plots of projected emittances near the sum and difference resonances due to a crab cavity. The four plots have the same meanings as in Fig. 3. The parameters are the same as those in Figs. 2.

that they are much closer to one resonance than any other. Particularly near $\nu_{x}=\nu_{s}=0$ this can break down. In this situation, the system can be near three resonances, simultaneously: the integer $\nu_{x}$, the integer $\nu_{z}$, and either the sum or difference resonance. In this case, we expect that there may be greater errors in our expressions. In the case that one of the resonances does not cause an instability, but still can strongly affect the tune, such as for the difference resonance or the coupling $\nu_{z}$ integer resonance for $\nu_{x}<$ $\frac{1}{2}$, one could use the perturbed, but not unstable tune as an input to determine the instability of the other tune. When the tunes are equally near to multiple resonances, a more elaborate analysis is necessary. We note that our perturbation theory can accommodate this situation (e.g., the quadruple degeneracy near $\nu_{x}=\nu_{z}=0$ ) but we do not pursue it further here.

\section{CONCLUSION}

In this paper, we have shown a way to analyze the effects of coupling near linear resonances. If we focus on 4D phase space, there are in general six such resonances to be concerned about: the sum and difference resonances along with the two integer $\left(\nu_{x}, \nu_{z}\right)$ and two half-integer $\left(\nu_{x}, \nu_{z}\right)$ resonances. For the case of synchrobetatron coupling, $\nu_{z}$ is typically much less than $\frac{1}{2}$ and so there are really five resonances to consider.

In the presence of damping and diffusion mechanisms, such as synchrotron radiation, even near to such resonances, we have argued that the beam distribution can usually be described as a Gaussian function of the invariants of the one-turn map. Regarding the effect of coupling on this near resonance distribution, one needs to consider both the issues of stability and the effect on the equilibrium emittances.

Instability can occur in one of two ways. First, the coupling can cause one or two pairs of the eigenvalues to become complex, which for a symplectic matrix means that there will be a growing mode. Second, near a sum resonance, the coupling can mix together the damping rates in such a way that one of the modes has negative damping, which also indicates an instability.

Even if instability can be avoided, coupling near a resonance can have a substantial effect on the equilibrium 

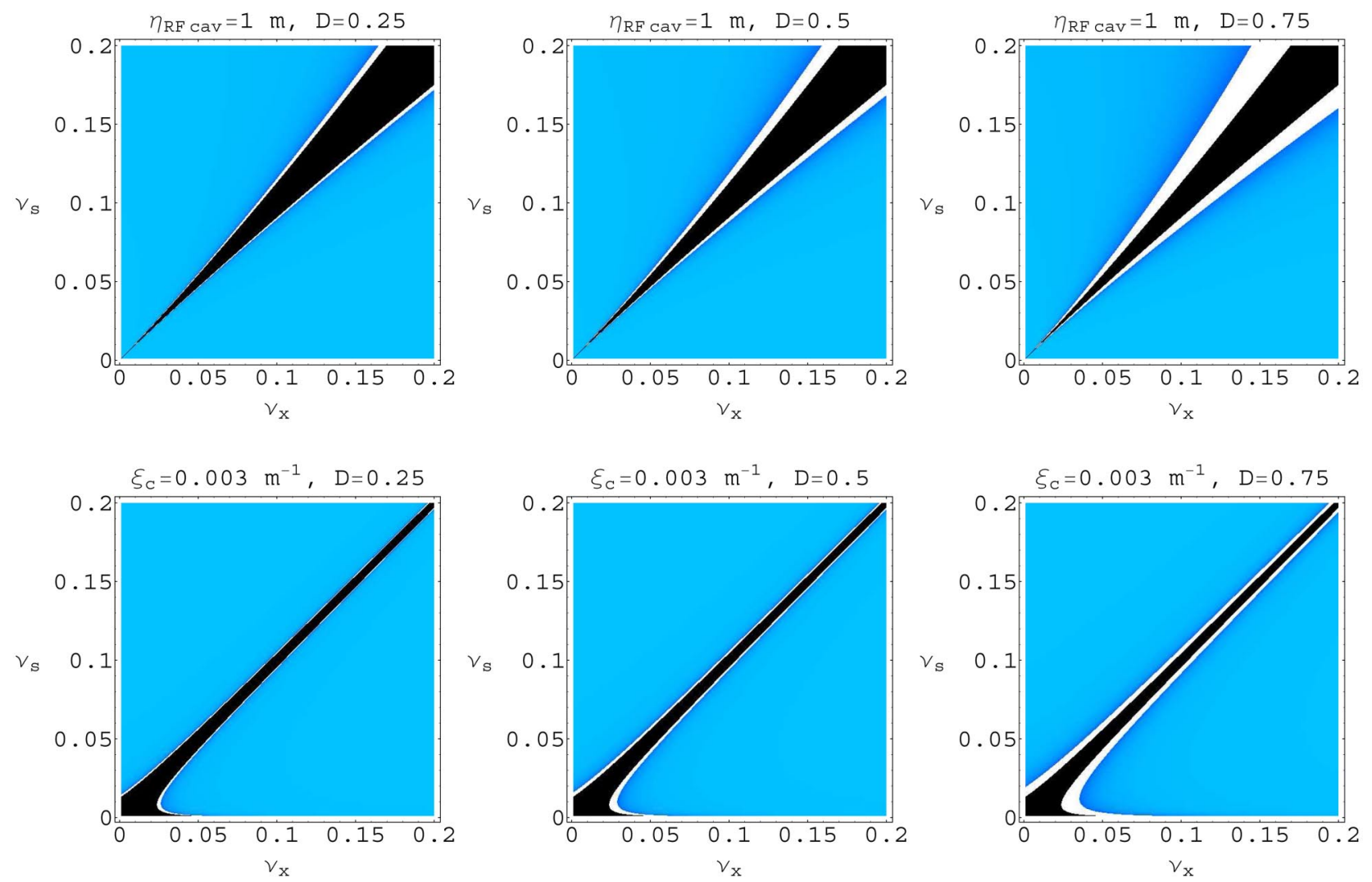

FIG. 7. (Color) Damping decrement near sum resonance for coupling due to a dispersive rf cavity (upper plots) and a crab cavity (lower plots). The black region represents an instability in the Hamiltonian dynamics, whereas the white area represents a negative value of $\chi_{1}$ and hence an antidamping instability. We vary the damping decrement $\mathcal{D}$. In the two leftmost plots it is 0.25 . For the center plots it is 0.5 , and the right plots have 0.75 . The plots of $\chi_{1}$ near the sum resonance in Figs. 1 and 2 plot the same quantities, except that in those cases, $\mathcal{D} \approx 0$ as discussed in the text. Increasing $\mathcal{D}$ increases the ratio of $\chi_{z}$ and $\chi_{x}$ which widens the region where an antidamping instability occurs.

emittances. This is particularly so if there is a large discrepancy between the emittances, which is indeed the typical case for synchrobetatron dynamics in which the horizontal emittance is much smaller than the longitudinal. For the case of PEP-II, $\epsilon_{z} / \epsilon_{x}=190$. Intuitively, one might expect, therefore, that synchrobetatron coupling would have a major effect on transverse emittance, since it is coupled to such a huge reservoir of longitudinal emittance. This is indeed what we find for both the sum and difference resonances. Near a sum resonance, although both emittances blow up at the resonance, a little bit off resonance one finds a large growth in the horizontal emittance $\epsilon_{x}$ and a very small growth in the longitudinal emittance $\epsilon_{z}$. Near a difference resonance, we also find major growth in $\epsilon_{x}$ whereas in this case there is a small decrease in $\epsilon_{z}$. The relationships between the emittances and how a change in one affects a change in the other can be understood by looking at the various sum rules we have derived in Sec. II D. We have also derived [Eqs. (298) and (299)] sum rules for how the projected emittances relate to each other. We find qualitatively similar behavior for both the projected emittances and the emittances of the eigenmodes.
One might generalize the intuitive concept of coupling as a sort of equilibration between reservoirs and roughly state that when there is a large discrepancy between two quantities, coupling can have a strong effect near a resonance. We have just mentioned this effect for the case of the equilibrium emittances near sum/difference resonances. The other example where this is evident is in the previously mentioned antidamping instability near a sum resonance. We have found that the larger the ratio of the damping rates, the larger the region of tune space that becomes unstable for a given coupling strength. A natural way in which this ratio is altered is through the damping partition number $\mathcal{D}$. We have seen that increasing $\mathcal{D}$ expands this region of the antidamping instability.

It turns out, however that the intuition of the previous paragraph does not apply near integer and half-integer resonances. Although coupling can cause an instability when one of the tunes is near an integer or half-integer, the damping rates are not affected much by this instability and although the diffusion coefficient for the unstable mode does get large and diverge at the instability, it does not mix with the other (stable mode) diffusion coefficient. Thus, although emittance is still affected by being 

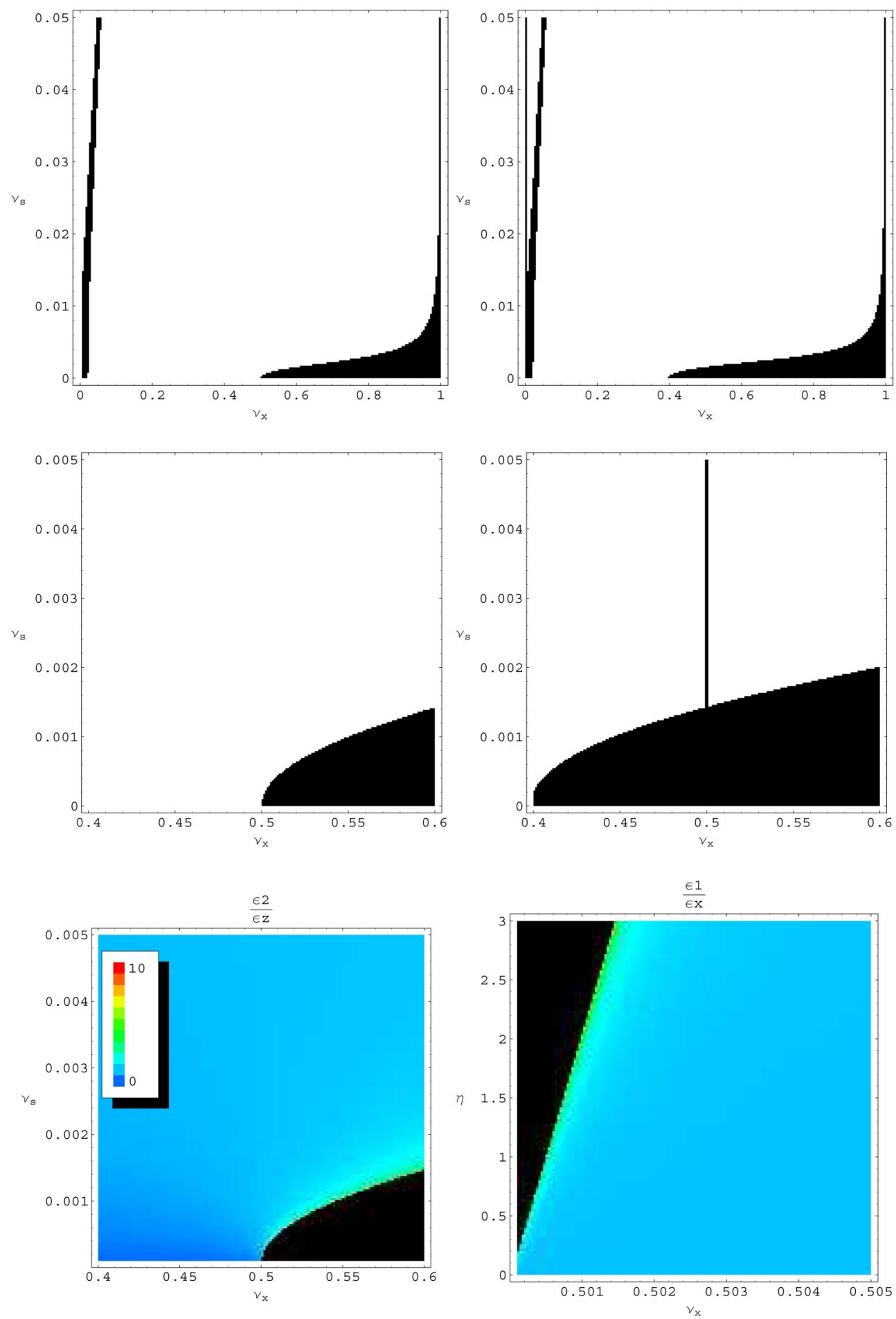

FIG. 8. (Color) These six plots relate to crab cavity coupling. The upper four plots are instability diagrams. The eigenvalues $\lambda_{j}$, have been computed numerically for each value of the tunes and if for each $j,\left|\lambda_{j}\right|=1$, it is stable and colored white, whereas $\left|\lambda_{j}\right| \neq 1$ is unstable and colored black. The left plot has no dispersion at the crab cavity, while the right plot has a dispersion of 3 meters. The middle two plots show a magnified view of the upper two plots near $\nu_{x}=\frac{1}{2}$. Note that the $\nu_{x}$ half-integer resonance caused by dispersion is only visible upon magnification. The integer $\nu_{x}$ resonance also appears with dispersion as can be seen from the thin black line near $\nu_{x}=0$ in the upper right plot. The bottom two plots give the horizontal and longitudinal emittance growth factors defined in Eq. (47). Because $\chi_{1,2}=\chi_{x, z}$ for integer and half-integer resonances, this is also given by the ratios $\bar{d}_{1} / \bar{d}_{x}$ and $\bar{d}_{2} / \bar{d}_{z}$ where $\bar{d}_{1}$ and $\bar{d}_{2}$ are defined in Table IV. Explicit expressions for the emittance growth for transverse and longitudinal are given in Eqs. (304) and (307). We have set the dispersion at the crab cavity to zero here. The bottom left is plotted as a function of $\nu_{x}$ and $\nu_{s}$, while the bottom right is plotted as a function of $\nu_{x}$ and dispersion at the crab cavity in units of meters. The $\nu_{x}$ half-integer instability does not depend on $\nu_{s}$. 


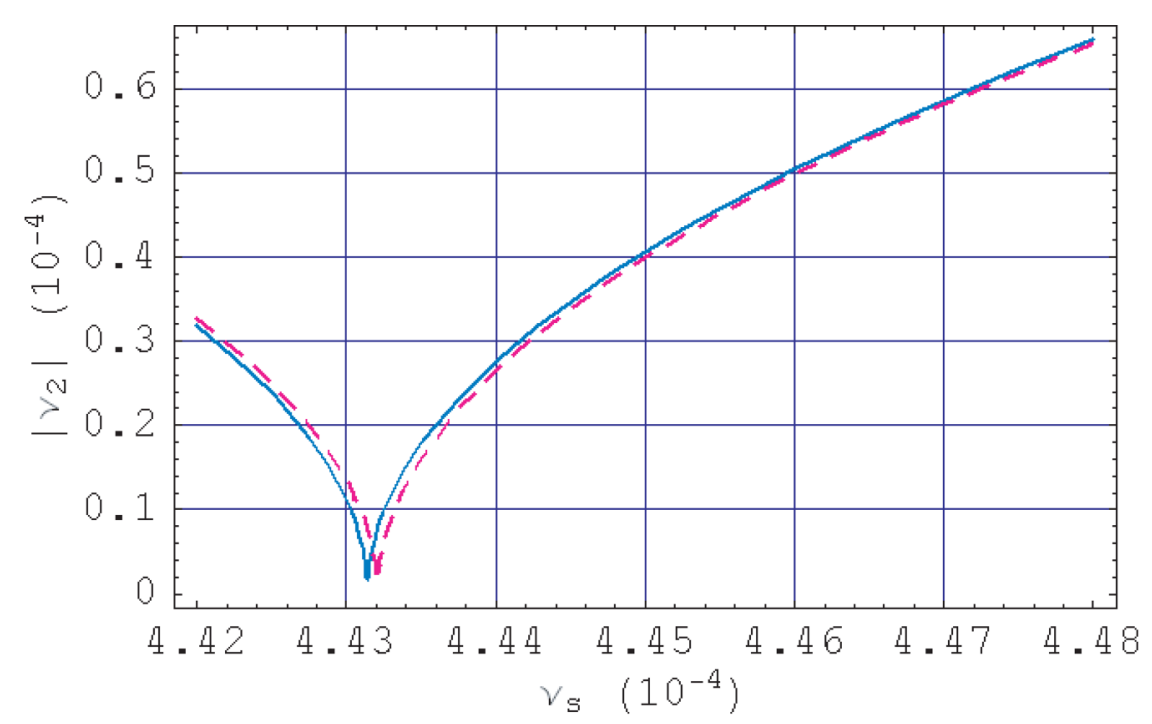

FIG. 9. (Color) Comparison of eigenvalue formula to direct numerical calculation. Here, $\nu_{x}=0.51$, and we plot the absolute value of $\nu_{2}=\mu_{2} / 2 \pi$, which becomes imaginary (unstable) due to the integer $\mu_{s}=0$ resonance. We have set the dispersion at the crab cavity to be $\eta=0.1 \mathrm{~m}$. The dashed red curve is the numerical result, while the blue is Eq. (249).

near an integer or half-integer resonance, it is not as strong of an effect as with the sum and difference resonances where disparity between transverse and longitudinal damping and diffusion rates and emittances enhances the impact.

We have derived a perturbation theory which gives expressions for the coupled damping and diffusion coefficients and equilibrium eigeninvariants and projected emittances in the vicinity of the resonance. The key to this effort was to take the degenerate one-turn map as the unperturbed starting point and treat both coupling and difference from resonance as perturbations. Once the matrix elements of the perturbation are properly defined, the results are quite similar to those for finding perturbed energies and eigenfunctions in degenerate perturbation theory in quantum mechanics. The sign involved in the definition of the "bra" $v^{j}$ [Eq. (16)] distinguishes stable from unstable resonances: degeneracies involving two positive or negative modes are stable, whereas degeneracies involving one negative and one positive mode are unstable. Thus, one finds stability at a difference resonance $\left(\nu_{x}=\nu_{z} \bmod 2 \pi\right)$ and instability at a sum $\left(\nu_{x}=-\nu_{z} \bmod \right.$ $2 \pi)$, integer $\left(\nu_{x, z}=-\nu_{x, z}=0 \bmod 2 \pi\right)$ and half-integer $\left(\nu_{x, z}=-\nu_{x, z}=1 / 2 \bmod 2 \pi\right)$ resonance.

One might be surprised to find out that coupling can in fact cause an instability at an integer or half-integer resonance. A pure coupling effect, as we have seen in the dispersion free crab cavity, mixes transverse and longitudinal dynamics: the perturbation has no elements in the on-diagonal two by two blocks. Nevertheless, a careful analysis, involving second order degenerate perturbation theory, allowed the calculation of the width of this instability and the effect on damping and diffusion. It has the unusual property that whether or not a given tune will go unstable depends on the value of the other (stable) tune.

An important example of a coupling-caused integer instability is the integer resonance for the synchrotron tune $\nu_{s}$. Because orf systems naturally produce a small $\nu_{s}$, this resonance (near $\nu_{s}=0$ ) is always a possibility. We find that for $\nu_{x}>1 / 2$ coupling can cause an instability for small enough $\nu_{s}$. For the case of dispersion at an rf cavity, we found that this instability was not present. In the case of a crab cavity, however, we do indeed have to deal with this instability. As we have discussed, we can understand this difference in behavior of the two coupling sources by noting that coupling due to rf cavity dispersion gets weaker for small $\nu_{s}$ whereas crab cavity coupling tends to get stronger at small $\nu_{s}$.

This work was motivated in part by planned installation of crab cavities in colliders such as KEK-B. To minimize beam-beam effects, such colliders are typically operated with a betatron tune $\nu_{x}$ just above a half-integer. Although this is in the region where an instability due to the $\nu_{s}$ integer resonance is possible, the stop-band width at $\nu_{x}=$ $1 / 2$ is zero, growing with increasing $\nu_{x}$. This picture is complicated somewhat by the addition of dispersion that moves the onset of instability to $\nu_{x}<1 / 2$ and increases the stop-band width in $\nu_{s}$ for a fixed value of $\nu_{x}$ as we have discussed.

In addition to this $\nu_{s}=0$ resonance, operating near the half-integer $\nu_{x}$ raises the issue of the $\nu_{x}$ half-integer resonance. We have found that the crab cavity does cause an instability for $\nu_{x}$ near a half-integer, but it is relatively weak, with a width of $\eta \xi_{c}$ where $\eta$ is the dispersion at the crab cavity and $\xi_{c}$ is the crab cavity strength.

We can conclude that for $\nu_{x}$ near a half-integer, the synchrobetatron resonances caused by a crab cavity are 
relatively benign, although if $\nu_{x}$ is too close to the halfinteger and/or $\nu_{s}$ too close to zero, the dispersion at the crab cavity may need to be minimized to maintain safe operations and preserve small emittances.

As we have pointed out, near integer and half-integer coupling instabilities, there is no "mixing" effect on the emittances, as we see for the case with the sum/difference resonance: the large disparity between horizontal and longitudinal emittances does not contribute to an increased coupling effect. From this perspective, it is further seen that operating near half-integer $\nu_{x}$ is preferable to integer $\nu_{x}$. When $\nu_{x}$ is near an integer, because $\nu_{s}$ is small, one must also contend with the sum or difference resonances which can have major effects on horizontal emittance.

We have focused on the case of synchrobetatron coupling, giving details for a single crab cavity and a dispersive rf cavity. The same procedure applies to other cases of synchrobetatron coupling as well as for transverse betatron coupling. For more details of the transverse coupling case, see [15].

In cases where the convenient starting point is already coupled, one can still apply this analysis by working in coordinates that remove this coupling. This is essentially what we have done with dispersion. By using betatron coordinates, we are able to start from an uncoupled map. Thus, in a general situation, one would simply replace the matrix $\mathcal{B}$ with a more general decoupling matrix.

Although some of the derivations are somewhat involved, the final results are relatively simple. The additional mixing terms in the local damping and diffusion coefficients are more complicated than one might hope for, involving all the lattice functions, including dispersion and phase advances. However, as we have discussed, in typical situations, these terms contribute little to the global diffusion and damping, making the final results particularly simple; one simply needs to compute the global coupling angle $\theta$ for each resonance to find out how the uncoupled transverse and longitudinal (or horizontal and vertical for $x-y$ coupling) global diffusion and damping coefficients and emittances mix together.

Because of the relative simplicity of the final analytic expressions for equilibrium invariants and resonance widths, understanding of how these important properties depend on machine parameters is facilitated in a way which would sometimes be more convenient than purely numerical calculations.

\section{ACKNOWLEDGMENTS}

The authors would like to thank Dr. Etienne Forest, Dr. Georg Hoffstaetter, and Dr. Katsunobu Oide for their interest and comments. One of us (B.N.) would like to thank Ben Freivogel for useful discussions of this material. This work was supported by the U.S. Department of Energy under Contract No. DE-AC0376 SF00515.

\section{APPENDIX A: INVARIANTS AND SECOND- MOMENT MATRIX}

We would like to show that the expressions given for the invariants $G_{a}$ in the text, do in fact satisfy the invariant condition. That is, we would like to show that

$$
G_{a}=J U H_{a} U^{T} J
$$

satisfies

$$
M^{T} G_{a} M=G_{a} .
$$

Using the symplectic property of $M$, and Eq. (22), we derive

$$
\begin{aligned}
M^{T} G_{a} M & =M^{T} J U H_{a} U^{T} J M=J M^{-1} U H_{a} U^{T} M^{T-1} J \\
& =J U e^{-\Lambda} H_{a} e^{-\Lambda} U^{T} J=J U H_{a} U^{T} J .
\end{aligned}
$$

In the last step we have done the matrix multiplication

$$
\left(\begin{array}{cc}
e^{-i \mu} & 0 \\
0 & e^{i \mu}
\end{array}\right)\left(\begin{array}{ll}
0 & 1 \\
1 & 0
\end{array}\right)\left(\begin{array}{cc}
e^{-i \mu} & 0 \\
0 & e^{i \mu}
\end{array}\right)=\left(\begin{array}{ll}
0 & 1 \\
1 & 0
\end{array}\right) .
$$

This shows that the $G_{a}$ given are in fact invariants.

Now we consider the beam distribution written in terms of the invariants. We write a general (6D) Gaussian distribution as

$$
f(\vec{z})=\frac{1}{(2 \pi)^{3} \sqrt{\operatorname{det}\left(\mathbb{M}^{-1}\right)}} e^{-(1 / 2) \vec{z}^{T} M \vec{z}}
$$

where we have normalized $f$ so that $\int f(\vec{z}) d \vec{z}=1$. By performing the integration, one can show that the secondmoment matrix is given by

$$
\Sigma_{j k}=\left\langle z_{j} z_{k}\right\rangle=\mathbb{M}_{j k}^{-1} .
$$

In terms of the three invariants, we can write the distribution as

$$
f(\vec{z})=\frac{1}{\pi^{3}\left\langle g_{1}\right\rangle\left\langle g_{2}\right\rangle\left\langle g_{3}\right\rangle} \exp \left(-\frac{g_{1}}{\left\langle g_{1}\right\rangle}-\frac{g_{2}}{\left\langle g_{2}\right\rangle}-\frac{g_{3}}{\left\langle g_{3}\right\rangle}\right) .
$$

Let us prove this. In particular, we must show that the quantities we have written as $\left\langle g_{a}\right\rangle$ in the above equation are indeed given by

$$
\left\langle g_{a}\right\rangle=\int d \vec{z} g_{a} f(\vec{z})
$$

To show this, we introduce the matrix $K$ given by

$$
K=\frac{1}{\sqrt{2}}\left(\begin{array}{cccccc}
1 & -i & 0 & 0 & 0 & 0 \\
-i & 1 & 0 & 0 & 0 & 0 \\
0 & 0 & 1 & -i & 0 & 0 \\
0 & 0 & -i & 1 & 0 & 0 \\
0 & 0 & 0 & 0 & 1 & -i \\
0 & 0 & 0 & 0 & -i & 1
\end{array}\right)
$$

Note that $\operatorname{det}(K)=1$. In fact, it is symplectic. Now, consider the change of coordinates from $\vec{z}$ to the coordinates $\vec{V}$ 


$$
\vec{z}=U K \vec{V}
$$

$U K$ is in fact a real symplectic matrix. Its columns are $\sqrt{2}$ times the real and imaginary parts of $v_{a}$. The quadratic form $g_{a}$ becomes

$$
\begin{aligned}
g_{a} & =\vec{z}^{T} G_{a} \vec{z}=\vec{V}^{T} K^{T} U^{T} G_{a} U K \vec{V}=\vec{V}^{T} K^{T} H_{a} K \vec{V} \\
& =\vec{V}^{T} I_{a} \vec{V} .
\end{aligned}
$$

Where $I_{a}$ is a matrix that is all zeros except for a $2 \times 2$ identity matrix in the $a$ th diagonal $2 \times 2$ block. Thus, $g_{a}=$ $V_{a}^{2}+V_{-a}^{2}$ (with $a=1,2,3$ for $g_{a}$, and $k= \pm 1, \pm 2, \pm 3$ for $V_{k}$ ). Applying this change of variables to (A8), we get

$$
\int d \vec{V} \frac{V_{a}^{2}+V_{-a}^{2}}{\pi^{3}\left\langle g_{1}\right\rangle\left\langle g_{2}\right\rangle\left\langle g_{3}\right\rangle} e^{-\frac{V_{1}^{2}+V_{-1}^{2}}{\left\langle g_{1}\right\rangle}-\frac{V_{2}^{2}+V_{-2}^{2}}{\left\langle g_{2}\right\rangle}-\frac{V_{3}^{2}+V_{-3}^{2}}{\left\langle g_{3}\right\rangle}}=\left\langle g_{a}\right\rangle
$$

after doing the $61 \mathrm{D}$ integrals.

Next we find expressions for the second-moment matrix. Looking at (A5) and (A7), we identify

$$
\mathbb{M}=\frac{2 G_{1}}{\left\langle g_{1}\right\rangle}+\frac{2 G_{2}}{\left\langle g_{2}\right\rangle}+\frac{2 G_{3}}{\left\langle g_{3}\right\rangle} .
$$

Thus, to find the second-moment matrix $\Sigma$, we must invert this matrix. We claim that the inverse is given by

$$
\mathbb{M}^{-1}=-\frac{1}{2}\left\langle g_{1}\right\rangle J G_{1} J-\frac{1}{2}\left\langle g_{2}\right\rangle J G_{2} J-\frac{1}{2}\left\langle g_{3}\right\rangle J G_{3} J .
$$

In order to prove this, we show the following:

$$
J G_{a} J G_{b}=-\delta_{a b} I_{a},
$$

where $a$ and $b$ run from 1 to 3 . Now, $G_{a}$ is written explicitly as

$$
G_{a}=J U H_{a} U^{T} J .
$$

Substituting this in and using the symplectic property of $U$ and the fact that $J^{2}=-I$, we get

$$
J G_{a} J G_{b}=U H_{a} J H_{b} J U^{-1} .
$$

From this and the definition of $H_{a}$, (25), Eq. (A19) follows by simple matrix multiplication. In particular, when $a=b$, we find

$$
\sigma_{x} J_{2} \sigma_{x} J_{2}=\left(\begin{array}{ll}
1 & 0 \\
0 & 1
\end{array}\right)
$$

The minus sign comes from the two factors of $i$ from the $H_{a}$. Now, multiplication of (A14) by (A15) and repeated use of Eq. (A16), proves that (A15) is indeed the inverse matrix.

\section{APPENDIX B: GENERAL 2ND ORDER DEGENERATE PERTURBATION THEORY}

Let us suppose we have a matrix $M$ that can be expanded as

$$
M=\sum_{p} M_{p}
$$

where we consider $M_{p}$ to be of order $\epsilon^{p}$ where $\epsilon$ is some small parameter. We seek a perturbation expansion of the eigenvectors and eigenvalues of $M$ :

$$
M v_{k}=\lambda_{k} v_{k}
$$

with

$$
\boldsymbol{v}_{k}=\sum_{p} c_{k 0}^{p} \tilde{\boldsymbol{v}}_{k p}
$$

and

$$
\lambda_{k}=\sum_{p} \lambda_{k p}
$$

We consider $\tilde{\boldsymbol{v}}_{k p}$ and $\lambda_{k p}$ to be of order $\epsilon^{p}$. We use the tilde in $\tilde{\boldsymbol{v}}_{k p}$ because we would like to distinguish $\tilde{\boldsymbol{v}}_{k 0}$ from $\boldsymbol{v}_{k 0}$. Let $Z_{d g}(k)$ be the indices such that for $j \in Z_{d g}(k), \lambda_{j 0}=$ $\lambda_{k 0}$. In other words, $Z_{d g}(k)$ is the set of indices with eigenvalues that are degenerate with $\lambda_{k 0}$. We let $\left\{\boldsymbol{v}_{k 0}\right\}$ be an orthonormal complete set of eigenvectors of $M_{0}$. In particular, we assume that we can define covectors $v^{l 0}$ such that

$$
v^{l 0} v_{k 0}=\delta_{l k}
$$

How to do this for a symplectic matrix is discussed in the main text. This means that we can expand an arbitrary vector as

$$
v=\sum_{k} c_{k} v_{k 0}
$$

with

$$
c_{k}=v^{k 0} v .
$$

We consider the $v_{k 0}$ to be the "uncoupled," "unperturbed" eigenvectors. Within the perturbation matrices $M_{1}$ and $M_{2}$, we consider there as being two different types of perturbations. In the case that we consider them both as 1st order (an important case will occur when we consider it as 2nd order), we notate them as $M_{1 \xi}$ and $M_{1 \delta \mu}$. When just $M_{1 \delta \mu}$ is added to $M_{0}$, its effect is to change the eigenvalues, but not the $\boldsymbol{v}_{k 0}$. This is in effect how we define $\boldsymbol{v}_{k 0}$. The addition of the perturbation $M_{1 \xi}$ serves to pick out a particular linear combination of the $v_{k 0}$ which we notate as $\tilde{v}_{k 0}$, in addition to causing a perturbation of these eigenvectors. However, it will be the main purpose of this section to derive the $\tilde{\boldsymbol{v}}_{k 0}$ for the two cases where we have a $M_{1 \delta \mu}$ and a $M_{2 \delta \mu}$, i.e., when the purely eigenvalue changing perturbation is first order and second order. It will turn out that this latter case is important in the case of "coupling" causing an integer or half-integer resonance. 
We now consider the eigenvalue equation setting equal terms of the same order up to 2 nd order:

$$
\begin{aligned}
p & =0: M_{0} \tilde{\boldsymbol{v}}_{k 0}=\lambda_{k 0} \tilde{\boldsymbol{v}}_{k 0}, \\
p & =1: M_{0} \tilde{\boldsymbol{v}}_{k 1}+M_{1} \tilde{\boldsymbol{v}}_{k 0}=\lambda_{k 0} \tilde{\boldsymbol{v}}_{k 1}+\lambda_{k 1} \tilde{\boldsymbol{v}}_{k 0} \\
p & =2: M_{0} \tilde{\boldsymbol{v}}_{k 2}+M_{1} \tilde{\boldsymbol{v}}_{k 1}+M_{2} \tilde{\boldsymbol{v}}_{k 0} \\
& =\lambda_{k 0} \tilde{\boldsymbol{v}}_{k 2}+\lambda_{k 1} \tilde{\boldsymbol{v}}_{k 1}+\lambda_{k 0} \tilde{\boldsymbol{v}}_{k 2} .
\end{aligned}
$$

Consider the $p=0$ equation. Expanding out the $\tilde{v}_{k 0}$ and using the 0 th order eigenvector equation, we get

$$
\sum_{j} c_{k 0}^{j} \lambda_{j 0} v_{j 0}=\lambda_{k 0} \sum_{j} c_{k 0}^{j} v_{j 0} .
$$

Multiplying on the left by $v^{l 0}$ gives

$$
c_{k 0}^{l} \lambda_{l 0}=c_{k 0}^{l} \lambda_{k 0}
$$

which implies that either $\lambda_{l 0}=\lambda_{k 0}$ or $c_{k 0}^{l}=0$. This is what we expect since for $l \in Z_{d g}(k), \lambda_{l 0}=\lambda_{k 0}$ and otherwise $c_{k 0}^{l}=0$.

Next we consider $p=1$. Expanding the $\tilde{v}_{k}$ and again using the 0th order eigenvector equation gives

$$
\sum_{j} c_{k 1}^{j} \lambda_{j 0} v_{j 0}+M_{1} \sum_{j} c_{k 0}^{j} v_{j 0}=\lambda_{k 0} \sum_{j} c_{k 1}^{j} v_{j 0}+\lambda_{k 1} \sum_{j} c_{k 0}^{j} v_{j 0} .
$$

We now multiply on the left by $v^{l 0}$. The result is

$$
c_{k 1}^{l} \lambda_{l 0}+\sum_{j} c_{k 0}^{j} \mathcal{M}_{l j}=\lambda_{k 0} c_{k 1}^{l}+\lambda_{k 1} c_{k 0}^{l},
$$

where we have defined

$$
\mathcal{M}_{l j}=v^{l 0} M_{1} v_{j 0} .
$$

Now, suppose that $l \in Z_{d g}(k)$. Then $\lambda_{l 0}=\lambda_{k 0}$ and two of the terms cancel. We are left with

$$
\sum_{j} c_{k 0}^{j} \mathcal{M}_{l j}=\lambda_{k 1} c_{k 0}^{l}, \quad l \in Z_{d g}(k) .
$$

We will use this equation to analyze the sum and difference resonances. In the case where there is no degeneracy, $Z_{d g}(k)=\{k\}$ and we get the usual result from 1st order nondegenerate perturbation theory:

$$
\lambda_{k 1}=\mathcal{M}_{k k} \text {. }
$$

Next suppose that $l \notin Z_{d g}(k)$. Then $\lambda_{l 0} \neq \lambda_{k 0}$ and $c_{k 0}^{l}=0$. Then Eq. (B12) gives

$$
c_{k 1}^{l}=\frac{\sum_{j} c_{k 0}^{j} \mathcal{M}_{l j}}{\lambda_{k 0}-\lambda_{l 0}}, \quad l \notin Z_{d g}(k) .
$$

Finally, we consider $p=2$. Expanding out the $\tilde{\boldsymbol{v}}_{k p}$, using the $M_{0}$ eigenvalue equation and left multiplying by $v^{l 0}$ gives

$$
\begin{gathered}
c_{k 2}^{l} \lambda_{l 0}+\sum_{j} c_{k 1}^{j} \mathcal{M}_{l j}+\sum_{j} c_{k 0}^{j} \mathcal{M}_{2, l j} \\
=\lambda_{k 0} c_{k 2}^{l}+\lambda_{k 1} c_{k 1}^{l}+\lambda_{k 2} c_{k 0}^{l},
\end{gathered}
$$

where we have defined

$$
\mathcal{M}_{2, l j} \equiv v^{l 0} M_{2} v_{j 0} .
$$

Now, suppose that $l \in Z_{d g}(k)$. Then $\lambda_{l 0}=\lambda_{k 0}$. We can then write

$$
c_{k 0}^{l} \lambda_{k 2}=\sum_{j} c_{k 1}^{j} \mathcal{M}_{l j}+\sum_{j} c_{k 0}^{j} \mathcal{M}_{2, l j}-\lambda_{k 1} c_{k 1}^{l}, \quad l \in Z_{d g}(k) .
$$

There are two different contexts in which one might use this equation. The first is that the $c_{k 0}^{l}$ have already been determined by the lower order equations and this becomes an equation for just $\lambda_{k 2}$. This is the case if $M_{1}$ breaks the degeneracy. In this case $\lambda_{k 1}$ is nonzero. We then think of this equation as adding small terms to the eigenvectors and eigenvalues. Another important possibility is where $M_{1}$ does not break the degeneracy. This happens when all the $\mathcal{M}_{l k}$ 's are zero for $l \in Z_{d g}(k)$. This means that $\lambda_{k 1}=0$. Then, after using Eqs. (B13) and (B19) becomes an eigenvalue equation for $c_{k 0}^{l}$ and $\lambda_{k 2}$ :

$$
\sum_{j}\left[\sum_{n \notin Z_{d g}(k)} \frac{\mathcal{M}_{n j} \mathcal{M}_{l n}}{\lambda_{k 0}-\lambda_{n 0}}+\mathcal{M}_{2, l j}\right] c_{k 0}^{j}=\lambda_{k 2} c_{k 0}^{l}, \quad l \in Z_{d g}(k) .
$$

This is our main result which we use to analyze the integer and half-integer resonances caused by coupling.

If we take the case where there is no degeneracy, this reduces to

$$
\lambda_{k 2}=\sum_{n \neq k} \frac{\mathcal{M}_{n k} \mathcal{M}_{k n}}{\lambda_{k 0}-\lambda_{n 0}}+\mathcal{M}_{2, k k}
$$

which, if we also set $\mathcal{M}_{2, k k}=0$ gives the usual expression for the second order shift in the eigenvalue for nondegenerate perturbation theory.

Now, let $l \notin Z_{d g}(k)$. Then, as before, $\lambda_{l 0} \neq \lambda_{k 0}$ and $c_{k 0}^{l}=0$. Solving for $c_{k 2}^{l}$ gives

$$
c_{k 2}^{l}=\frac{\sum_{j} c_{k 1}^{j} \mathcal{M}_{l j}+\sum_{j} c_{k 0}^{j} \mathcal{M}_{2, l j}-\lambda_{k 1} c_{k 1}^{l}}{\lambda_{k 0}-\lambda_{l 0}}, \quad l \notin Z_{d g}(k) .
$$

\section{APPENDIX C: RELATIONSHIP BETWEEN $\xi_{+}$AND $\xi_{-}$}

Suppose we have a general matrix $Q$ with elements

$$
Q=\left(\begin{array}{ll}
q_{11} & q_{12} \\
q_{21} & q_{22}
\end{array}\right)
$$


Now, compute the quantities

$$
\begin{aligned}
\frac{1}{2}\left(\begin{array}{ll}
i & 1
\end{array}\right) Q\left(\begin{array}{l}
1 \\
i
\end{array}\right) & =\frac{1}{2}\left[\left(q_{21}-q_{12}\right)+i\left(q_{11}+q_{22}\right)\right], \\
\frac{1}{2}\left(\begin{array}{ll}
-i & 1
\end{array}\right) Q\left(\begin{array}{l}
1 \\
i
\end{array}\right) & =\frac{1}{2}\left[\left(q_{21}+q_{12}\right)+i\left(-q_{11}+q_{22}\right)\right] .
\end{aligned}
$$

Now, defining

$$
\mathcal{A}_{x, z}=\left(\begin{array}{cc}
\sqrt{\beta_{x, z}} & 0 \\
\frac{-\alpha_{x, z}}{\sqrt{\beta_{x, z}}} & \frac{1}{\sqrt{\beta_{x, z}}}
\end{array}\right),
$$

and identifying

$$
Q=\mathcal{A}_{x}^{T} J B \mathcal{A}_{z}
$$

where $B$ is the upper right $2 \times 2$ block of $M_{1}$ or $P$, we can see that

$$
\left|\mathcal{M}_{12}\right|^{2}-\left|\mathcal{M}_{-12}\right|^{2}=\operatorname{det}(Q) .
$$

But, since $\operatorname{det}\left(\mathcal{A}_{x, z}\right)=\operatorname{det}(J)=1$, we get that $\operatorname{det}(Q)=$ $\operatorname{det}(B)$, and hence

$$
\xi_{-}^{2}-\xi_{+}^{2}=4 \operatorname{det}(B)
$$

\section{APPENDIX D: FURTHER DERIVATIONS FOR THE COUPLING INTEGER/HALF-INTEGER RESONANCES}

Suppose that the transfer matrix $M$ is close to the $\mu_{z}$ integer resonance. Then the eigenvalue equation can be written in the form [22]

$$
\left(\begin{array}{cc}
M_{x} & B \\
C & I+D
\end{array}\right)\left(\begin{array}{l}
u \\
v
\end{array}\right)=(1+\delta \lambda)\left(\begin{array}{l}
u \\
v
\end{array}\right)
$$

where we are looking for the eigenvalues near 1. $u$ and $v$ are $1 \times 2$ column matrices, and we assume that $B, C, D$ and $\delta \lambda$ are small. If the upper equation is solved for $u$, we find

$$
u=-\left[M_{x}-I(1+\delta \lambda)\right]^{-1} v .
$$

Substituting this into the lower equation and taking lowest order in the small quantities (i.e., ignoring $\delta \lambda$ in above equation), we get

$$
\left[D-C\left(M_{x}-I\right)^{-1} B\right] v=\delta \lambda v .
$$

Thus, we must find eigenvalues and eigenvectors of this $2 \times 2$ matrix. If we change into the basis in which $M$ is diagonal, and taking into account the half-integer resonance as well, in which $I \rightarrow-I$, we get

$$
\left(\begin{array}{cc}
\frac{\mathcal{M}_{12} \mathcal{M}_{21}}{\lambda_{20}-\lambda_{10}} & \frac{\mathcal{M}_{1-2} \mathcal{M}_{21}}{\lambda_{20}-\lambda_{10}} \\
+\frac{\mathcal{M}_{-12} \mathcal{M}_{2-1}}{\lambda_{20}-\lambda_{-10}} & +\frac{\mathcal{M}_{-1-2} \mathcal{M}_{2-1}}{\lambda_{20}-\lambda_{-10}} \\
+\mathcal{M}_{22} & +\mathcal{M}_{2-2} \\
\frac{\mathcal{M}_{12} \mathcal{M}_{-21}}{\lambda_{20}-\lambda_{10}} & \frac{\mathcal{M}_{1-2} \mathcal{M}_{-21}}{\lambda_{20}-\lambda_{10}} \\
+\frac{\mathcal{M}_{-12} \mathcal{M}_{-2-1}}{\lambda_{20}-\lambda_{-10}} & +\frac{\mathcal{M}_{-1-2} \mathcal{M}_{-2-1}}{\lambda_{20}-\lambda_{-10}} \\
+\mathcal{M}_{-22} & +\mathcal{M}_{-2-2}
\end{array}\right) .
$$

For the integer resonance, this simplifies to

$$
\left(\begin{array}{cc}
\frac{\left|r_{12}\right|^{2}}{1-e^{-i \mu_{x}}}-\frac{\left|r_{-12}\right|^{2}}{1-e^{i \mu_{x}}} & i r_{21} r_{1-2} \cot \left(\frac{\mu_{x}}{2}\right)+r_{2-2} \\
+r_{22} & \frac{\left|r_{12}\right|^{2}}{1-e^{i \mu_{x}}}-\frac{\left|r_{-12}\right|^{2}}{1-e^{-i \mu_{x}}} \\
-i r_{21}^{*} r_{1-2}^{*} \cot \left(\frac{\mu_{x}}{2}\right)+r_{-22} & +r_{-2-2}
\end{array}\right)
$$

for the half integer it is

$$
\left(\begin{array}{cc}
\frac{-\left|r_{12}\right|^{2}}{1+e^{-i \mu_{x}}}+\frac{\left|r_{-12}\right|^{2}}{1+e^{i \mu_{x}}} & i r_{21} r_{1-2} \tan \left(\frac{\mu_{x}}{2}\right)-r_{2-2} \\
-r_{22} & \frac{-\left|r_{12}\right|^{2}}{1+e^{i \mu_{x}}}+\frac{\left|r_{-12}\right|^{2}}{1+e^{-i \mu_{x}}} \\
-i r_{21}^{*} r_{1-2}^{*} \tan \left(\frac{\mu_{x}}{2}\right)-r_{-22} & -r_{-2-2}
\end{array}\right) .
$$

Now, for a matrix,

$$
\left(\begin{array}{ll}
a & b \\
c & d
\end{array}\right)
$$

the eigenvalues can be written as

$$
\delta \lambda_{ \pm}=\frac{1}{2}\left[a+d \pm(a-d) \sqrt{1+\frac{4 b c}{(a-d)^{2}}}\right]
$$

and the (unnormalized) eigenvectors as

$$
v_{ \pm}=\left(\frac{1}{2 c}\left[(a-d) \pm(a-d) \sqrt{1+\frac{4 b c}{(a-d)^{2}}}\right]\right) .
$$

In order to be consistent with the notation for the simpler resonances, we make the following definitions

$$
\begin{gathered}
\Delta \mu=-i e^{-i \mu_{0}}(a-d), \\
\xi=2|b|,
\end{gathered}
$$

and

$$
\phi=\arg \left(e^{i \mu_{0}} b\right)
$$

where $\mu_{0}$ is 0 for the integer resonance and $\pi$ for the halfinteger. This phase definition being consistent depends on the fact that $c^{*}=b$ which is easily verified for each of the two cases above. The perturbed phase advance is given by

$$
\mu_{2}=\frac{\Delta \mu}{2} \sqrt{1-\frac{\xi^{2}}{\Delta \mu^{2}}}
$$

for the integer resonance and 


$$
\mu_{2}=\pi+\Delta \mu^{2} \sqrt{1-\frac{\xi^{2}}{\Delta \mu^{2}}}
$$

for the half-integer resonance. The perturbed eigenvector is given by

$$
v_{2}=\cosh \left(\frac{\theta}{2}\right) v_{z}-i e^{-i \phi} \sinh \left(\frac{\theta}{2}\right) v_{-z}
$$

with

$$
\tanh (\theta)=\frac{\xi}{\Delta \mu}
$$

Writing out all of these quantities explicitly, and after some algebra, we find for the integer resonance

$$
\begin{gathered}
\Delta \mu=2 \mu_{z}-2 i r_{22 \xi}-\left(\left|r_{12}\right|^{2}+\left|r_{-12}\right|^{2}\right) \cot \left(\frac{\mu_{x}}{2}\right), \\
\quad \xi=2\left|r_{2-2}+i r_{1-2} r_{21} \cot \left(\frac{\mu_{x}}{2}\right)\right|,
\end{gathered}
$$

and

$$
\phi=\arg \left[r_{2-2}+i r_{1-2} r_{21} \cot \left(\frac{\mu_{x}}{2}\right)\right]
$$

For the half-integer we get

$$
\begin{array}{r}
\Delta \mu=2\left(\mu_{z}-\pi\right)-2 i r_{22 \xi}+\left(\left|r_{12}\right|^{2}+\left|r_{-12}\right|^{2}\right) \tan \left(\frac{\mu_{x}}{2}\right), \\
\quad \xi=2\left|r_{2-2}-i r_{1-2} r_{21} \tan \left(\frac{\mu_{x}}{2}\right)\right|, \quad \text { (D20) }
\end{array}
$$

and

$$
\phi=\arg \left[r_{2-2}-i r_{1-2} r_{21} \tan \left(\frac{\mu_{x}}{2}\right)\right]
$$

In each case, we can combine $\xi$ and $\Delta \mu$ to find the perturbed value of $\mu_{2}$ using (D13) and (D14). We ignore the issue of the sign here and compute $\mu_{2}^{2}$ :

$$
\begin{aligned}
\mu_{2}^{2}= & \left(\mu_{z}-i r_{22 \xi}\right)^{2}-\left(\mu_{z}-i r_{22 \xi}\right)\left(\left|r_{12}\right|^{2}+\left|r_{1-2}\right|^{2}\right) \\
& \times \cot \left(\frac{\mu_{x}}{2}\right)-\left|r_{2-2 \xi}\right|^{2}+\frac{1}{4}\left(\left|r_{12}\right|^{2}-\left|r_{1-2}\right|^{2}\right) \cot ^{2}\left(\frac{\mu_{x}}{2}\right) \\
& \left.+\frac{1}{2} \operatorname{Im}\left(r_{2-2 \xi}^{*} r_{12} r_{1-2}\right) \cot \left(\frac{\mu_{x}}{2}\right) \quad \text { (int } z\right), \quad \text { (D23) }
\end{aligned}
$$

$$
\begin{aligned}
\left(\mu_{2}-\pi\right)^{2}= & {\left[\left(\mu_{z}-\pi\right)-i r_{22 \xi}\right]^{2}+\left(\left(\mu_{z}-\pi\right)-i r_{22 \xi}\right) } \\
& \times\left(\left|r_{12}\right|^{2}+\left|r_{1-2}\right|^{2}\right) \tan \left(\frac{\mu_{x}}{2}\right)-\left|r_{2-2 \xi}\right|^{2}+\frac{1}{4} \\
& \times\left(\left|r_{12}\right|^{2}-\left|r_{1-2}\right|^{2}\right) \tan ^{2}\left(\frac{\mu_{x}}{2}\right) \\
& -\frac{1}{2} \operatorname{Im}\left(r_{2-2 \xi}^{*} r_{12} r_{1-2}\right) \tan \left(\frac{\mu_{x}}{2}\right) \quad\left(\frac{1}{2} \text { int } z\right) .
\end{aligned}
$$

Note that for both cases $\mu_{2}$ reduces to $\mu_{z}$ when the perturbation is turned off.

Now, we consider a useful special case. Suppose that the perturbation $M_{1 \xi}$ only has off-block diagonal elements. This implies that $r_{22 \xi}=r_{2-2 \xi}=0$. Further suppose that $\left|r_{12}\right|^{2}=\left|r_{-12}\right|^{2}$. This is equivalent to $\operatorname{det}(B)=0$, where $B$ is the off-block diagonal matrix in either $M_{1 \xi}$ or $P$. If these two conditions hold, the expressions for $\mu_{2}$ simplify considerably. They become

$$
\begin{gathered}
\mu_{2}^{2}=\mu_{z}^{2}-2 \mu_{z}\left|r_{12}\right|^{2} \cot \left(\frac{\mu_{x}}{2}\right), \\
\left(\mu_{2}-\pi\right)^{2}=\left(\mu_{z}-\pi\right)^{2}+2\left(\mu_{z}-\pi\right)\left|r_{12}\right|^{2} \tan \left(\frac{\mu_{x}}{2}\right)
\end{gathered}
$$

for the integer and half-integer, respectively.

The expressions for $x$ are given by interchanging 1 and 2 , and $x$ and $z$.

Finally, consider the quantity $\bar{\mu}$ which we expect to be 0 for the integer and half-integer resonances. It is proportional to the trace of (D4). Computing this quantity, we find that it is not zero. A careful analysis, however, reveals that it is always higher order than the lowest order terms kept and can thus be seen as a part of the higher order terms in the expansion of $e^{i \mu}$ which should be dropped.

[1] M. Sands, SLAC Report No. SLAC-121, 1970 (unpublished).

[2] SLAC Conceptual Design Report No. SLAC-418, 1993.

[3] KEKB B-Factory Design Report No. 95-7, 1995.

[4] E. D. Courant and H. S. Snyder, Ann. Phys. (N.Y.) 3, 1 (1958).

[5] G. H. Hoffstaetter and A. W. Chao, Phys. Rev. ST Accel. Beams 7, 071002 (2004).

[6] B. Nash, J. Wu, K. Bane, and A. Chao, in Proceedings of the Particle Accelerator Conference, Portland, OR, 2003 (IEEE, Piscataway, NJ, 2003).

[7] K. Kubo and K. Oide, Phys. Rev. ST Accel. Beams 4, 124401 (2001).

[8] R. H. Helm, M. J. Lee, P. L. Morton, and M. Sands, IEEE Trans. Nucl. Sci. 20, 900 (1973).

[9] A. Chao, J. Appl. Phys. 50, 595 (1979). 
[10] F. Ruggiero, E. Picasso, and L. Radicati, Ann. Phys. (N.Y.) 197, 396 (1990).

[11] K. Ohmi, K. Hirata, and K. Oide, Phys. Rev. E 49, 751 (1994).

[12] D. P. Barber, K. Heinemann, H. Mais, and G. Ripken, DESY-91-146, 1991.

[13] E. Forest (private communication).

[14] Y. Cai (private communication).

[15] J. Wu, B. Nash, and A. W. Chao, in Proceedings of the Particle Accelerator Conference, Knoxville, TN, 2005 (IEEE, Piscataway, NJ, 2005).

[16] D. A. Edwards and L. C. Teng, IEEE Trans. Nucl. Sci. 20, 885 (1973).
[17] E. Forest, M.F. Reusch, D. L. Bruhwiler, and A. Amiry, Part. Accel. 45, 65 (1994).

[18] K. W. Robinson, Phys. Rev. 111, 373 (1958).

[19] A. Piwinski, DESY 93-187, 1993 (unpublished); Handbook of Accelerator Physics and Engineering, edited by A. Chao and M. Tigner (World Scientific, Singapore, 2002), Sec. 2.3.4.

[20] David J. Griffiths, Introduction to Quantum Mechanics (Prentice Hall, New Jersey, 1994).

[21] R. Palmer, in Proceedings of the Snowmass DPF Summer Study 1988 (World Scientific, 1988), p. 613.

[22] B. Freivogel (private communication). 